UCRL-ID-121673

\title{
Guidelines for Conducting Impact Tests on Shipping Packages for Radioactive Material
}

\author{
Gerald C. Mok, Roger W. Carlson, \\ Stephen C. Lu, and Larry E. Fischer
}

September 1995

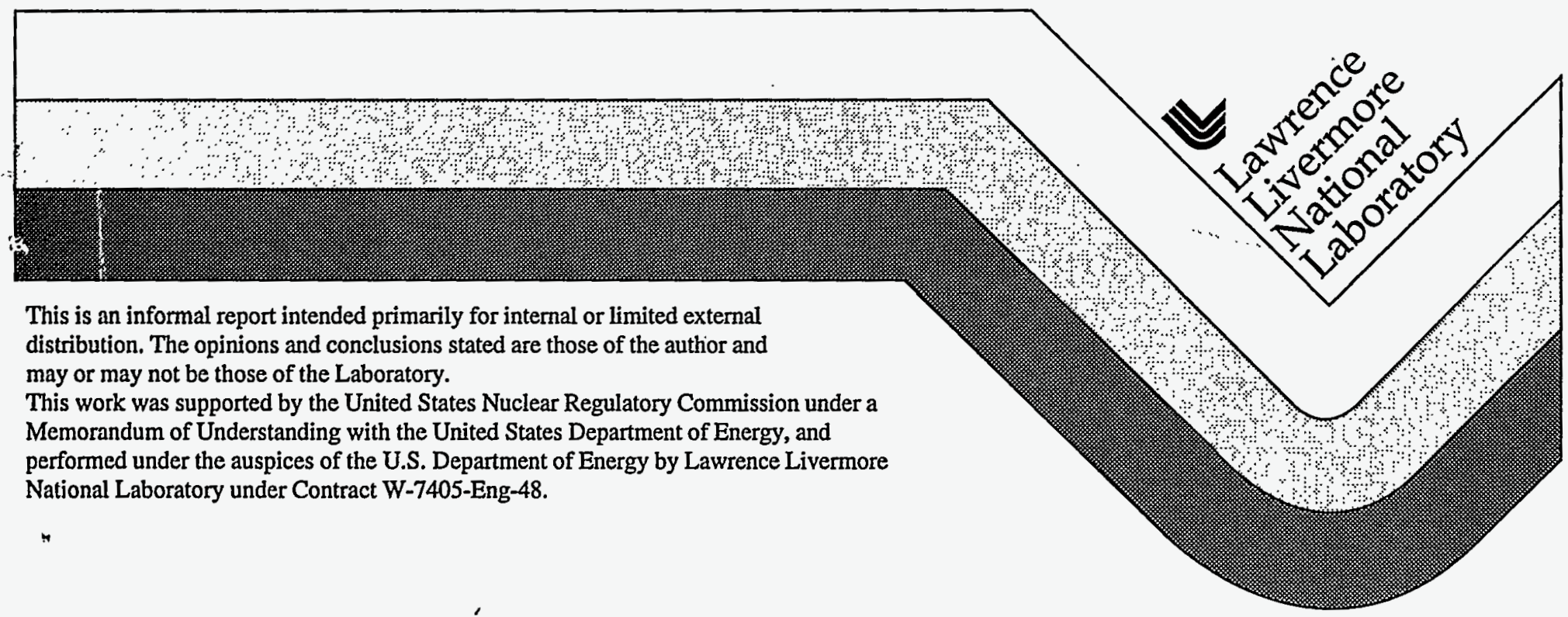

DISTRIBUTION OF THIS DOCUMENT IS UNLIMITED wW 


\section{Disclaimer}

This document was prepared as an account of work sponsored by an agency of the United States Government. Neither the United States Government nor the University of California nor any of their employees, makes any warranty, express or implied, or assumes any legal liability or responsibility for the accuracy, completeness, or usefulness of any information, apparatus, product, or process disclosed, or represents that its use would not infringe privately owned rights. Reference herein to any specific commercial product, process, or service by trade name, trademark, manufacturer, or otherwise, does not necessarily constitute or imply its endorsement, recommendation, or favoring by the United States Government or the University of California. The views and opinions of authors expressed herein do not necessarily state or reflect those of the United States Government or the University of California, and shall not be used for advertising or product endorsement purposes.

This report has been reproduced directly from the best available copy.

Available to DOE and DOE contractors from the

Office of Scientific and Technical Information

P.O. Box 62, Oak Ridge, TN 37831

Prices available from (615) 576-8401, FTS 626-8401

Available to the public from the

National Technical Information Service

U.S. Department of Commerce

5285 Port Royal Rd.,

Springfield, VA 22161 


\section{DISCLAIMIER}

Portions of this document may be illegible in electronic image products. Images are produced from the best available original document. 


\section{Guidelines for Conducting Impact Tests on Shipping Packages for Radioactive Material}

Prepared by:

Gerald C. Mok

Roger W. Carlson

Stephen C. Lu

Larry E. Fischer

Lawrence Livermore National Laboratory

7000 East Avenue

Livermore, California

Prepared for

U.S. Nuclear Regulatory Commission 
This page left blank intentionally. 


\begin{abstract}
Federal regulation (10 CFR Part 71) specifies a number of impact conditions (free-drop, penetration, and puncture), under which a package for the transport of radioactive materials must be tested or evaluated to demonstrate compliance with the regulation. This report is a comprehensive guide to the planning and execution of these impact tests. The report identifies the required considerations for both the design, pre-, and post-test inspections of the test model and the measurement, recording, analysis, and reporting of the test data. The report also presents reasons for the requirements, identifies the major difficulties in meeting these requirements, and suggests possible methods to overcome the difficulties. Discussed in substantial detail is the use of scale models and instrumented measurements.
\end{abstract}


This page left blank intentionally. 


\section{TABLE OF CONTENTS}

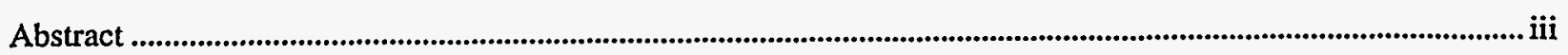

Executive Summary .........................................................................................................................................................

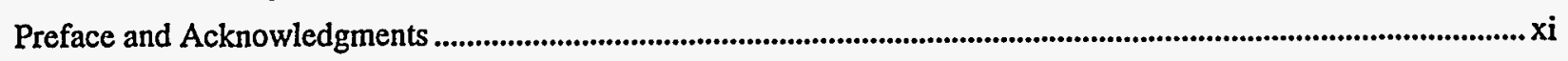

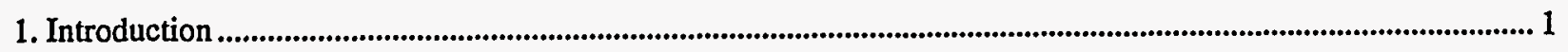

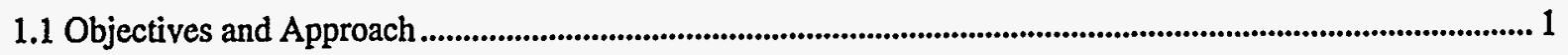

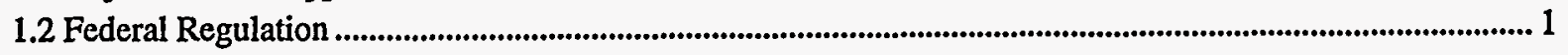

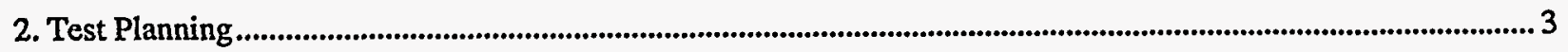

2.1 Basic Considerations ..........................................................................................................................................

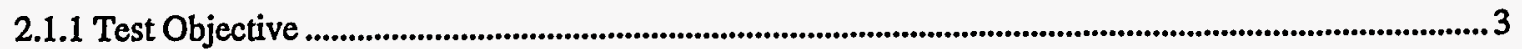

2.1.2 Impact Response and Package Design ........................................................................................................4

2.1.3 Structural Failure Modes and Material Properties ............................................................................................. 5

2.2 General Requirements ....................................................................................................................................

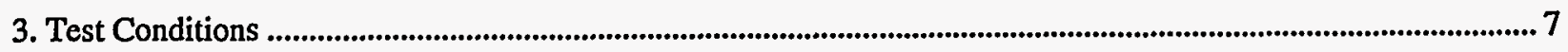

3.1 Basic Considerations .................................................................................................................................

3.2 Critical Test Conditions ....................................................................................................................................... 7

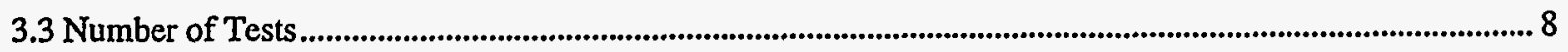

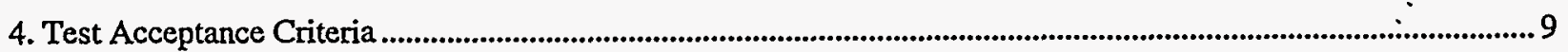

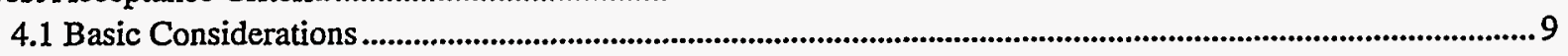

4.2 Procedures and Standards ..............................................................................................................................

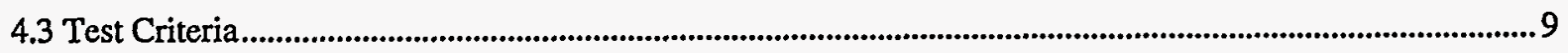

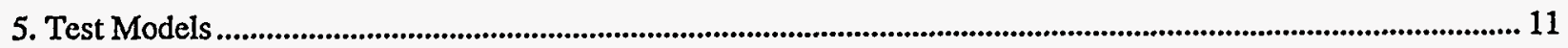

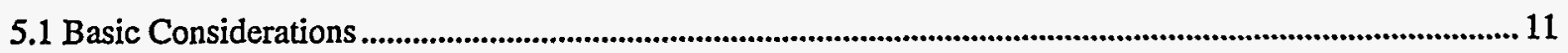

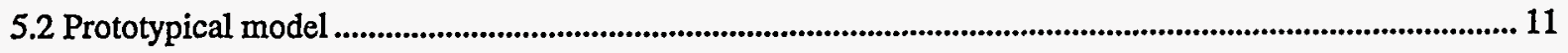

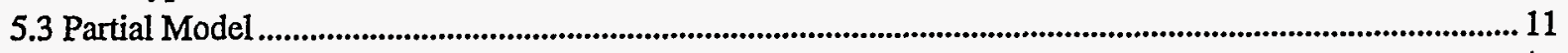

5.4 Simplified Model ................................................................................................................................................ 11

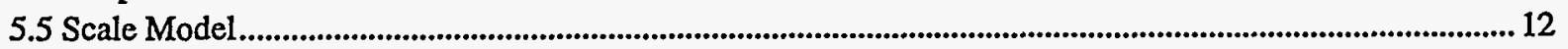

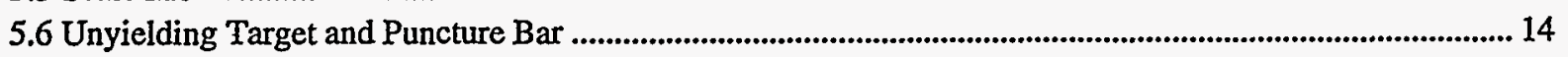

6. Pre- and Post-Test Inspections ............................................................................................................................... 17

6.1 Basic Considerations ........................................................................................................................................ 17

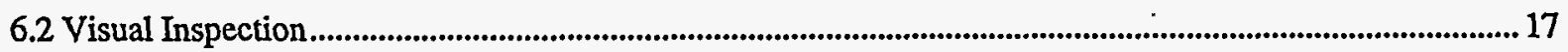

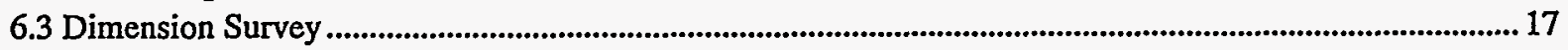

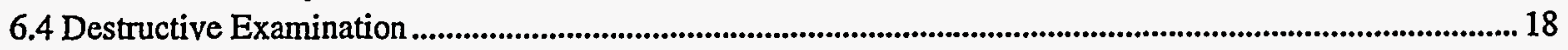

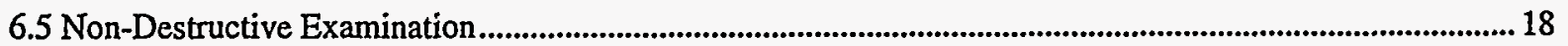

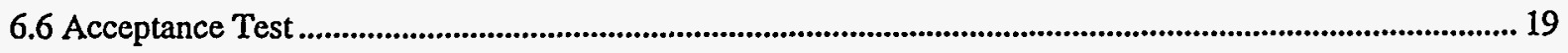

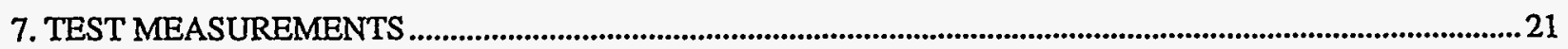

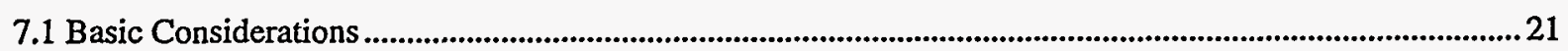

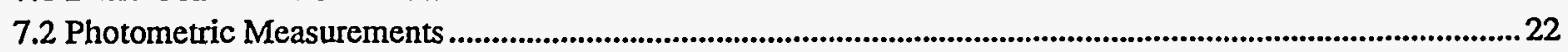

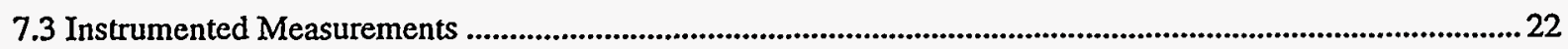

7.4 Other Measurements ..................................................................................................................................... 23

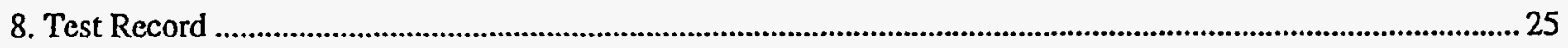

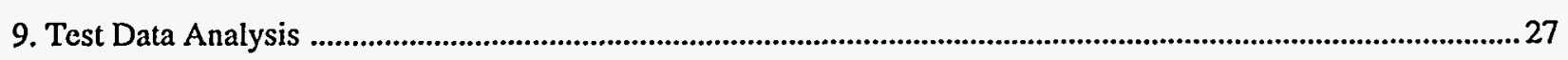

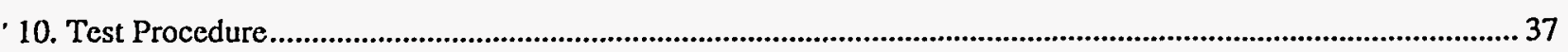

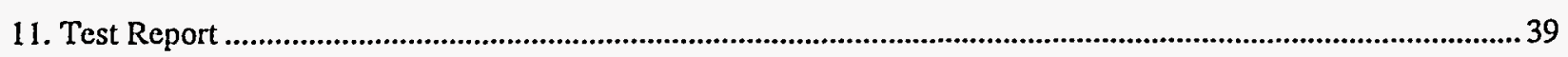

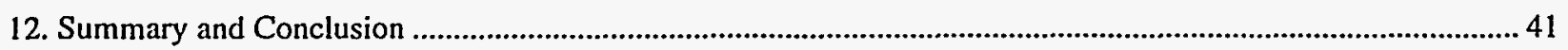




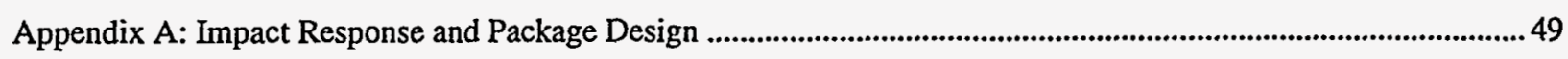

Appendix B: Scale-Model Impact Testing .................................................................................................................5

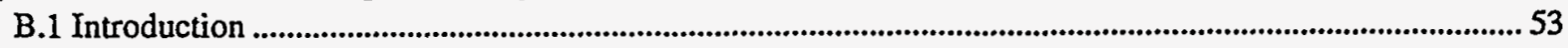

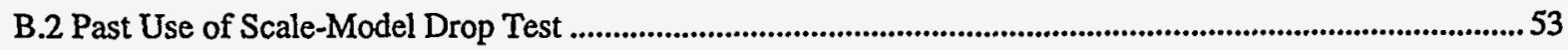

B.3 Scale Model and Scaling Laws .................................................................................................................. 53

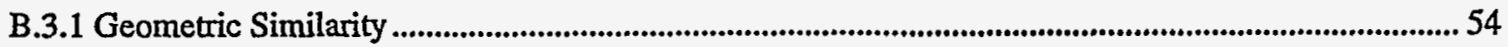

B.3.2 Kinematic Similarity ........................................................................................................................... 55

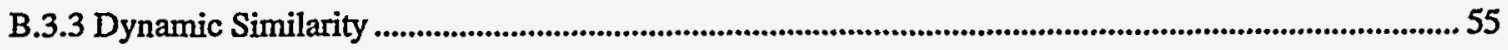

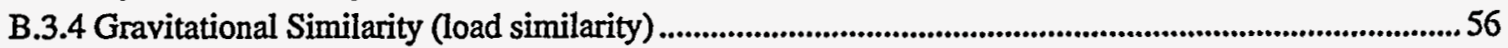

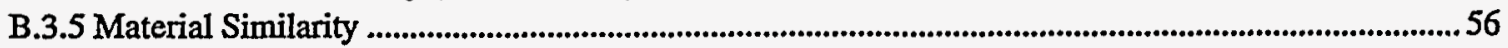

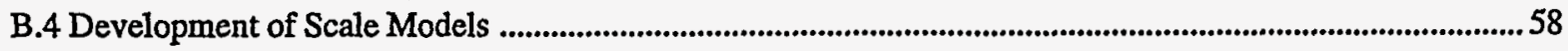

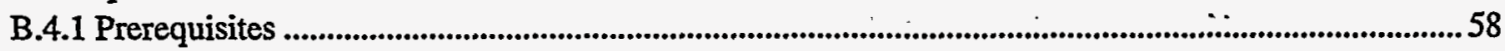

B.4.2 Valid Scale Models and Their Limitations …...............................................................................5 59

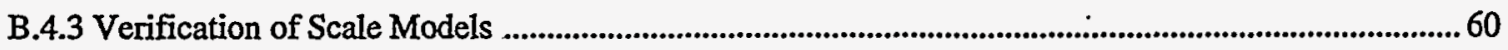

B.5 Other Considerations in Scale-Model Testing .....................................................................................61

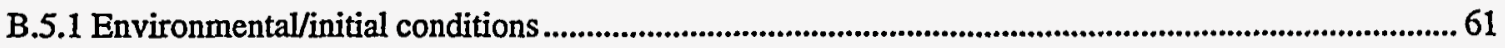

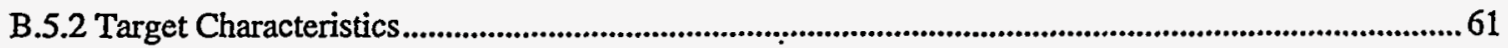

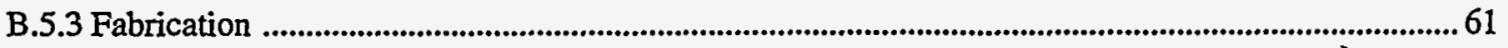

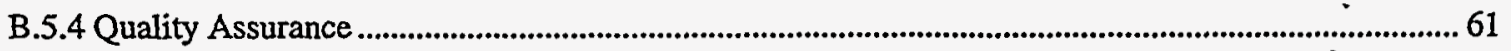

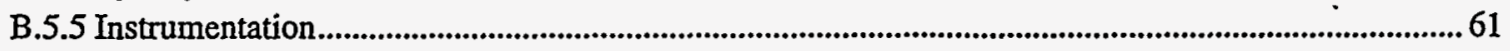

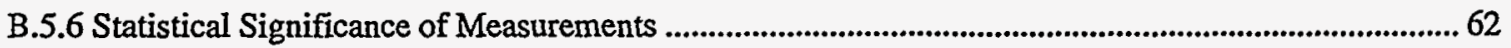

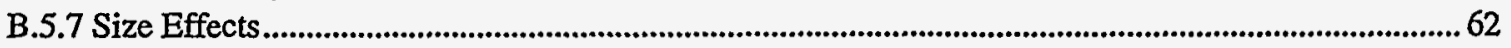

B.5.8 Test Confirmation of Scale Model and Scaling Laws ..................................................................62 62

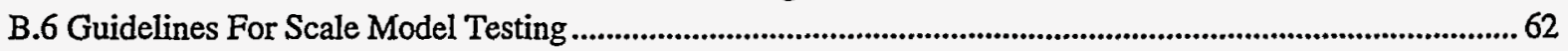

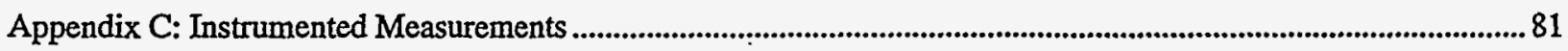

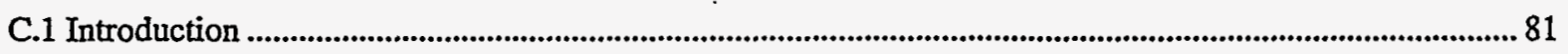

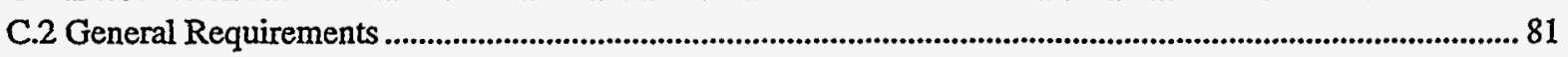

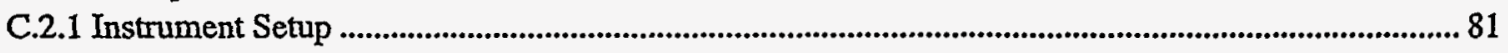

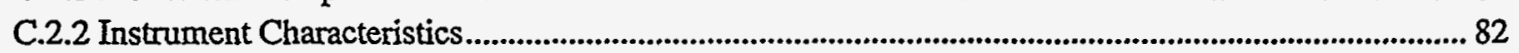

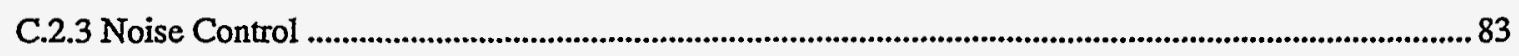

C.2.4 Recommended Practices ............................................................................................................... 84

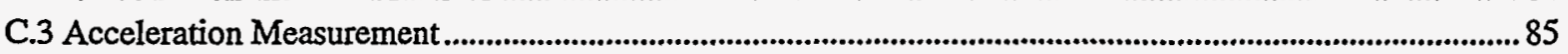

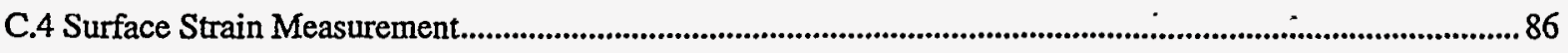

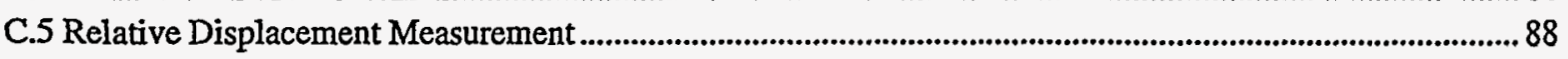

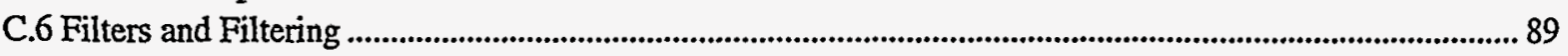

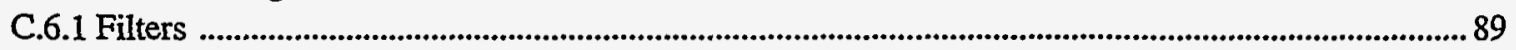

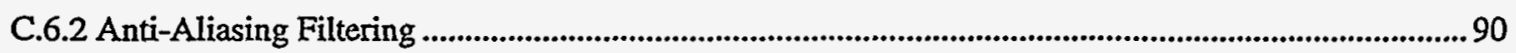

C.6.3 Separation of Package Responses by Filtering ............................................................................... 91 


\section{LIST OF FIGURES}

Figure 9-1 Axial acceleration time histories measured at locations near the top end of the DHLW cask during a 30-ft. bottom-end drop.

Figure 9-2 Axial surface strain histories measured at locations on the outer and inner surfaces of the DHLW cask during a 30-ft. bottom-end drop.

Figure 9-3 Axial acceleration time histories measured at locations near the top end of the DHLW cask during a 30-ft. top-end drop.

Figure 9-4 Time histories of axial bolt force, closure lid surface strain, and bolted closure joint opening of the DHLW cask measured during a 30-ft. top-end drop

Figure 9-5 Time histories of axial bolt force, closure lid surface strain, and bolted closure joint opening of the DHLW cask measured during a 40-in. puncture impact at the center of the closure lid ..................................33

Figure 9-6 Radial acceleration time histories of the DHLW cask measured during a 30-ft. side drop .........................34

Figure 9-7 Axial strain time histories of the DHLW cask measured during a 30-ft. side drop .....................................36

Figure A-1 Acceleration time history of impact responses.................................................................................................52

Figure B-1 Comparison of Impact.Response of Prototype and Scale Models of Rail Cask - 30-ft. End-On Drop,

Time History of Axial Impact Force ....................................................................................................................... 74

Figure B-2a Comparison of Impact Response of Prototype and Scale Models of Rail Cask with Lead Slump 30-ft. End-On Drop, Time History of Axial Impact Stress in Inner Container Shell ...................................... 75

Figure B-2b Comparison of Impact Response of Prototype and Scale Models of Rail Cask with Lead Slump 30-ft. End-On Drop, Time History of Axial Impact Stress in Outer Container Shell .....................................76

Figure B-3a Comparison of Impact Response of Prototype and Scale Models of Rail Cask with Lead Slump30-ft. End-On Drop, Time History of Hoop Impact Stress in Inner Container Shell ...................................... 77

Figure B-3b Comparison of Impact Response of Prototype and Scale Models of Rail Cask with Lead Slump30-ft. End-On Drop, Time History of Hoop Impact Stress in Outer Container Shell ................................... 78

Figure B-4a Comparison of Impact Response of Prototype and Scale Models of Rail Cask - 30-ft. Oblique Drop, Time History of Impact Forces and Momenta: Axial Force at Impact End of Container .................... 79

Figure B-4b Comparison of Impact Response of Prototype and Scale Models of Rail Cask - 30-ft. Oblique Drop, Time History of Impact Forces and Momenta: Bending Moment at Mid-Length of Container .......... 80

Figure C-1 Instrument Set-up for Transient Measurements..........................................................................................93

Figure C-2 Typical Design of Accelerometer ..............................................................................................................94

Figure C-3 Typical Frequency Response Curve of Accelerometer ..........................................................................95

Figure C-4 Typical Design of Wire-Resistance Strain Gage ...............................................................................96

Figure C-5 Wheatstone Bridge Circuits for Measurement of Resistance Change ........................................................99

Figure C-6 Variations of Wheatstone Circuit for Measurement of Strain ................................................................99

Figure C-7 Typical Design, and Output of Linear Variable Differential Transformer (LVDT) .................................99

Figure C-8 Frequency Response Characteristics of Butterworth Filter ............................................................................. 100

Figure C-9 (a) Sampling of Single-Frequency Analog Signal

(b) Relationship Among Signal, Sampling, and Alias Frequencies

(c) Fourier Spectrum of Multiple-Frequency Analog Signal

Figure C-10 Effect of Filter Cutoff Frequency on Acceleration Time History from Impact Test.............................. 102

\section{LIST OF TABLES}

Table 5-1 Scale Models for Drop Tests 13

Table 6-1 Common Non-destructive Examination (NDE) Methods ......................................................................... 18

Table B-1 Common Package Design and Expected Dominant Impact Response …..................................................6.64

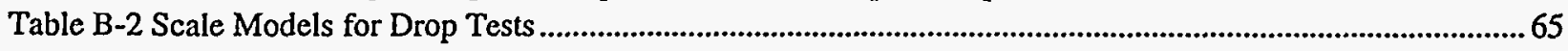

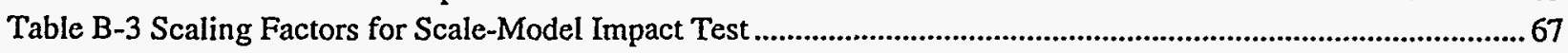

Table B-4 Scale Models Used in the SCANS Study ...........................................................................................72

Table B-5 Comparison of Rigid-Body Motions of Prototype and Scale-Model of Rail Cask .................................... 73 
This page left blank intentionally. 


\section{EXECUTIVE SUMMARY}

Federal regulation (10 CFR Part 71) requires that packaging for the transportation of radioactive materials be tested or evaluated under normal transport and hypothetical accident conditions which include a number of impact conditions (free drop, penetration, and puncture). This report provides guidance for the planning and implementation of the required impact tests. Specifically, the report discusses the major tasks that one should undertake, identifies the potential problems that one may encounter, and suggests possible approaches that one may take in carrying out the required impact tests. The report also provides the necessary technical background and justifications for its suggestions to help the reader understand and apply the recommendations. The report provides guidance for an impact test program with various objectives (compliance, benchmark, or development tests). The report covers the following topics: factors affecting the test plan; the selection of test conditions; the design of test models; pre- and post-test measurements, inspections, and acceptance tests; test measurements, instruments and analyses; test procedures, records, and reports. Sample impact tests and results from the open literature are discussed to illustrate the ideas presented in the report.

The report shows that the required impact tests on shipping packages are not a set of routine acceptance tests. Without the radioactive contents, regulatory compliance of the package can only be demonstrated indirectly using some test results from the impact tests and analyses relating the test results to the containment, shielding, and subcriticality performance of the package. There seem to exist as many approaches to the problem as packages. The approach used affects the test plan. The test plan should have details of required test measurements and observations. Measurements are essential to (1) demonstrate the package performance, (2) understand the impact behavior of the package, and (3) justify the approach. The required sophistication of the tests depends on the test objective and the package design.

Naturally, a compliance impact test program aimed at demonstrating regulatory compliance by test alone has the most requirements. It should have an adequate quality assurance program to ensure that:

- all test models be prototypical

- a sufficient number of tests to cover all critical impact conditions and other uncertainties

- sufficient measurements to understand the package impact behavior and to describe the impact damage

- proper criteria to demonstrate the compliance of the package with the regulation

Packages with a harder impact limiter tend to show more complex impact response involving significant wave and vibration responses. The impact tests on these packages require more extensive testing and have greater difficulty in predicting the outcome. Similarly, packages made of material which is not homogeneous and isotropic or those which have unstable failure modes such as buckling and brittle fracture require greater care in tests and data interpretation. In these cases, a few tests would not be sufficient to cover the large number of uncertainties.

Package responses that are affected by ill-defined, poorly understood, or difficult-to-reproduce parameters should be tested using full-scale rather than reduced-scale models. The above-mentioned buckling, and brittle fracture failures are examples. The leakage of contents through a bolted joint, the mechanical properties of honeycomb and concrete materials are additional examples.

Selecting the most critical test conditions demands a good understanding of the package behavior during impact. A condition that delivers a large amount of impact energy to a weak location in the package is a potentially critical condition to be tested. The actual test condition should be verified using photometric or other measurements obtained during a test. QA inspections or acceptance tests of the test model should be 
performed as required by the design specifications of the test package. For meaningful comparison, the same measurements and acceptance tests should be carried out before and after each test.

Visual inspection of damages and deformations is essential and convenient. However, quantitative measurements are more objective and amenable to analysis. For a package to be in compliance with the regulations or the test acceptance criteria, the package analysis must be based on quantitative measurements like leakage rates, component deformations, etc. Instrumented measurements obtained during the test, like accelerations, deformations, surface strains, temperatures, and pressures are not essential except in a benchmark test, where response time histories are used to verify computer programs. However, instrumented measurements can be used in all tests to gain additional understanding of the package behavior, to aid the interpretation of the test results, to justify the test conclusions, and to demonstrate the margin of safety of the package. For all free-drop tests, it is recommended that sufficient acceleration data be collected to provide a reasonably accurate measurement of the rigid-body impact acceleration of the package. In most packages, the rigid-body or the quasi-static response dominates the impact response. Thus, the acceleration data provide a good indication of the load and stress level received by each component of the package.

Test procedures should be written to ensure the precision, quality and safety in the execution of the test plan. Raw data should be preserved to allow re-evaluation in case differences appear in the interpretation of the data. Accurate records of the test results and subsequent analyses should be kept to support the conclusions of the test. The test report should justify as well as describe the test setup; the test conditions; the test model; the pre-test, post-test, and in-test measurements and inspections. Conflicting results should be identified and explained. The magnitude of possible errors should be estimated. Findings should be supported by direct test evidences or sound arguments. 


\section{PREFACE AND ACKNOWLEDGMENTS}

This report contains recommended practices for conducting impact tests on shipping packages used for the transport of radioactive materials. The work, funded by the Storage and Transport Systems Branch, within the Office of Nuclear Material Safety and Safeguards (NMSS) of the U.S. Nuclear Regulatory Commission (NRC), took place at Lawrence Livermore National Laboratory (LLNL). D. Tiktinsky was the NRC Project Manager and H. W. Lee was the NRC Technical Monitor for the project.

Recommendations set forth are the results of applying existing knowledge of impact testing to the special needs of packaging for the transportation of radioactive materials.

The authors are indebted to several individuals for their invaluable contributions to this work: $H$. W. Lee of the NRC for the overall technical guidance; R. Hafner, J. Hovingh, T. Lo, E. Russell, W. M. Shay Jr., and J. VanSant of LLNL for their consultations; R. Pomares of General Electric Company, M. Koploy and A. Zimmer of General Atomics, W. L. Uncapher of Sandia National Laboratory, and J. Hovingh of LLNL for providing details of the sample tests cited in this report. The authors also wish to thank the following LLNL staff: Ms. Lyssa Campbell for editing, and formatting and Ms. Rosa Yamamoto for administrative support. 
This page left blank intentionally. 


\section{INTRODUCTION}

\subsection{Objectives and Approach}

Federal regulation (10 CFR Part 71) requires that a shipping package for the transportation of radioactive materials is to be tested by free drops. The free drop tests, which this report will generically refer to as impact tests, include free drops of the package onto (1) an unyielding surface, (2) a puncture bar (puncture), and (3) free drops of a small cylinder onto the package (penetration). At present, standards do not exist to guide package designers in planning and conducting these impact tests. This report attempts to fill the need by establishing guidelines for conducting impact tests.

The report does not focus on a specific package design nor a specific impact test. Instead, it concentrates on the establishment of general principles and techniques which can be applied to all package designs and all impact tests. The reported information came from a critical review of existing literature on package drop tests and from an application of existing knowledge of impact (or shock) testing to the specific test and design needs of shipping packages. The report covers the requirements for all stages of an impact test program, including planning, conducting, recording, analyzing, and reporting the tests. For each step, the report highlights the necessary considerations, and potential problems encountered. The report attempts to identify the causes and suggest possible solutions for the encountered problems. This report, however, is not intended to be a technical reference nor a comprehensive guide for finding the solutions to problems encountered. The reader of this report should always depend on specialized technical references for this purpose.

The report contains twelve sections:

- Section 1 discusses the regulatory impact tests. The section identifies the difficulties in conducting these impact tests, which the remainder of the report addresses.
- Section 2 discusses the factors which determine an impact test plan.

- Section 3 suggests criteria to be used for the selection of critical test conditions.

- Section 4 discusses acceptance criteria and tests which can be used to demonstrate the compliance of a tested package with the regulation.

- Section 5 describes the essential features for test models including partial and scale models.

- Section 6 specifies inspections and acceptance tests to be performed before and after each impact test.

- Section 7 identifies test measurements and describes the methods and problems.of instrumented measurements.

- Section 8 emphasizes the importance of complete and accurate test records.

- Section 9 discusses data analysis.

- Section 10 discusses the need for written test procedures.

- Section 11 outlines the contents of a typical test report.

- Section 12 concludes the report by summarizing all essential aspects of an impact test program.

\subsection{Federal Regulation}

Federal regulation 10 CFR Part 71 requires that a package manufactured for the transport of radioactive materials demonstrate its ability to maintain containment, shielding and subcriticality of its contents. The demonstration is to be performed under a specified set of normal transport and hypothetical accident conditions, which include the free-drop, penetration, and puncture impact conditions considered in this report. The regulation explicitly specifies all parameters of the impact conditions except the drop orientation and the impact location. For these 
unspecified parameters, the regulation requires that they be selected to inflict the maximum damage to the package. The damage is in the package ability to maintain containment, shielding and subcriticality of its radioactive contents to meet the acceptable limits specified in the regulation under the normal transport and hypothetical accident conditions.

The planner of an impact test program faces two major challenges: (1) identify the most damaging impact conditions to be tested, (2) demonstrate how the containment, shielding and subcriticality of the radioactive contents of the package is in compliance with the regulation.

To minimize the number of tests, the planner should believe that the selected tests are among the most critical. To comply with the regulation, the planner should also be prepared to convince others that all critical conditions have been included. For this task, he can rely on analyses and engineering judgment. However, test data obtained directly from the impact tests can be proven more objective. He should consider taking special measurements during the tests to provide better understanding of the package behavior. Section 3 will discuss the various approaches to selecting test conditions.
The use of actual contents or radioactive materials in impact tests is too risky and is discouraged. Therefore, the package performance of containment, shielding, and subcriticality cannot be directly measured and compared to the corresponding limits set forth by the regulation. The planner of the impact test program has to propose alternative measurements and criteria to demonstrate the performance indirectly without using radioactive materials. To claim compliance with the regulation, the planner has to prove that the proposed performance criteria are quantitatively equivalent to or exceeding the regulatory standards. As in the "selection of test conditions, the planner can use supportive analyses or tests to prove compliance. Section 4 will examine the various approaches to this problem.

The impact test plan-including the test. conditions and the acceptance criteria-can vary significantly with the package design and behavior. The next section (Section 2) describes some of the factors and their effects on an impact test plan. 


\section{TEST PLANNING}

\subsection{Basic Considerations}

Planning is the single most important step of a test program, because it determines not only the cost but also the success of the test program. A test plan has even greater significance when conducting impact testing of shipping packages for the transport of radioactive materials because of the challenges associated with selecting the most damaging test conditions and developing the acceptance criteria. As discussed in Section 1, the test plan for a shipping package needs to address the basis for selecting both the test conditions and the acceptance criteria. The test plan also needs to include methods for obtaining test results to support the selection.

Many factors affect the details of an impact test plan. The primary factors are the test objective, the package design, and the impact behaviors of the package structure and materials. The following subsections (Sections 2.1.1 through 2.1.3) discuss the potential effect of each of these factors on the test plan. Subsection 2.2 summarizes the general requirements for an adequate test plan.

\subsubsection{Test Objective}

Section 1.2 points out that regulatory compliance can only be indirectly demonstrated without using radioactive contents. The results from an impact test program can be used in many different ways to demonstrate the compliance indirectly.

Therefore, different impact test programs can have different immediate objectives, even though they contribute to the same final goal of demonstrating regulatory compliance. In general, the test programs can be divided into three groups according to their specific objectives, namely, the exploratory (or development) test, the compliance (or certification) test, and the benchmark test. These test programs can have significantly different requirements and test plans because of their differences in objective.

Development test group

The development test group includes tests used to evaluate package design concepts or to obtain a qualitative or quantitative understanding of the package behavior under impact. Test results are often used to support the claim that the package design is in compliance with the regulation. The tests in this group are usually performed prior to the development of a test model for benchmark or compliance tests. The primary objectives of these tests are to identify the dominant physical phenomena and model features in order to justify the test models and plan for benchmark and compliance tests. These tests usually do not have to meet specific criteria. The test results can even be qualitative or relative (i.e., the results can be visual and used to compare with those obtained with different models), as long as they provide useful information.

\section{Compliance test group.}

The compliance test group includes all test programs intended to demonstrate, by test alone, the compliance of a package with the regulation. Obviously, the impact tests in these programs should be performed using prototypical models (or specimens). Any deviations in the design, fabrication, and quality assurance of the model from the actual package will require justification or appropriate compensation. As stated in Section 1.2 , one unavoidable deviation is the use of nonradioactive simulated contents in the test model. To accommodate this deviation, equivalent performance parameters and acceptance criteria, like the limit for the helium leak test on the containment vessel, have to be developed and used in lieu of those specified in the regulation. In theory, only the equivalent performance parameters need to be measured in the impact tests of this group. However, in practice, other measurements are often needed to defend the selection of the acceptance criteria and test conditions. Additional measurements are particularly desirable for large or complex packages, which are costly to produce and test. The additional measurements often help reduce the total number of tests and cost.

\section{Benchmark test group}

The benchmark test group includes all test programs used to justify the analysis method or 
model used to demonstrate the compliance of a shipping cask. Test models for these test programs need not be prototypical and can be greatly simplified; they only need to retain the design features expected to dominate the impact response of the package. For example, to obtain the forcedeflection curve of the impact limiter to be used in a package impact analysis, it is justifiable to use a simplified package model, containing only the impact limiter to be tested and a rigid mass to represent the remainder of the package. With proper justification, partial models, and reducedscale models can be used. Moreover, the choice of test conditions is also not critical, as long as they induce the same impact response and failure modes of the package so that the ability of the analysis method or model for predicting the response and failures can be validated by the test results. Instrumented measurements of the package response (acceleration, surface strain, etc.) at several locations during an impact test are essential to benchmark tests; the results must be compared to corresponding analysis results to justify the analysis method or model.

In view of the dependence of the test plan on the objective, it is essential to start the planning with a set of clearly defined objectives.

\subsubsection{Impact Response and Package Design}

An impact can excite three dynamic responses of a package structure: the wave, the vibration and the quasi-static-deformation responses. All three responses contribute to the package response. Depending on the package design and the drop orientation, any one or a combination of these responses can cause damage to the package structure. Most package designs have soft impact limiters. The impact response of these packages is dominated by the quasi-static deformation response. In packages with hard impact limiters, the wave and vibration responses are also significant. Recognizing the significant responses and their characteristics is a key to the successful planning of an impact test program.

Of the three impact responses, the wave appears first at the start of the impact. It is caused by the sudden change of velocity (or momentum) of the package material at the impact area. The wave is a messenger or distributor; it carries the sudden momentum change from the impact area to other. parts of the package and eventually distributes the impact energy to the entire package after multiple reflections and refractions. The vibration response is the result of the package structure responding to the impact in the natural vibration modes of the package. A package has an infinite number of vibration modes. However, only the modes with (1) the frequency closest to the excitation frequency, (2) the lowest frequency, and (3) a mode shape closest to the spatial distribution of the excitation are sensitive to the excitation and show appreciable response. The quasi-static deformation response is the result of the wholebody or rigid-body motion of the package caused by the impact. The rigid-body acceleration at the center of gravity of the package determines the response. The total impact force of the quasi-static response is determined by the rigid-body acceleration and the total mass of the package. The distribution of quasi-static deformation and stress in the package is determined by the distribution of acceleration and mass in the package.

Appendix A discusses the cause, characteristics, effects and interrelationship of the three dynamic responses. Based on the discussion, Appendix A identifies the following test requirements for the impact responses:

The test model should adequately simulate the design features affecting the dominant impact response of the package. The wave and vibration responses need a more precise distribution of mass and stiffness properties in the test model than the quasi-static response. They also require a better reproduction of the mechanical behavior and impedance at the component joints of the model. For example, a welded joint should not be replaced with a bolted joint. An effective way to determine the adequacy of a model for wave and vibration responses is to perform a modal survey of the test model. If the model can reproduce the dominant vibration frequencies of the package, the model is adequate.

The critical test condition varies with the impact responses. The selection of the test condition for the quasi-static response should be based on the 
impact force (or deceleration) and the static deformation of the package. The worst condition should produce the highest impact force in the weakest orientation of the package. The selection of the test conditions for the vibration should be based on the vibration frequencies and modes of the package. The worst condition should produce the highest vibration amplitude of the package. The test conditions for the wave response should be based on wave propagation characteristics. The worst condition should generate the highest amplitude wave propagating in a direction with minimum attenuation.

The post-test inspection should cover locations where damages caused by the dominant responses tend to occur. The area near the impact point and the weak structural components of the package should be inspected for damages caused by the quasi-static response. If the wave and vibration responses are significant, the inspection should be expanded to include component interfaces and structural joints.

The frequency response of the measurement instruments should be adequate for the impact response to be measured. Of the three responses, the wave response has the highest frequencies. The frequencies of the vibration response are lower, and the frequencies of the quasi-static response are usually the lowest. An instrument for measuring all three impact responses would require a wide frequency range extending from 0 $\mathrm{Hz}$ up to a high frequency limit determined by the wave and vibration responses.

\subsubsection{Structural Failure Modes and Material Properties}

Other factors which can significantly influence the test plan are failure modes and material properties. Some of these factors would require more details to be included in the test model and may severely limit the use of scale and partial models.

Buckling failures are common in shipping casks using fin and honeycomb impact limiters. The failures are known to be sensitive to geometric imperfections, residual stresses and boundary conditions. These failure characteristics have severely limited the use of scale models and partial models for impact tests where buckling is expected to occur.

Similar to buckling failures, brittle fractures also depend on many ill-defined and difficult-tocontrol parameters. Brittle fracture failures depend not only on local geometries and stresses but also on the size and nature of existing flaws in the hardware. Thus the results of a scale-model test should not be used to demonstrate brittle fracture resistance of a package.

The leakage through a bolted closure joint also depends on many ill-defined and difficult-tocontrol parameters. The use of a scale model is , not generally accepted for leak tests. Scale models are also impossible for materials with strain-rate dependent properties and for thermal tests, because proper scaling laws can not be developed. Scale model tests of materials with large microstructures like reinforced concrete, honeycombs, etc. have also met with little success.

A full-scale prototypical test model should be employed for the study of the special situations discussed here. Section 5 will discuss further the use and limitations of scale models.

\subsection{General Requirements}

The plan for an impact test program should be documented. A complete plan should contain two parts: the first part contains general information which should include the objectives, the basis, and the acceptance criteria for the test results; the second part should provide details which specify precisely the test conditions, procedures, measurements, and standards to be used. The first part is needed to justify the adequacy and applicability of the test plan and the second part is needed to ensure the quality of the test results. 


\section{Test Planning}

The background information given in the foregoing sections (2.1.1 through 2.1.3) can be used to develop the first part of the test plan. Discussions on the contents of the second part follow and will be divided into the following areas:

- The test conditions;

- The test acceptance criteria;

- The design and fabrication of the test model;

- The methods and standards of pre- and posttest inspection/examination of the test model;
- The type and number of test measurements;

- The acquisition and analysis methods for the test measurements;

- the required test records and reports.

The following sections ( 3 through 9) discuss the basic considerations, requirements and difficulties in each of these areas. 


\section{TEST CONDITIONS}

\subsection{Basic Considerations}

A complete set of test conditions should include both the initial conditions and the impact conditions. The initial conditions specify the physical and mechanical conditions of the test model at the beginning of the impact test which should include temperatures, moisture contents (if important to the material property), deformations (if a damaged specimen is used), and pressures. The impact conditions specify the kinematic conditions at which the impact is to occur. It should include the drop height, the drop orientation, and the impact location of the test model. Both the initial and impact conditions should be selected during the test planning.

The objective of the test program, the package design, and the potential package failure modes dictate the selection of the impact test conditions. As discussed in Subsection 2.1.1, if the test program includes a compliance test, the most critical or damaging test conditions should be identified and tested. As for test programs which include benchmark and exploratory tests, testing under the most critical conditions is not essential, since they are not intended to directly demonstrate compliance with the regulation.

To find critical test conditions, potential failure modes (or probable failure scenarios) should first be identified. Then the most unfavorable initial and impact conditions to the identified failure modes can be selected as the test conditions. The identified failure modes should be considered for safety-related components of the package, which include all those components responsible for reducing the effects of impact, thermal or pressure load on the package, and for maintaining the containment, shielding, or subcriticality capability of the package.

The package material properties usually dictate the choice of initial conditions, as material properties are sensitive to temperature and other environmental conditions. Both (high and low) extremes of the environmental conditions should be considered, since the outcome is sometimes difficult to predict. High temperatures have the unfavorable effect of weakening the material but the favorable effect of reducing the impact load. Low temperatures have similar mixed results; they harden the material but increase the impact load and, in some materials, may induce brittle fractures. For materials which exhibits a dramatic reduction in ductility at low temperatures like the carbon steels, the low temperature limit should always be considered as a critical test condition. In materials whose properties depend on moisture contents, like wood for impact limiters, the extremes in moisture contents should be considered.

The impact condition should be selected based on its potential to produce damage. The damaging potential of an impact depends not only. on the intensity of the impact but also on the effectiveness of transferring the impact energy to a potential failure mode of the package. An impact that can deliver the maximum amount of . energy to a vulnerable area of the package should be considered a candidate test condition. Each failure mode may have its own test conditions. For example, the test condition for the spallation damage of closure bolts discussed in Appendix A is the end-on impact at the bottom of the cylindrical package. The test condition for a local buckling failure of the cylindrical wall may be an oblique or a side impact where the bending moment generated by the impact force may cause a high compression on one side of the cylinder. The test plan should identify the potential failure mode used to select each test condition so that the test team knows the purpose of the test and focuses their inspection and analysis efforts on the areas of concern.

Applying the principles identified in this subsection, the next subsection suggests some critical impact conditions to be considered.

\subsection{Critical Test Conditions}

Following are impact conditions which are likely to produce an impact of significant intensity or consequence: 
- Direct impact on a safety-related component of the package (e.g., thermal insulation, closure bolts, valves, and penetrations of containment boundary)

- Direct impact on a geometric and material discontinuity or other structural weakness (e.g. welds, component joints, or locations of thickness change.)

- Impact on a large surface area of the package (e.g., an end-on drop)

- Impact on a hard surface area of the package (e.g. a drop on a lifting lug)

- Impact on package areas un- or underprotected by impact limiters

- Impact with full package weight over a small impact area (e.g., a c.g.-over-corner drop)

- Impact in a weak direction or position of the safety-related components (e.g., a side drop of a long cylindrical container protected by impact limiters located at its two ends)

- Impact producing highly localized damage (e.g., puncture drop near a material or geometric discontinuity which prevents the impact energy to be absorbed by the permanent deformation of a large area)

\subsection{Number of Tests}

In theory, only one test is needed for each critical test condition. Thus the total number of tests in an impact test program equals the total number of critical impact conditions, which in turn equals the total number of possible failure modes of the package. However, in practice, the program may need considerably more tests than the critical impact conditions because large uncertainties exist in the selection and implementation of the test conditions. The uncertainties can be caused by one of the following situations:

- A complex package design whose impact behavior is difficult to predict;

- A sensitive failure mode (or mechanism) whose causes are many and difficult to control;

- A package that lacks strict quality control in the design and fabrication;

- A test setup that lacks precise control of the test conditions and measurements.

A good test plan should include duplicate tests to demonstrate the possible scatter of the test results due to these causes. The plan should be flexible so that if the test data indicate, additional tests can be included. Increased understanding of the package behavior can help reduce the number of tests. The needed understanding can be acquired by performing impact stress analysis of the package or by monitoring the package response during the impact test using proper instruments. 


\section{TEST ACCEPTANCE CRITERIA}

\subsection{Basic Considerations}

Test acceptance criteria consist of two parts. The first part can be called the test criteria and the second part standards. The test criteria are performance limits (deformation, damage, leakage, etc.) which are parameters used to evaluate the test results and determine whether or not the tested package passes the test (or complies with the regulation). The standards refer to all engineering codes, practices and standards specified for controlling the various operations in the test programs. Standards should be identified for fabricating and inspecting the test packages, the measurement of the test results, the calibration of the measurement instruments, etc. The standards do not directly determine the acceptability of the test package but they affect the quality, reliability, and, consequently, the acceptability of the test findings.

All test programs should have standards to assure the quality of the test results. A compliance test program (as defined in Subsection 2.1.1) should also have a set of test criteria to demonstrate the compliance of the package with the regulation.

Both the standards and the test criteria should be defined in specific, measurable quantities and limits, so that no ambiguity exists in their interpretation. However, general statements can be acceptable. The test criteria of "no permanent deformation" implies that the measurable dimensions of the tested package must not change. In practice, the criteria should be supplemented with a list of specific dimensions to be measured. Subsections 4.2 and 4.3 discuss further the requirements and basis for the standards and the test criteria.

\subsection{Procedures and Standards}

Standards and limits should always be specified for the following test operations:

- Model fabrication

- Model inspection/examination
- Instrument calibration

If a prototypical test model is used, the same tolerances, codes and standards used for fabrication and inspection of the production package should also be used for the test model. If a scale model is used, an equivalent set of standards and limits should be developed in accordance with the scaling law. The procedures for calibrating instruments and equipment should be developed, if they are not specified by the manufacturer. The conditions used for the calibration should be comparable to the test conditions.

\subsection{Test Criteria}

The test criteria to demonstrate compliance with the regulatory requirements are package dependent and thus must be developed and justified for each package.

Subsection 1.2 pointed out that without using the actual radioactive contents in an impact test, the compliance of a package with the regulatory requirements for containment, subcriticality and shielding of the radioactive contents can only be indirectly demonstrated using equivalent performance parameters and acceptance criteria. The equivalent parameters are measurable mechanical or physical parameters not involving the radioactive contents. They can be the deformations of the safety-related components of the test model. They can also be parameters measured using a performance acceptance test like the helium leak rate of the containment vessel. The acceptance criteria are specified in term of these performance parameters. Of course, the test acceptance criteria need to be proven to meet or exceed the acceptable limits specified in the regulation. Appropriate containment, criticality and shielding analyses of the package can provide the necessary proof.

Similar to the regulatory acceptable limits, the test criteria can specify general or specific limits on the package performance. The following sample 
criteria have been used in past compliance test programs:

- No obvious structural instability or rupture observed in visual inspection or in radiographic examination;

- No deformation exceeding the limits set by the containment, subcriticality, and shielding analyses;
- No indication of imminent or hidden failures as shown by measured stress/strain levels, or by post-test examinations;

- No noticeable change of performance as demonstrated by leak tests conducted before and after an impact test.

Sections 6 and 7 describe the typical methods for the necessary inspections, measurements, and acceptance tests. 


\section{TEST MODELS}

\subsection{Basic Considerations}

The test objective, potential failure mode, and impact behavior of the package determine the extent and details of the test model. The model for a compliance test (as defined in Subsection 2.1.1) should be prototypical. The model for a test used to benchmark an analysis should be identical to the analysis model. Regardless of the objective, the model should include all the parts and details of the package that have appreciable effects on the failure mode and impact behavior to be demonstrated by the test.

Test models are expensive. The use of partial, simplified, and reduced-scale models is encouraged, provided it can be justified. Even the prototypical model for compliance tests need not include all package details that are insignificant to the particular package performance to be demonstrated. However, the omissions or simplifications need to be identified and properly justified or compensated with respect to the package performance. The following subsections highlight the specific requirements for each model option. The discussion applies to the modeling of the package as well as the target used for the impact test.

\subsection{Prototypical model}

To minimize uncertainties and difficulties in relating the test results to the performance of the actual package, test models for impact test, especially those for a compliance test program, should be prototypical, which means that the test models and the actual package are identical in design and fabrication; the design includes the geometry and material; the fabrication includes the production processes, QA requirements and assembly procedures. The advantage of using prototypical models is obvious. Since the models are identical to the actual package within the allowable tolerances, the test results represent the performance of the actual package under the same impact conditions. No justification or extrapolation of the test results are needed.

However, the usefulness of the results can still be questioned as to how much the results can vary under the influence of the design tolerances and of the uncertainties in the selection and implementation of the impact test conditions.

\subsection{Partial Model}

Partial models can be used to demonstrate a specific capability of a shipping package. For example, the impact limiter can be tested alone to demonstrate its ability to reduce shock. Similarly, a bolted closure can be tested by itself to demonstrate its ability to maintain containment. However, it is essential to create in these tests the same impact conditions or loads as in the tests of the complete model. To this end, masses may be added to the partial models to preserve the total mass and the location of the center of gravity of the package. Compensations of this natire are common in the design of partial models. The development and justification of compensations require a thorough understanding of the impact behavior of the package. Sometimes, an understanding of the basis of the acceptance tests used to demonstrate the capability of the partial model may also be necessary. The omitted part of the package may have an effect on the test result.

\subsection{Simplified Model}

Even for compliance tests, simplifications of the test model are permissible and often necessary. Some details of the package may be simplified or omitted in the test model, if the details can be shown to contribute insignificantly to the package performance.

Benchmark tests used for verifying computer methods or models often employ simplified models. For this application, strong justifications are not needed if the same simplified package is tested and analyzed. The only requirement of the simplified model is that it simulates all potential failure modes of the actual package under the impact conditions. For example, if buckling of the containment vessel is a possibility in the actual package, the simplified model for a benchmark test should include all package details which may 

Table 5-1 Scale Models for Drop Tests

\begin{tabular}{|c|c|c|c|}
\hline Model Destination & A-4 & B-2 & C-1 \\
\hline Model Capability: & & & \\
\hline $\begin{array}{l}\text { Impact response not } \\
\text { simulated }\end{array}$ & None & Wave and Vibration & Wave and Vibration \\
\hline $\begin{array}{l}\text { Material effect not } \\
\text { included }\end{array}$ & Gravity and Strain-rate & Stiffness & Mass \\
\hline Model design: & & & \\
\hline $\begin{array}{l}\text { Independent scaling } \\
\text { factor }\end{array}$ & Length, $\mathrm{s}_{\mathrm{L}}$ & $\begin{array}{l}\text { Length, } \mathrm{s}_{\mathrm{L}} \\
\text { Time, } \mathrm{s}_{\mathrm{t}} \\
\text { Mass, } \mathrm{s}_{\mathrm{M}}\end{array}$ & $\begin{array}{l}\text { Length, } \mathrm{s}_{\mathrm{L}} \\
\text { Mass, } \mathrm{s}_{\mathrm{M}}\end{array}$ \\
\hline $\begin{array}{l}\text { Dependent scaling } \\
\text { factor }\end{array}$ & $\begin{array}{l}\text { Time }=s_{L} \\
\text { Mass }=s_{L}^{3}\end{array}$ & & Time $=1$ \\
\hline $\begin{array}{l}\text { Relation of model and } \\
\text { prototype materials }\end{array}$ & Identical & Same mass property & Same stiffness property \\
\hline
\end{tabular}

$\mathrm{S}_{\mathrm{L}}=$ geometry scaling factor

adequate for use in packages with strain-rate sensitive materials like some foams, or for drop tests where the impact inertial load is not significantly higher than the weight of the package (i.e., the rigid-body acceleration of the package is not significantly higher than the gravitational acceleration).

Being the only general-purpose scale model, Model A-4 has been used for most scale-model drop tests of shipping packages in the past. The key features of a drop test using this model are as follows:

- The model is totally similar to the prototype in geometry (i.e., an undistorted scale model) and is made of identical material as the prototype.

- The model has only one independent scaling factor; the scaling factor for length or the geometry scaling factor $\left(s_{L}\right)$ determines the scaling factor for all other parameters.

- The drop conditions (i.e., the drop height, velocity and angle) used to test the scale-model are identical to those of the prototype.
- The stresses and strains in the scale model and in the prototype are identical.

- The displacement/deformation and impact duration of the scale model are proportional to the geometry scale of the model.

- The (translational) rigid-body acceleration and the total impact force in the impact area of the scale model are inversely proportional to the geometry scale and to the square of the geometry scale of the model, respectively.

Accordingly, a half-scale model A-4 of a package dropped from a height of $30 \mathrm{ft}$. would have one half the deformation and impact duration, the same stress and strain, two times the rigid-body (translational) acceleration, and one fourth the impact force of the package dropped from the same height.

It is permissible to use different scale models for different parts of the package; each model simulates only the dominant property and impact response of the modeled part. For example, in a package with soft impact limiters, the impact behavior of the package is usually dominated by 
the quasi-static impact response, which is determined by the relatively large mass of the container and contents and by the relatively low stiffness of the soft impact limiters. Accordingly, Model B-2 can be used for the container and contents, and Model C-1 can be used for the impact limiter in the drop test model used for obtaining the rigid-body acceleration of the package. This composite scale model has been used in the past for this purpose and for obtaining the force-deflection curve of the impact limiter.

Even if a scale model can be designed in theory, the scale model may still encounter significant difficulties in practice. Some common difficulties are as follows:

- The fabrication method may not be scaled. The scale model may have different material properties, because of the use of different fabrication method from the actual package.

- The same fabrication method may affect the material properties differently in models of different scale (or size).

- The scale model may not completely reproduce the package details which significantly affect the impact behavior and failure modes of the package.

- The transducers or instruments for measurements may not fit into a reduced-scale model or may appreciably affect the response of the reduced-scale model.

To minimize the practical difficulties, scale models used for impact tests of shipping packages in the past were mostly between quarter- and halfscale.

Based on the discussions of this subsection and of Appendix B, a scale model impact test is not recommended for the following situations:

- Drops producing low impact (g) loads compared to the gravity load (e.g., 1-ft. drops on soft impact limiters)

- Package made of strain-rate sensitive materials (Some materials used for the construction of shipping packages, such as lead and foam, show very different stress-strain relationships when they are test under static and dynamic conditions (Chun et al., 1.991; Maji et al., 1990). Nevertheless, the change of strain-rates generated by the use of a scale model may not be sufficiently large to cause a significant change in the stress-strain relationships. Therefore, these materials can be practically treated as strain-rate independent materials for scale model tests, especially when the model used is near full scale.)

- Package made of materials having a coarse microstructure (e.g., reinforced concrete, honeycombs, or wood composites)

- Drop tests to demonstrate the leak tightness of bolted closure joints

- Drop tests to demonstrate structural and material failure modes which are known to be sensitive to microscopic or local conditions of the package (e.g., the fracture toughness, and certain buckling strengths of a package)

For these situations, the adequacy of scale models should definitely be confirmed experimentally or analytically as follows:

- Obtain test results using scale models of at least two different sizes and show that corresponding results of the scale models can be correlated using the established scaling laws.

- Analytically predict the test results of a scale model to show that all parameters controlling the test results have been identified and properly included.

The analytical approach can also be used to validate the analytical method or model used for predictions under other test conditions.

\subsection{Unyielding Target and Puncture Bar}

The regulation requires that the target for a free drop test of a shipping package be an unyielding surface. To meet this requirement, the target for a free-drop test should have minimal deformation and motion compared to the deformation of the package, so that almost all of the impact energy is consumed in the deformation of the package and 
very little in the deformation and motion of the target. To this end, the target should have the following properties:

- Large mass and adequate support to minimize the rigid-body motion of the target

- Sufficient thickness relative to the width and length to minimize the global bending deformation of the target

- Sufficient surface hardness to minimize the local target deformation at and around the impact area

- Proper shape and construction to ensure internal strength and integrity of the target and to minimize waves and vibrations in the target

The International Atomic Energy Agency (IAEA) Safety Series No. 37 has given the following example of an unyielding target for the package drop test:

"A steel plate as the upper surface of a concrete block. The combined mass of the steel and concrete shall be at least 10 times that of the specimen to be dropped on it. The block shall be set on firm soil and the steel plate should be at least 4-cm thick and floated onto the concrete while it is still wet. The plate should have protruding fixed steel structures on its lower surface to ensure tight contact with the target. Since flexure of the target is to be avoided, especially in the vertical direction, it is recommended that the target should be close to cubic in form, with the depth comparable to the width and length."

Most targets for the drop test are similar to the IAEA example.

The target for puncture tests (a 6-in diameter steel bar) must be scaled as if it were a part of the test model. The scaling of the puncture bar is needed to maintain the similarity of the impact behaviors of the prototype and the scale model especially in and around the impact area. The impact response of the impact area depends on the geometries and the mechanical impedances of the impacting bodies near the impact point. The same scaling laws should be applied to the models of the package and the puncture bar. A scale model puncture test, using the scale model A-4 defined in Table 5.1, will require that a scaled package model be dropped 40 inches onto the top end of a scaled puncture-bar model of the same scale factor, SL. 
5. Test Models 


\section{PRE- AND POST-TEST INSPECTIONS}

\subsection{Basic Considerations}

Every test model should be inspected before and after each impact test. The pre-test inspections serve two purposes: to ensure the quality of the test model, and to establish the pre-test condition of the model. The post-test inspection assesses the damage of the model by comparing the pre- and post-test conditions. The assurance of the testmodel quality is essential for all tests, although its extent can vary with the objective of the impact test program. For compliance tests (as defined in Subsection 2.1.1), the test model should meet all quality assurance requirements of the prototype to ensure that the test model and the prototype have equal quality (or the test model represents a typical production package). For benchmark and other tests, a test model only needs to show an adequate quality or a quality similar to other test models used in the same test program.

A complete inspection program may consist of visual inspections, dimension surveys, nondestructive examinations (NDE), and acceptance tests. Destructive examinations may also be applied after an impact test to gain additional information on the quality and damage of the model. Some of the NDE and acceptance tests have established industrial standards. These standards should be identified and used to control these operations. As suggested in Subsection 4.2, these standards should be part of the acceptance criteria of the test program. As pointed out in Subsection 2.1.2, the understanding of the design and impact response of the package should be applied to identify and minimize the number of critical locations to be inspected. The following subsections discuss the scope and necessary considerations of the inspection operations.

\subsection{Visual Inspection}

The visual inspection can be an effective and important method of examination, if it is carefully carried out by a professional. Using only simple tcols, an experienced inspector can help minimize the need for sophisticated examination by uncovering many fabrication defects and hidden impact damages.

In pre-test inspection, the inspector should reject test models with significant fabrication defects or damages. In post-test inspection, the inspector should record all significant impact damages like dents and scratches and identify the possible cause of the damages. If necessary, the inspector should also suggest locations for further inspection using NDE or other sophisticated methods. The effort should cover all safetyrelated components and focus on areas where fabrication defects and impact damages tend to appear. These areas include geometric and material discontinuities such as component boundaries, welds, joints, grooves, fillets and (external and internal) impact areas.

\subsection{Dimension Survey}

Dimension surveys should be conducted before and after an impact test to detect possible permanent deformations of the package structure caused by the test. The measurements can be compared to the deformation limits in the acceptance criteria to demonstrate the ability of the package to meet the requirements on containment, subcriticality, and shielding of the package contents. The surveys may also provide a quantitative understanding of the package response to the impact. An approximate estimate of the impact force, and the absorbed impact energy can be calculated from the measured permanent deformations.

Locations for dimension measurements should be determined before the test. The locations should be clearly marked, so that the same dimension can be measured and compared before and after the test to detect permanent deformation. The selected dimensions may include the global dimensions of safety-related components as well as the local dimensions near the external and internal impact areas where large distortions are expected. The global distortion of the components can provide some clues as to how the impact energy was distributed or absorbed in the package during the impact test. 


\subsection{Destructive Examination}

Destructive examinations can be made after an impact test to assess the extent and nature of the damage in the test package. Cross-sectional cuts of the damaged model can be made to show internal damages and the profile of the deformed areas. The conditions and probable causes of the damages can usually be determined by visual inspection. Ample photographs comparing the before and after conditions of the package should be used to report the results.

\subsection{Non-Destructive Examination}

Common non-destructive examination (NDE) methods include the dye-penetrant, magneticparticle, eddy-current, radiographic, and ultrasonic methods. They are methods to detect internal and surface flaws or cracks without damaging the inspected specimen. Table 6.1 briefly lists the capabilities and limitations of the methods. Non-destructive examination is mainly used to assure the fabrication quality of a finished product. For compliance tests (as defined in Subsection 2.1.2), the NDE methods specified for the actual package should be used on the prototypical test model to ensure that the test model has the same quality as the actual product. Therefore, the industrial codes and standards used for the design and fabrication of the package determine the NDE requirements. Code requirements are set to mitigate recognized risks of a design. For example, knowing that highstrength bolts are made of steels with limited ductility, and a bolt of large diameter is susceptible to brittle fracture, ASME Boiler and Pressure Code Section III specifies that bolts with a diameter exceeding 1 " are to be examined by one or more of the dye-penetrant, ultrasonic, and magnetic particle methods.

The same NDE method should be used before and after an impact test, if the post-test examination is necessary. The post-test examination should be imposed, if it is determined through visual inspection and knowledge of the material properties that the impact could have produced hidden flaws or cracks in the package materials. A material of limited ductility or fracture toughness can have additional flaws and cracks after the package impact test. A simple rule is to avoid the use of unsuitable materials and use materials permitted for class 1 components by the ASME Boiler and Pressure Vessel Code.

Table 6-1 Common Non-destructive Examination (NDE) Methods

\begin{tabular}{|l|l|}
\hline NDE Method & Application and Limitation \\
\hline Dye penetrant & For detecting surface cracks only \\
\hline Magnetic particle & $\begin{array}{l}\text { For detecting surface and near-surface cracks } \\
\text { Used for magnetic materials only }\end{array}$ \\
\hline Radiographic & $\begin{array}{l}\text { For detecting embedded impurities, defects, and } \\
\text { cracks }\end{array}$ \\
\hline Ultrasonic & $\begin{array}{l}\text { For internal cracks and thickness } \\
\text { May encounter special difficulties in austenitic } \\
\text { (300 series) stainless steels due to scattering } \\
\text { induced by the coarse grain structure of the } \\
\text { materials }\end{array}$ \\
\hline
\end{tabular}




\subsection{Acceptance Test}

Acceptance or proof tests are methods used to evaluate the overall performance or integrity of a finished product. For shipping packages, two common acceptance tests are the pressure test and the helium leak test of the containment vessel.

The regulation explicitly specifies the requirement of the pressure test; 10 CFR Part 71 Paragraph 71.85 states,' "Prior to the first use of any package ..., (b) Where the maximum normal operating pressure will exceed 34.3 kilopascal ( 5 psi) gauge, the licensee shall test the containment system at an internal pressure at least $50 \%$ higher than the maximum normal operating pressure to verify the capability of that system to maintain its structural integrity at that pressure." This requirement is similar to the ASME code requirement for the pressure test of boilers and pressure vessels.

The helium leak test for the containment system of a shipping package can indirectly demonstrate the compliance of the package with the regulatory requirement on the containment of the radioactive contents. The test produces a volume leak rate of the helium gas across the containment system boundary of the package, which is measured under a specified set of temperature and internal pressure. The American National Standard Institute (ANSI) standard suggests that if the helium gas leak rate of a container measured at a temperature of $25^{\circ} \mathrm{C}$ and pressure of $1 \mathrm{~atm}$. gauge is less than $2.96 \times 10^{-7} \mathrm{cc} / \mathrm{sec}$, the container can be considered practically leaktight. Unless the containment system of a package can qualify as a leaktight system, the leak rate measured from the helium leak acceptance test has to be first converted by justifiable means (analysis or others) into an equivalent leak rate of the radioactive contents of the package before compliance with the regulation can be demonstrated.

The helium leak test can be used to demonstrated the change made by an impact test on the performance or integrity of the package. If the same acceptance test is applied to the package before and after an impact test and shows a negligible change in the results, it is believable that the impact has negligible effects on the package performance and integrity. 
6. Pre-and Post-Test Inspections 


\section{TEST MEASUREMENTS}

\subsection{Basic Considerations}

Measurements made during an impact test can be grouped according to their objectives as follows:

- Measurements to verify the initial conditions of the package (temperature, internal pressure, etc.)

- Measurements to verify the impact conditions (impact velocity, impact angle, and position)

- Measurements to indicate the severity or intensity of the impact (the rigid-body (c.g.) acceleration, or impact force of the package)

- Measurements to meet specific needs of the test program (accelerations, stresses, strains, or deformations at specified locations of the package)

- Measurements to gain additional understanding of the package behavior during the impact (accelerations, stresses, strains, or deformations at locations selected to provide the necessary information)

The first three groups of measurements are essential for every test. Various factors can cause the actual conditions to deviate from the intended conditions. It is imperative to measure the actual initial and impact conditions of the test. The measurement of the impact intensity (i.e., the rigid-body acceleration or the impact force) provides a general quantitative description of the magnitude of the impact effect on the package. The information is needed to compare the damaging potential of different impact conditions, to confirm the choice of the test conditions, and to make crude estimates of the impact effect on various components and locations of the package. Subsection C.6.3 of Appendix C shows how the rigid body impact acceleration can be obtained by filtering the acceleration time history record of an impacting package, and how the acceleration can be used to estimate the impact force and stress in . the package.

The number of measurements in the fourth group varies with the objective of the test program.

Benchmark tests require measurements to compare with analysis results. Compliance and exploratory tests do not usually require measurements in addition to those in the first three groups. A general rule for the fourth group of measurements is that they should be directly measured as required. For example, if the stress at a specific location is required, the stress should be measured directly by mounting a strain gage at the location. The transient phenomena of impact render the interpolation of the result from stress measurements at adjacent locations almost impossible.

The fifth and last group of the measurements is intended to obtain a detailed, precise, quantitative description or understanding of the impact response of the package. Subsection 2.1.2 has described the package response to an impact as a combination of three responses (the wave, vibration, and quasi-static-deformation). Each response may dominant the package response at different times and locations. To obtain just a glimpse of the package response would require making the same transient (or time-history) measurements at several locations. Unfortunately, transient measurements are in general difficult and expensive to acquire and analyze. Therefore, the planner of an impact test program of a shipping package tends to avoid the measurements.

However, in large packages and packages with a complicated design, the total cost of an impact test can be lower with transient measurements than without. This is because the better understanding gained from the additional measurements can help justify the conclusion of the test program with fewer tests.

In principle, compliance tests (as defined in Subsection 2.1.1) do not need (time-history) test measurements during an impact test other than those required by the equivalent acceptance criteria (Subsection 4.3). In practice, however, the test measurements are often needed to explain or justify the selection of the test conditions, the reliability of the test results, and the reasonableness of the test conclusions. Occasionally, they are also needed to demonstrate the margin of safety of the package design or to 


\section{Test Measurements}

demonstrate that the package design has provided adequate assurance that it will perform satisfactorily in service. Since impact tests are expensive to conduct or repeat, it is advisable to consider all possible needs for test measurements in the planning of the test program.

High-speed photography is commonly used to record impact conditions. Special transducers and instruments are used to measure accelerations, strains and displacements. The requirements and considerations for these measurements are discussed in the following subsections.

\subsection{Photometric Measurements}

Video, high-speed framing, or streak cameras are used to photograph the impacting package model. From the photographs, the following photometric measurements can be made and used to verify the test conditions; justify the package design; or compare with other measurements:

- The impact velocity, angle, location, area, and duration of the package

- The rebound velocity, angle, and trajectory of the package

- Similar information for subsequent impacts and rebounds

- The rigid-body impact acceleration or g load of the package

- The deformation, and the absorbed energy of the impact limiter

- The force-deflection curve of the impact limiter

The resolution of photometric measurement increases with the speed of the camera. Cameras with a speed as high as $5000 \mathrm{fps}$ (frames per second) have been used to monitor drop tests. The high-speed photographs provide a complete record of the motion of the impacting package. The impact angle, impact position, impact duration and rebound height of the package, and the deformation of the impact limiter are directly measured from the photographic record. The impact velocity, acceleration, and force of the package, the force-deflection curve, and the energy absorbed by the impact limiter, are derived from the measured results. The rigid-body impact acceleration and force obtained from the photometric measurement are not accurate and are used mainly to support the measurement made using accelerometers.

For adequate photometric measurement, the following arrangements are essential:

- Photographs to be taken in two orthogonal horizontal directions with one of the directions normal to the package axis

- A time value displayed on each photograph

- A length scale on each photograph

- Clearly marked locations and grid lines on the package exterior surface including the c.g. location for motion measurements

- Adequate lighting or film/tape speed for the desired frequency of photography

- Minimization or proper control of parallax errors due to viewing the package at oblique angles

\subsection{Instrumented Measurements}

Continuous monitoring of accelerations, strains, relative displacements, or pressures at selected locations of a package during a drop test can provide significant insight into the package behavior. Unfortunately, these transient response measurements are not easy to obtain; they require special instruments and equipment. A detailed, individual account of the methods and instruments is beyond the scope of this report. Instead, the report focuses on providing general guidance. To this end, Appendix C identifies some common requirements, problems, and possible solutions for all instrumented measurements. The appendix contains a general discussion on topics applicable to all instrumented measurements: instrument setup, instrument characteristics, noise control, and recommended practices. Following the general discussion, Appendix $C$ is devoted to some key instruments used in impact testing of transportation packages: accelerometers, strain gages, linear variable differential transformers (LVDT), and filters. Although the appendix is not intended to be a comprehensive guide to 
instrumented measurements, it still provides considerable details especially in areas important to impact testing of packages. The following points highlight some of the significant guidelines for instrumented measurements:

- The selected instruments should have adequate stability, consistency, resolution, sensitivity, linearity, and frequency-response characteristics. The frequency range should adequately cover the frequencies of the dominant impact responses, which can extend from near $0 \mathrm{~Hz}$ for the quasi-static (rigid-body) response to more than $10,000 \mathrm{~Hz}$ for the wave response. The instruments should match in electrical or mechanical impedance to minimize undesirable interactions and be properly shielded to maintain a high signal-to-noise ratio.

- The number and location of the transducers (accelerometers, strain gages, etc.) should provide adequate coverage and redundancy for the desired measurement. The use of additional transducers for noise cancellation or control should also be considered.

- The sampling frequency for digitizing data should be greater than two times the highest frequency of significant package response. The digitized data must be filtered to eliminate alias frequencies inherent to the sampling process.

- Low-pass filters used for separating the quasistatic response from the vibration and wave responses must have proper cut-off characteristics and a cut-off frequency sufficiently lower than the lowest vibration frequency of the package but considerably higher than the dominant frequencies of the quasi-static response, so that the filtering process can remove the vibration and wave responses with minimal effects on the quasistatic response. This requirement implies that the filter method can be used to separate the quasi-static response from the wave and vibration responses, only if the lowest vibration frequency of the package is considerably higher than the dominant quasi-static response frequencies. Using a 4th-order Butterworth low-pass filter as an example, Appendix $C$ shows that the package vibration frequency should be greater than 4.1 times quasi-static frequency which is defined as the inverse of the impact duration. When using this filter, the cutoff frequency should be set at about 0.3 times the lowest vibration frequency of the package.

Using a sample acceleration time history measured during a drop test, Appendix $\mathrm{C}$ demonstrates how the peak rigid-body acceleration and the corresponding stress and strain of the test specimen can be obtained by filtering the data. In addition, Appendix $\mathrm{C}$ shows that the rigid-body impact stress and strain are appreciably smaller than the total impact stress and strain, because the wave and vibration effects are significant in this case. Thus Appendix $\mathrm{C}$ confirms the discussion in Subsection 2.1.2 and Appendix A; the rigid-body response is only one component of the total impact response. A review of Appendix $\mathrm{C}$ before the planning of instrumented measurements is highly recommended.

\subsection{Other Measurements}

Section 9 will show that the time history information provided by instrumented measurement of the impact response of a package is extremely useful. However, there are cases where the cost of instrumented measurement may not be justifiable. Packages of very simple design are examples. For these cases, response measurements should still be made but with simpler and less expensive measuring devices or sensors. These devices usually can provide crude (unfortunately, sometimes extremely crude) estimates of the peak value but not the time histories of the response. For example, resettable and non-resettable impact indicators are commercially available to measure peak accelerations ranging from several to thousand g's. Brittle coating can be used to identify an area of high tensile strain. Pressure-sensitive paint which changes color permanently under pressure can be used to obtain peak pressure contours on component interfaces. When using these simple devices, it is essential (as in instrumented measurements) that the devices are properly calibrated and placed at the proper locations for the desired response measurement. 
7. Test Measurements 


\section{TEST RECORD}

Test records should be as complete and accurate as practical. Any deviation from the original test model, test plan and test procedures should be recorded, and the reason or justification for the change should also be given. The possible effects of the change on the test operation and results should be evaluated, and the test procedures revised to accommodate the change.

A complete set of impact test records should include test model design, pre-test inspection reports, pre-test measurements, test conditions, test procedures, test measurements, post-test inspection reports, and post-test measurements.

Records of the test model should contain all engineering drawings and specifications used for the fabrication, assembly, and quality control of the model.

Inspection reports may include results of acceptance tests and of destructive and nondestructive examinations. The records should describe the methods and conditions used for the tests and the examinations and acceptance criteria used for the evaluation of the results.

Photographic records of the examinations should be preserved to support the conclusions.

Records of test conditions should include all conditions that may affect the test results. The conditions usually consist of the impact conditions, the initial environmental conditions, and the initial conditions of the test model. The initial conditions of the test model include initial temperatures, pressures, and damage to the model. Recording the initial damage to the model is essential because a test model is usually reused in several impact tests, either to minimize the number of test models used or to meet the regulatory requirement for the sequence of impact tests. Photographic and video records of the impacting package should contain time and length information.

The records of pre- and post-test measurements should include drawings which identify the specific dimensions measured. Each of the dimensions should have pre- and post-test measurements.

Records of instrumented measurements should include drawings which identify the location and orientation of the transducers. The records should include the calibration results of the transducers and instruments. The recorded outputs of the transducers should contain timing marks for synchronizing the recorded signals. The time and magnitude range settings used for the recording, and other helpful information concerning the recorded measurements should also be recorded on the audio or voice track of the same magnetic tape. 
8. Test Record 


\section{TEST DATA ANALYSIS}

The analysis of test data should achieve three objectives:

- Determine the reliability of the data

- Extract desirable impact information from the test model

- Evaluate the safety performance of the package in terms of the extracted information

Operator errors, instrument malfunctions and inherent instrument imprecisions hurt the reliability of the test data. In every test operation, the test conductor should be vigilant to detect and correct problems which may affect the data reliability. In data analysis, the analyst should use the following methods to detect potential problems:

- Examine individual data to identify unreasonable or unexpected behavior

- Compare similar data to detect dissimilar behavior

- Compare related data to detect uncorrelated or unexplainable behavior

- Repeat the same measurement to determine the size of possible errors or the scatter of data

All unusual behaviors indicate possible problems with the data. If the precise cause and its effect on the data cannot be determined, the suspected data should be discarded as unreliable data. Large scatter of data indicates imprecision of the measurement process and the instability of the response. A greater number of tests or measurements may be required to overcome the problem.

The above-described general methods are essential and applicable to all test measurements and observations. In pre- and post-test measurements and inspections, the reliability of the data or observations should be determined by the inspector in the field when the data are collected. Unreliable data should be discarded and the measurements repeated immediately. In instrumented test measurements, a quick check should be carried out by performing a pre- and post-test calibration of all instruments and displaying the recorded data. These steps will determine whether or not the test should be repeated.

Once the data are deemed reliable, desired information can be extracted from the recorded data. The extraction method varies with the data and the desired information. Analysis of the results of all inspections, examinations, acceptance tests and measurements is beyond the scope of this report. The following discussion is limited to instrumented test measurements.

As described in Subsection 7.2, the output of instrumented measurements is electrical signals which contain noise and other unwanted responses from the instruments and the package. Eliminating the noise and unwanted responses is a major task in analyzing the data. The prerequisite for this task is an understanding of the signal contents and their characteristics. To this end, the signals should be examined in both the time and frequency domains. Responses, like waves which have sharp peaks and a broad frequency range, can be identified easier in the time history of the signals. On the other hand, responses like vibrations and electrical noise from an $\mathrm{AC}$ power source which have distinct frequencies can be identified more clearly in the Fourier spectrum of the signals. Spectrum analyzers are commercially available to facilitate signal analysis in both time and frequency domains. A well-equipped spectrum analyzer can filter, digitize, analyze the input signal and display the results in various formats: time histories, Fourier spectra, power density spectra, correlation spectra, etc..

Once the characteristics of the signal contents are understood, specific methods can be chosen to eliminate the unwanted contents. Filtering can be used to eliminate from the impact test data electrical noise and alias frequencies (resulting from digitization), and to separate package impact responses. Details of these operations are given in Appendix $C$. The filters needed for these operations are also described in Appendix C. 
To understand the instrumented measurements and the impact behavior of the package, display and examine the time histories of all related outputs together. By correlating the responses at different times and locations, the nature of the response can be identified. In general, the response tends to show greater wave effect at earlier times and greater vibration influence at later times after impact. Similarly, the response would appear more like quasi-static deformation at locations nearer than farther from the impact area. Areas of high local vibrations and wave 'effects can also be identified. To illustrate the benefit of the approach, Figures 9-1-9-7 display in groups the drop test results of the Defense High-Level Waste (DHLW) shipping cask from Madsen, Uncapher, Stenberg, and Baynes (1987). The following are possible interpretations of the data present in these figures:

- Figure 9-1 indicates that the stiff bottom ring (or bottom impact limiter) of the cask has caused a high wave/vibration response of the cask during the bottom-end drop

- Figure 9-2 shows that the bottom-end drop was not perfect and has caused a high bending stress in the cask wall near the impact point. However, the axial stress in the cask wall becomes more uniform, and the bending stress decreases with increasing distance, from the impact point. The same figure also indicates the presence of permanent compressive deformations in the cask wall.

- A comparison of the results from Figures 9-1 and 9-3 indicates that the notch impact limiter at the cask top is able to lengthen the duration of a top-end drop relative to the bottom-end drop and to increase the significance of the quasi-static deformation response relative to the vibrational response.

- Figure 9-4 shows that during the top-end drop, the closure lid experienced two impacts including the main impact between the unyielding target and the entire cask, and a subsequent impact between the canister and the closure lid caused by the rebound of the cask.

- Figure 9-5 shows that the closure bolts experienced tension during a puncture test on the closure lid. This result seems to suggest the presence of a prying action on the closure bolts, which can be caused by the bending of the closure lid under the puncture load.

- Figures 9-6 and 9-7 clearly show the prominence of vibrations in the cask response to the side drop.

The test results of the DHLW cask are more difficult to measure and interpret than others, because the stiff impact limiters excite greater wave and vibration responses than quasi-static response. However, even in this difficult case, the above study of the results has demonstrated that . instrumented measurements can provide meaningful and useful information and understanding of the impact behavior of the package, which can help improve and justify the design.

The procedures and requirements for data analysis should be given in the test procedures, which are discussed in the next section of this report. 


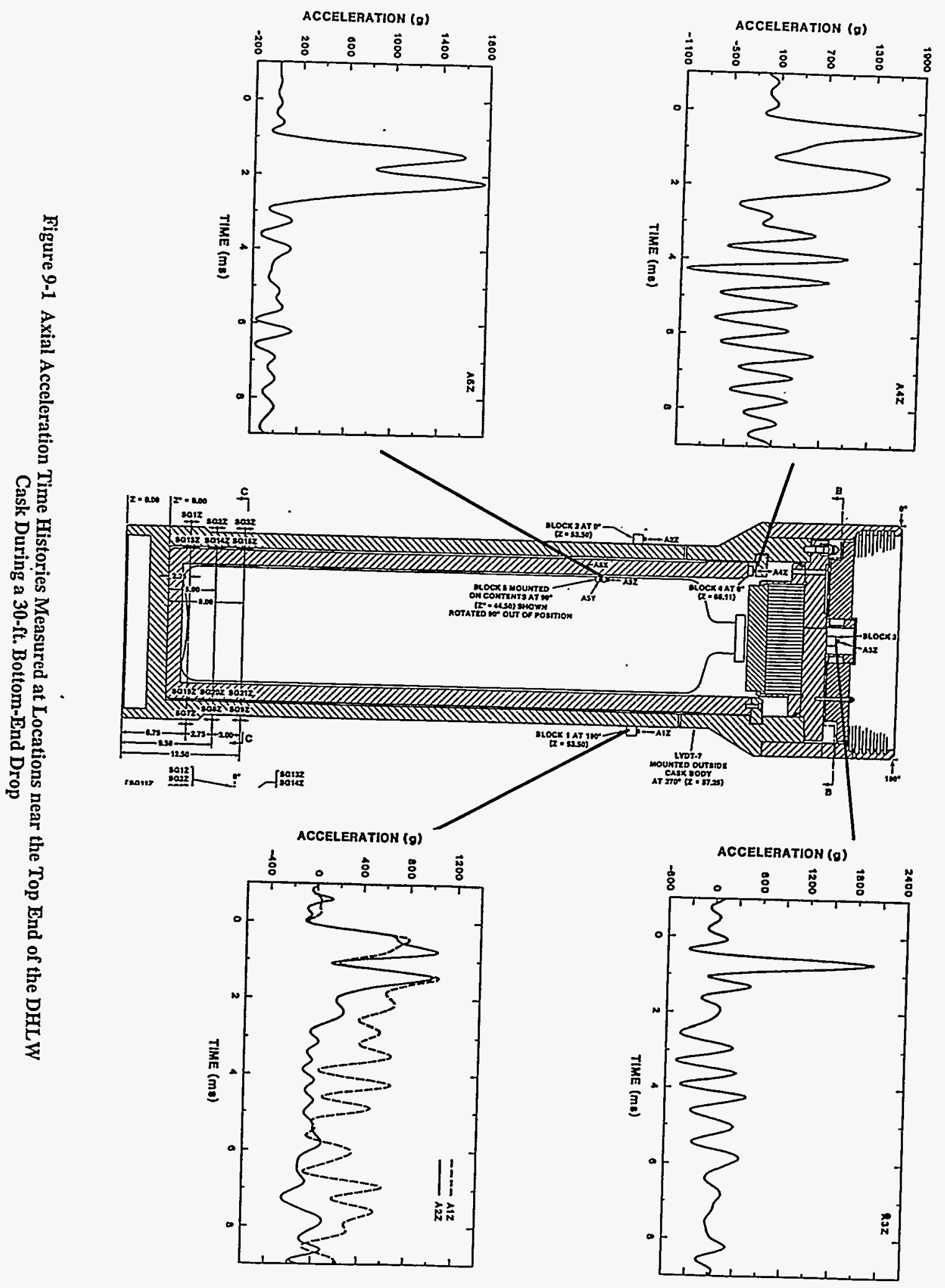



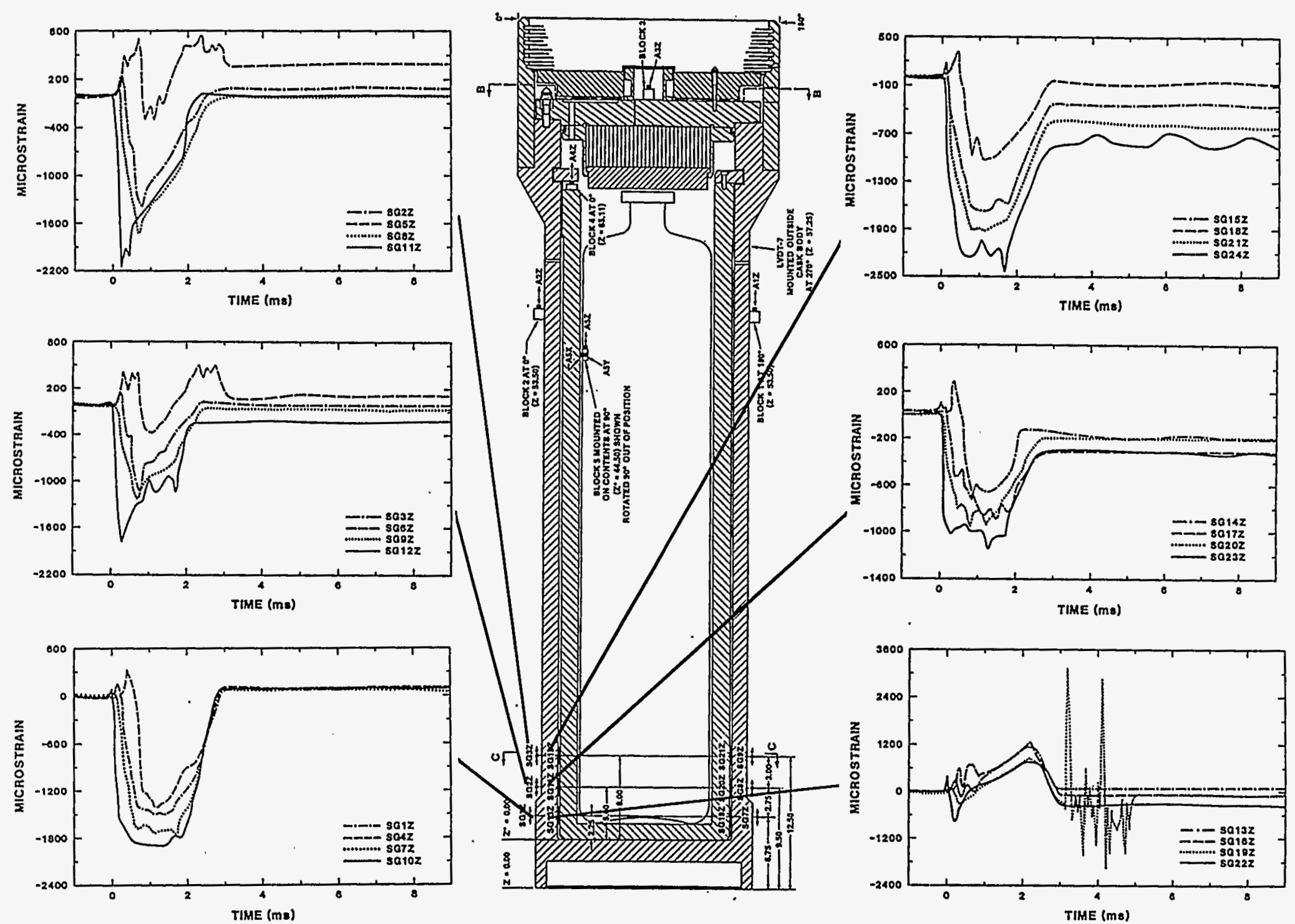

Figure 9-2 Axial Surface Stain Histories Measured at Locations on the Outer and Inner Surfaces of the DHLW Cask Druing a 30-ft. Bottom-End Drop 


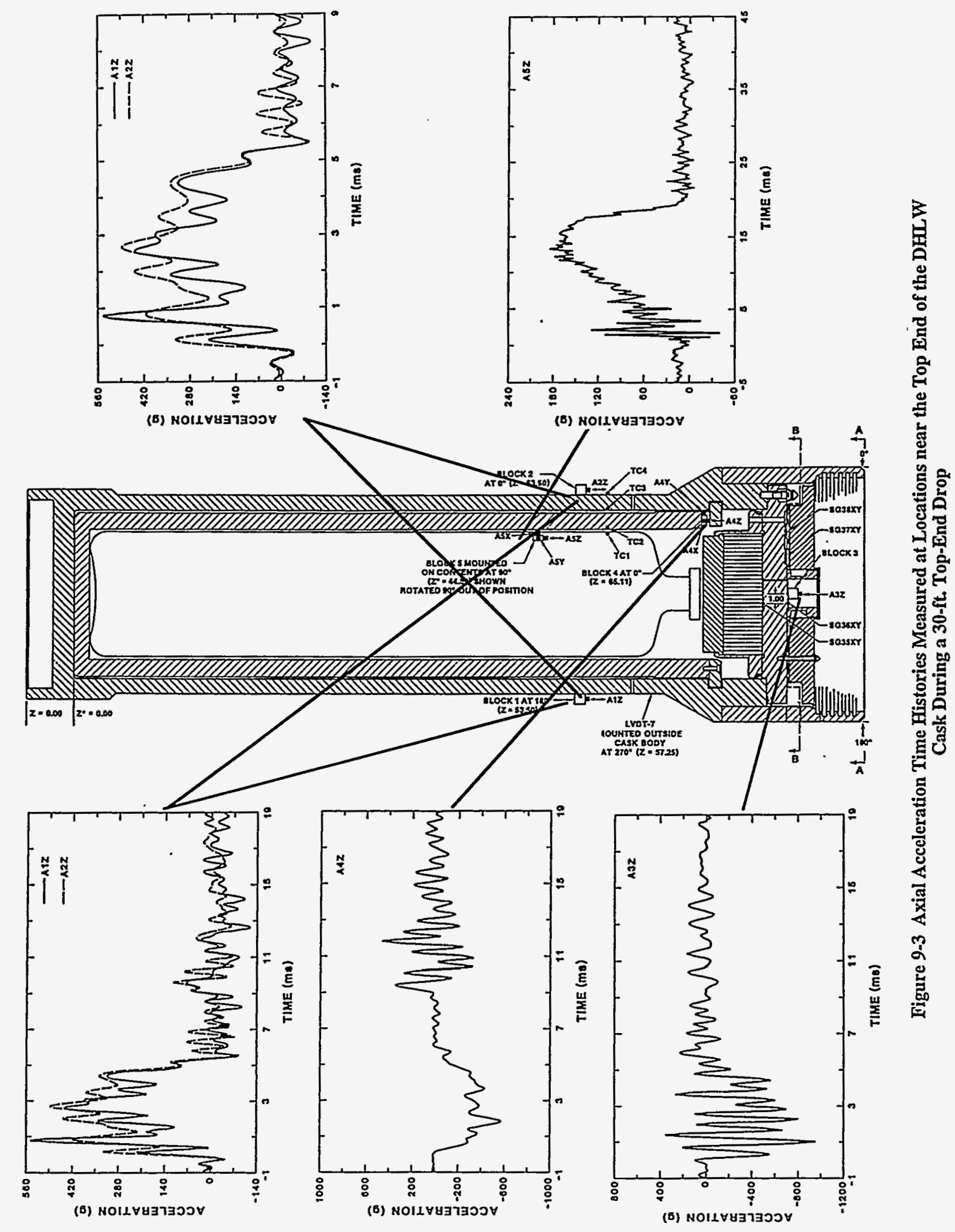



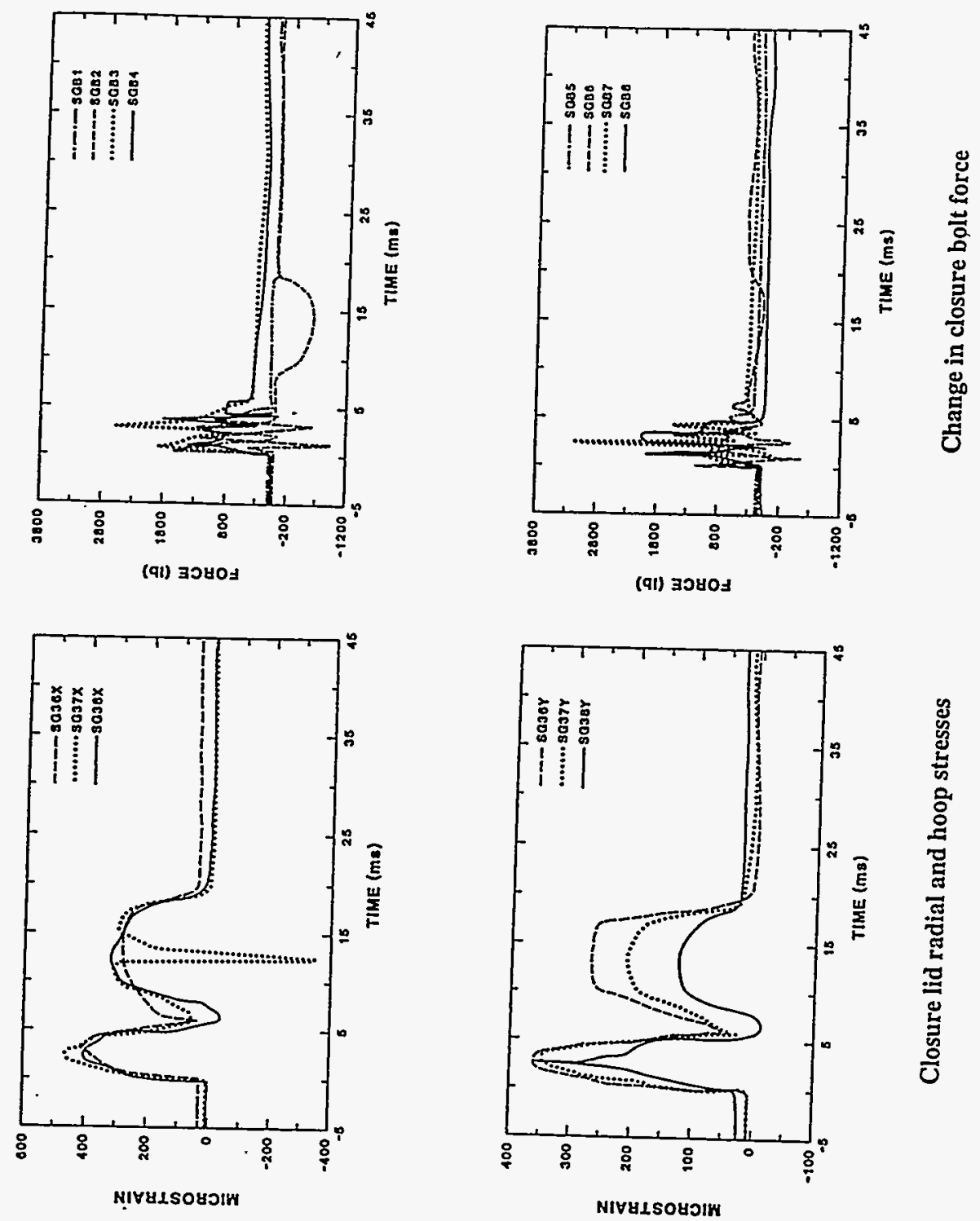

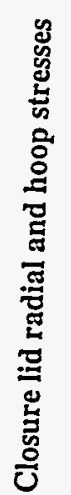
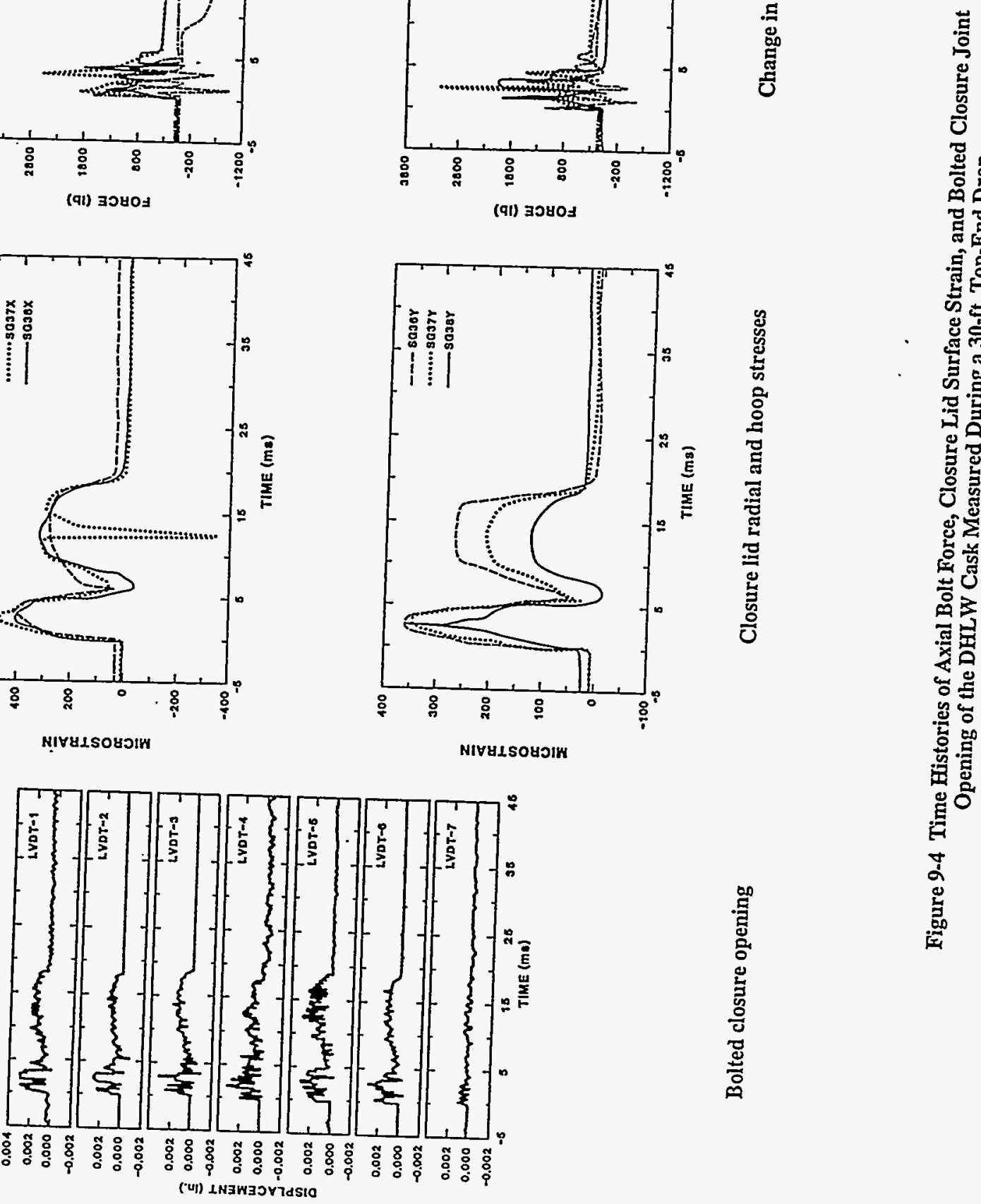

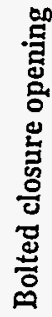



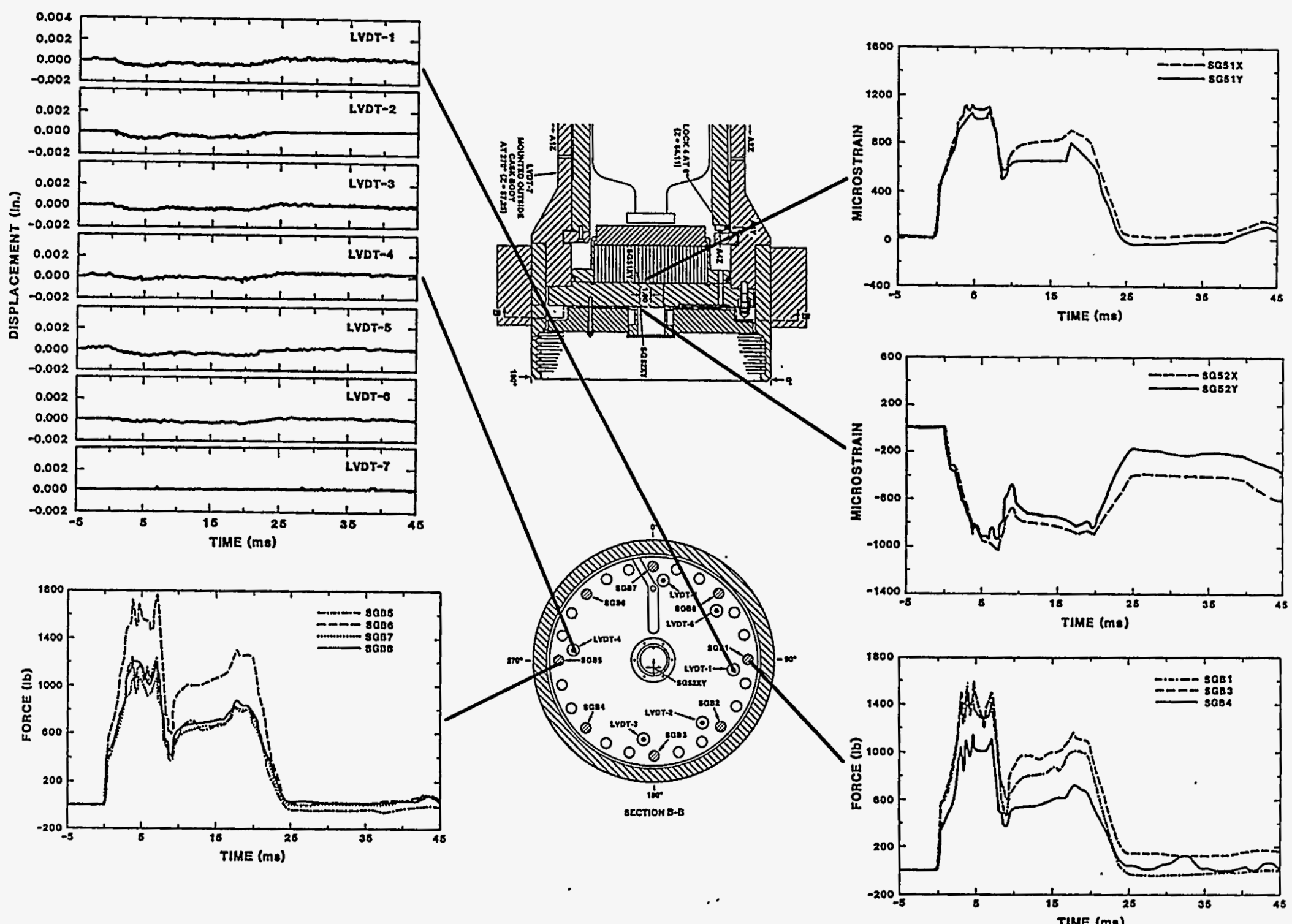

Figure 9-5 Time Histories of Axial Bolt Force, Closure Lid Surface Strain, and Bolted Closure Joint Opening of the DHLW Cask Measured During a 40-in. Puncture Impact at the Center of the Closure Lid 

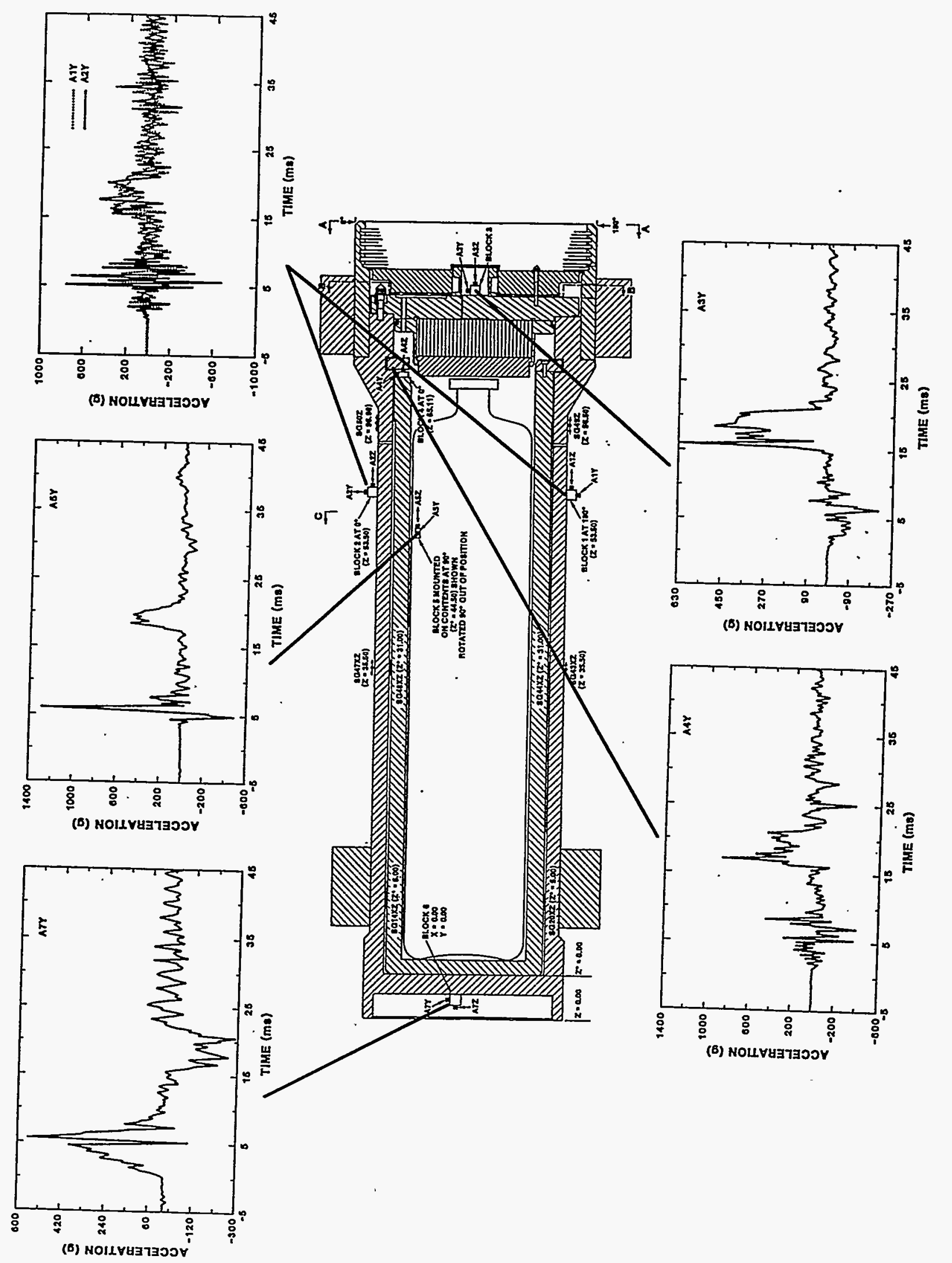

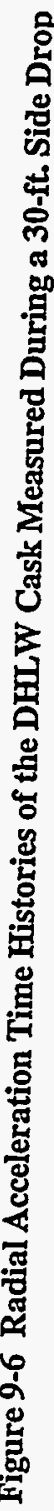




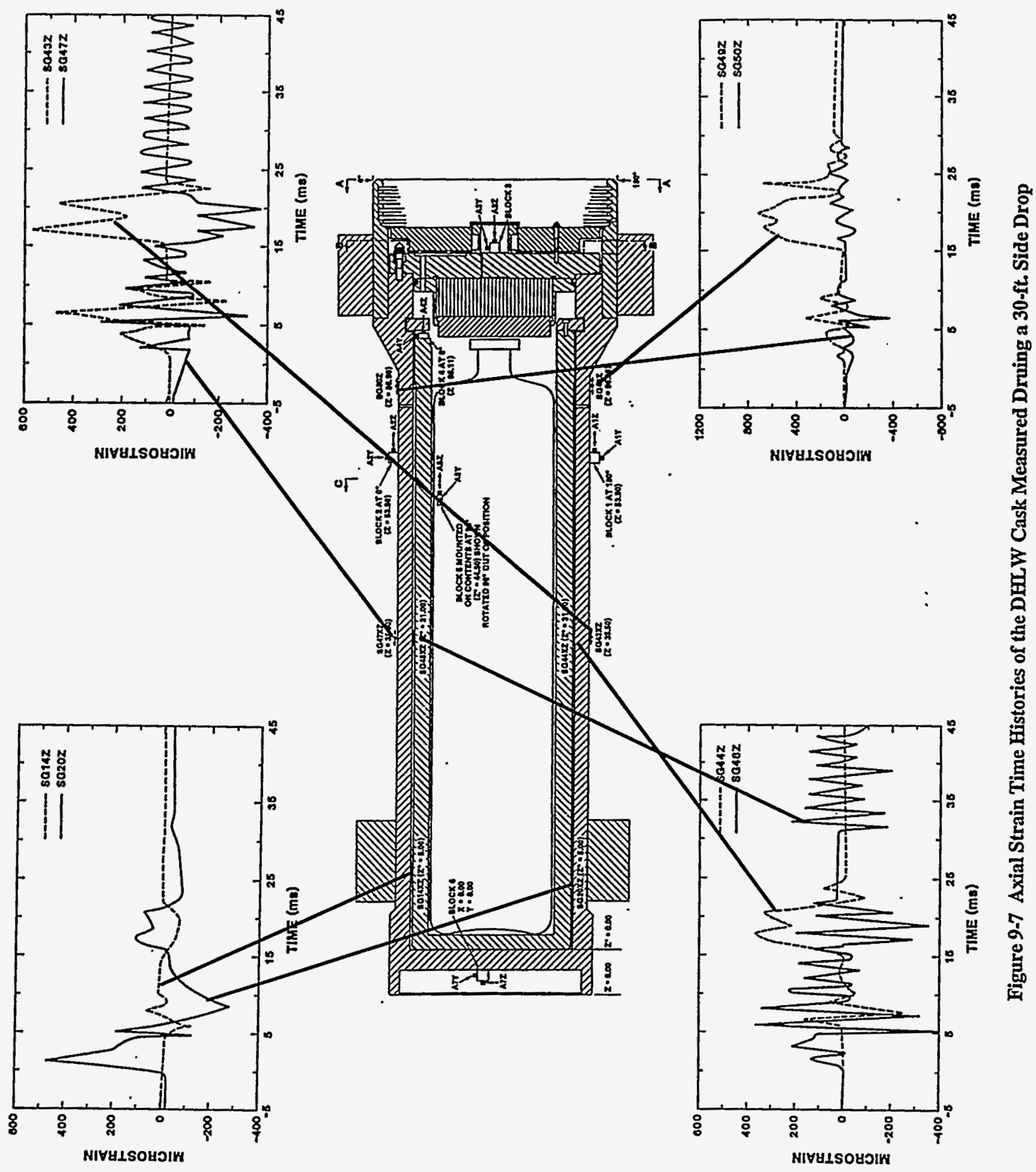


9. Data Analysis

This page left blank intentionally. 


\section{TEST PROCEDURE}

The test plan should be documented and supplemented by detailed test procedures. A good test plan is meaningless unless it is properly implemented. The tools to accomplish this goal are the test procedures. The test procedures provide the test operators with precise instructions. In addition, during the preparation of the test procedures, the test planner has an opportunity to mentally exercise his test plan and detect possible deficiencies in the plan. Briefly, the test procedures serve the following functions:

- To avoid errors or loss of data due to negligence and disorganization

- To set standards and control quality

- To ensure safety

The test procedures should include step-by-step instructions for every major step:

- Fabrication or preparation of the test model

- QA inspection of the test model

- Acceptance test of the test model

- Calibration of test equipment and instruments
- Installation of the transducers on the test model

- Setup of equipment and instruments

- Pre-test measurements

- Setup of the impact test

- Recording of instrumented measurements

- Post-test inspection of the test model

- Post-test measurements

- Post-test acceptance tests

- Storage of test records

- Reduction or analysis of test data

The test procedures should sufficiently detail the operations to ensure the quality. The detailed information also help document the test operations and record the steps taken to correctly implement the operations. The test procedures should be very useful in preparing the test report, which is discussed in the next section. 
10. Test Procedure 


\section{TEST REPORT}

An impact test program for a shipping package may generate a large number of test data and records. To simplify and facilitate the reporting of the data, each test operation may have its own report. All reports provide valuable detailed information and should be used to prepare the final or summary report for the test program. The final report should contain mainly information to support the objectives of the impact test program.

The final report of a compliance test program should clearly show how the test results demonstrate the compliance of the package with the regulatory requirements. In essence, the final report needs to answer the following questions:

- What are the acceptance criteria for the indirect demonstration of compliance? (What are the test measurements/observations and corresponding limits to be used for the demonstration?)

- Why are the acceptance criteria equivalent to the regulatory requirements?

- Is the test model prototypical? If not prototypical, what are the differences between the test model and the actual package? Why would the differences not affect the validity of the test results?

- What are the test conditions? Why are they selected? Have they included all potentially critical conditions?
- What test measurements and observations have been made, and by what methods? How accurate are the test results?

- What industrial standards and quality assurance measures have been used in every operation of the test program to ensure the reliability of the test results?

- How are the test measurements/observations compared to the limits set by the equivalent acceptance criteria? How do they demonstrate the compliance of the package with the regulatory requirements?

- What other information have the test measurements and observations revealed concerning the impact behavior of the shipping package? Are they consistent with the assumptions made in the selection of the test model, test conditions, instruments, and in the establishment of the equivalent acceptance criteria? Are there indications of imminent failure or unstable behavior which would cause concerns about the reliability of the test results and the available safety margin of the package design?

The test report needs to present the test conclusions and all supporting evidences to demonstrate that the conclusions are reasonable, reliable, and adequate for the intended purpose of the test program. 
11. Test Report 


\section{SUMMARY AND CONCLUSION}

A package for the transportation of radioactive material is very different from an ordinary shipping package. Because of the radioactivity of the contents, the package must meet specific regulatory requirements to ensure public health and safety. An impact test program to demonstrate the compliance of the radioactive materials package with the regulation is not as straightforward and simple as a normal package acceptance test. For instance, unless the radioactive contents are used, the radiological consequences of an impact cannot be measured directly and must rely on interpretations and assessments of test results. This unique situation with the radioactive materials package has several implications: (1) the test program can not be standardized; the test plan has to vary with the package design and the method used to demonstrate package compliance, (2) an adequate understanding is required of the impact behaviors of all safety-related components of the package, and (3) test measurements, in addition to the apparent distortion of the package, are needed to demonstrate the adequacy of the test plan and conclusion. In response to these needs, this report has identified the guidelines for an impact test program for radioactive material shipping packages. The report has also discussed the reasons for the guidelines, the possible difficulties in meeting these guidelines, and the available methods to overcome the difficulties.

Planning an impact test program for the radioactive material packages involves two major initial tasks.

Determine approach to demonstrate compliance. The first task is to determine the approach used to demonstrate the package compliance with the regulation. Two approaches have been used in the past; they can be identified as the compliance and the benchmark test programs. In a compliance test program, the impact test results are directly compared to a set of acceptance criteria to show compliance with the regulation. In a benchmark test program, the test results are used to validate an impact analysis method or model, and the validated method or model is then used to analytically generate the necessary results to compare with the acceptance criteria. In both approaches, a set of acceptance criteria is required. The adopted criteria have to be shown to meet or exceed the regulatory standards on the containment, shielding, and subcriticality of the radioactive contents of the package. This equivalence is usually established using appropriate containment, shielding and criticality analyses of the package. The adopted test approach and the equivalent acceptance criteria determine the basic test measurements to be made in the test program.

\section{Select Test Conditions}

The second initial task in planning an impact test program is selecting the environmental and impact test conditions. The regulation requires that the selection contains the conditions most damaging to the safety performance of the package. The proper selection of the condition depends on the design, impact behavior, and possible failure modes of the safety-related components of the package. The adequacy of the selection will require confirmation or justification which may be obtainable from visual evidence and measured data collected during the impact test program. Justifying the test conditions will determine other test measurements to be made in the test program, in addition to those needed for meeting the acceptance criteria.

A complete impact test plan should specify detailed requirements for the design and fabrication of the test model, the pre- and post-test inspections, measurements, and acceptance tests, the test setup and conditions, the acquisition and analysis of test measurements, the storage of test records, and the contents of test reports. The details should include the methods/procedures, the standards/criteria, and the QA practices to follow in the various operations.

The test model used for a compliance test should be prototypical, which means it is identical to the actual package in design, fabrication, and QA procedures and standards. Other models may be used only with substantial justification. The use of 
scale models will be difficult to justify for the following purposes: (1) to measure the rate of leakage through a bolted closure joint of a package; (2) to demonstrate structural integrity of a package prone to brittle and buckling failures; (3) to simulate the impact response of a package made of strain-rate-sensitive materials; (4) to simulate drop tests which produce peak impact (g) loads not significantly higher than the package weight; or (5) to simulate the effects of contents heat on the impact response of a package. The possible effects of design and fabrication details on the scale model behavior should be investigated. The details include welds, joints, the coarse microstructures of honeycomb and concrete, etc. Ideally, the applicability of the scaling laws for a package or package component is proven by comparing results obtained using scale models of more than one size. Past drop test studies have shown that scale models smaller than one-quarter scale usually produce unsatisfactory results.

The purpose of pre-test inspections, examinations (NDE), and acceptance tests is to certify the quality of the test model. These pre-test procedures should be carried out in accordance with the acceptance standards and the requirement of the design code used for the design and construction of the package.

The post-test inspections and examinations are used to determine the extent of the impact damage. Unless fracture and crack growth are failure modes of concern, the post-test inspections can be accomplished without using the sophisticated NDE methods such as RT or UT for detecting flaws and cracks. A thorough visual inspection, a comprehensive dimension survey, and a limited number of destructive examinations will suffice. In packages where the damage can be caused by wave and vibration responses as well as the quasi-static response, the inspection should cover the entire package. The inspection should include not only the vicinity of the impact area and weak structural members where the quasistatic response tend to inflict the most damage, but all package component interfaces and structural joints where the wave and vibration responses can cause damage.

In a benchmark test program, instrumented timehistory measurement of the acceleration, stress/strain, or displacement of the impact response of the safety-related components of the package should be taken and compared with analysis results. Likewise, instrumented measurements should also be taken in a compliance test program for packages with significant wave and vibration responses. In all cases, sufficient acceleration measurements should be taken to help analyze all non-trivial components of the rigid-body acceleration of the package.

Instruments used to measure response should have adequate sensitivity, linearity, frequency response, and signal-to-noise ratio. They should be properly matched in impedance, and protected from noise to minimize the effect of impedance and noise on the measurements. Anti-aliasing filtering, using low-pass filters of adequate frequency response, should be used to rid an analog signal of high-frequency noise before sampling the signal for analog-to-digital conversion. The frequency response of filters used to separate wave, vibration and quasi-static impact responses should also be properly chosen based on the frequency contents of the responses.

In addition to reporting the test methods and results, the test report should include sufficient experimental evidences and analytical justifications to support the conclusion. 


\section{BIBLIOGRAPHY}

Allen, G. C. Jr., et al. 1987. "Development of a Large Unyielding Target for Package Tests." The 8th International Symposium on the Packaging and Transportation of Radioactive Materials (PATRAM '86), Davos, Switzerland, June. Proceedings published by IAEA, Vienna, Austria, Vol. 2, pp. 457-464, January.

Baker, W. E. 1960. "Modeling of Large Transient Elastic and Plastic Deformations of Structures Subjected to Blast Loading." J. Appl. Mech., Transactions of the ASME, Vol. 27, pp. 521-527, September.

Barnfield, J. H. and P. J. Donelan. 1985. "Demonstration Drop Test." Paper No. 5 in The Resistance to Impact of Spent Magnox Fuel Transport Flasks. Proceedings from a seminar held from 30 Apr. to 1 May at the Institution of Electrical Engineers, Savoy Place, published by the Institution of Mechanical Engineers, London.

Beauchamp, K. G. 1973. Signal Processing.Using Analog and Digital Technique..John Wiley \& Sons, New York.

Biggs, J. M. 1964. Introduction to Structural Dynamic. McGraw Hill, New York.

Bouche, R. R. 1979. Calibration of Shock and Vibration Measuring Transducers. Shock \& Vibration Monograph \#SVM-11. The Shock and Vibration Information Center, U.S. Naval Research Laboratory, Washington, D.C.

Bray, D. E. and R. K. Stenley. 1989. Nondestructive Evaluation (A Tool for Design, Manufacturing, and Service), McGraw Hill, New York.

Bridgman, P. M. 1931. Dimensional Analysis. Yale University Press, New Haven, CT.

Broek, D. 1982. Elementary Engineering Fracture Mechanics, 3rd ed. Martinus Nijhoff, Publisher, Boston.

Brown, K. 1959. Package Design Engineering. John Wiley \& Sons, New York.

Buckingham, E. 1914. "On Physically Similar Systems; Illustration of the Use of Dimensional Equations." Physical Review, Series 2, Vol. 4, pp. 345-76.

Buckingham, E. 1915. "Model Experiments and Forms of Empirical Equations." Transactions of the ASME, Vol. 37, p. 263.

Bumpus, S. E., et al. 1989. "Benchmarking of the Computer Code and the Thirty-Foot Side Drop Analysis for the Shipping port RPV/NST Package." The 9th International Symposium on the Packaging and Transportation of Radioactive Materials (PATRAM '89). Washington, D.C., June. Proceedings published by Oak Ridge National Laboratory, Oak Ridge, Tennessee, Vol. II, pp. 979-986.

Chun, R. C., T. Lo, G. C. Mok, and M C. Witte. 1991. "SCANS Theory Manual Vol. 3, Lead Slump in Impact Analysis and Verification of Impact Analysis." U.S. Nuclear Regulatory Commission Report NUREG/CR-4554, Vol. 3, Rev. 1.

Dally, J. W. and W. F. Riley. 1991. Experimental Stress Analysis. 3rd ed.., McGraw Hill, New York.

De Silva, C. W. 1983. Dynamic Testing and Seismic Qualification Practice. Lexington Books, Lexington, MA. 
Bibliography

Dimentberg, M. F., K. V. Frolov, and A. I. Menyailov. 1991. Vibroacoustical Diagnostics for Machines and Structures. John Wiley \& Sons, New York.

Donelan, P. J. and A. R. Dowling. 1985. "The Use of Scale Models in Impact Testing." Paper No. 4 in The Resistance to Impact of Spent Magnox Fuel Transport Flasks. Proceedings from a seminar held from 30 Apr. to 1 May 1985 at the Institution of Electrical Engineers, Savoy. Place, published by the Institution of Mechanical Engineers, London.

Drucker, D. C. 1962. "Plasticity and Viscoelasticity, Basic Concepts." Handbook of Engineering Mechanics, chapter 46, W. Flugge, ed., reissue 1980, McGraw Hill, New York.

Duffey, T. A. 1971. "Scaling Laws for Fuel Capsules Subjected to Blast, Impact and Thermal Loading." The 1971 Inter-Society Energy Conversion Engineering Conference, Society of Automotive Engineers, Inc.

Edwin, D. J. 1984. Modal Testing: Theory and Practice. John Wiley \& Sons, New York.

Eller, E. E. and R. M. Whittier. 1988. "Piezoelectric and Piezoresistive Transducers." Chapter 12 in Shock and Vibration Handbook, C. M. Harris, ed., 3rd ed.., McGraw Hill, New York.

Ezra, A. A. and F. A. Penning. 1962. "Development of Scaling Laws for Explosive Forming."

Experimental Mechanics (Journal of the Society for Experimental Stress Analysis). Vol. 2, pp. 234-249, August.

Gerhard, M. A., D. J. Trummer, G. L. Johnson, and G. C. Mok. 1991. "SCANS (Shipping Cask ANalysis System)-A Microcomputer-Based Analysis System for Shipping Cask Design Review. Vol. 1-User's Manual to Version 2a." U.S. Nuclear Regulatory Commission Report NUREG/CR-4554, Vol. 1, Rev. 1.

Glass, R. E. 1988. "Structural Code Benchmarking Data Report." Sandia National Laboratory Report \#88-3362, Albuquerque, NM, May.

Goldman, L. H., et al. 1988. "Shippingport Reactor Pressure Vessel/Neutron Shield Tank Package Benchmark Model Drop Test Report." Westinghouse Hanford Company Report SSDP-0049, August.

Gonzales, A. 1987. "Target Effects on Package Response: An Experimental and Analytical Evaluation." Sandia National Laboratory Report SAND86-2275, Albuquerque, NM, May.

Gonzales, A., et al. 1986. "Target Hardness Comparisons with the IAEA Unyielding Target." The 8th International Symposium on the Packaging and Transportation of Radioactive Materials (PATRAM '86), Vol. 2, pp. 545-551.

Goodier, J. N. 1950. "Dimensional Analysis." Appendix II in the Handbook of Experimental Stress Analysis, M. Hetenyi, ed., 4th printing 1960.

Goodier, J. N. and W. T. Thompson. 1944. "Application of Similarity Principles to Structural Models." Tech. Note 993, National Advisory Committee for Aeronautics.

Gregory, P. C. and S. A. Porter. 1989. "Design and Testing of TRUPACT-II." The 9th International Symposium on the Packaging and Transportation of Radioactive Materials (PATRAM '89), Vol. I, pp. 99-106.

Halmshaw, R. 1991. Non-Destructive Testing. 2nd ed., Edward Arnold, London.

Harris, C. M. 1988. "Measurement Techniques." Chapter 15 in Shock and Vibration Handbook, C. M. Harris, ed., 3rd ed., McGraw Hill, New York. 
Holt, P. J. 1985. "Impact of Magnox Irradiated Fuel Transport Flasks into Rock and Concrete." Paper No. 9 in The Resistance to Impact of Spent Magnox Fuel Transport Flasks, a seminar held from 30 Apr. to 1 May at the Institution of Electrical Engineers, Savoy Place, published by the Institution of Mechanical Engineers, London.

Hudson, D. E. 1961. "Scale-Model Principles." Shock and Vibration Handbook, Vol. 2, chapter 27, C. M. Harris and C. E. Crede, eds., McGraw Hill, New York.

Huerta, M. 1978. "Analysis, Scale Modeling, and Full-Scale Test of a Railcar and Spent-Nuclear-Fuel Shipping System in High-Velocity Impacts Against a Rigid Barrier." Sandia National Laboratory Report SAND77-0270, Albuquerque, NM., April.

Huerta, M. 1981. "Analysis, Scale Modeling, and Full-Scale Test of a Railcar and Spent-Nuclear-Fuel Shipping Cask in a High-Velocity Impact Against a Rigid Barrier." Sandia National Laboratory Report SAND78-0458, Albuquerque, NM., June.

Huerta, M. and R. Yoshimura. 1976. "Adequacy of Scale Modeling of Large Casks." IAEA International Seminar on the Design, Construction and Testing of Packaging for the Safe Transport of Radioactive Materials, Vienna, Austria, August.

Johnson, B. G., ed. 1976. Guide to Stability Design Criteria for Metal Structures. Column Research Council of Engineering Foundation, John Wiley \& Sons, New York.

Joseph, B. J. 1983. "Design and Testing of Scale Model Transport Systems." The 7th International Symposium on the Packaging and Transportation of Radioactive Materials (PATRAM '83). New Orleans, Louisiana, May. Proceedings published by Oak Ridge National Laboratory, Oak Ridge, Tennessee, Vol. 2, pp. 1219-1225, December.

Kelly, R. D. and G. Richmon. 1969. Principles and Techniques of Shock Data Analysis. Shock \& Vibration, Shock and Vibration Monograph SVM-5. The Shock and Vibration Information Center, U.S. Naval Research Laboratory, Washington, D.C.

Kienholz, D. A. and Allen Bradley. 1987. "30-ft. Free Drop Tests of a Quarter-Scale Model 2000 Transport Package." GE Nuclear Energy Division Report NEDO-31581, San Jose, CA. August.

Langhaar, H. L. 1951. Dimensional Analysis and Theory of Models. John Wiley and Sons, New York. Reprint 1980 with minor corrections, Robert E. Krieger Publishing Co., Inc., Huntington, NY.

Lee, E. H. 1962. "Viscoelasticity." Handbook of Engineering Mechanics, chapter 53, W. Flugge, ed., reissue 1980, McGraw Hill, New York.

Madsen, M. M., et al. 1987. "Testing the Half-Scale Model of the Defense High Level Waste Transportation Cask." Sandia National Laboratory Report SAND 86-1131.

Magrab, E. B. and O. A. Shinaishin. 1975. Vibration Testing-Instrumentation and Data Analysis. ASME Publication AMD-Vol. 12, Am. Soc. of Mech. Engineers, New York.

Maji, A. K., S. Donald, and K. Cone. 1990. "Testing of Impact Limiters for Transportation Cask Design." Mechanical Properties of Porous and Cellular Material. Proceedings from the Materials Research Society Symposium, Vol. 207, K. Sieradzki, D. J. Green, and L. J. Gibson, eds., Material Research Society, Pittsburgh, PA.

Marguerre, K. 1962. "Elasticity, Basic Concepts." Handbook of Engineering Mechanics, chapter 33, W. Flugge, ed., reissue 1980, McGraw Hill, New York. 
Neilsen, M. K., et al. 1989. "Development of a Phenomenological Constitutive Model for Polyurethane Foams." The 9th International Symposium on the Packaging and Transportation of Radioactive Materials (PATRAM ‘89), Vol. I, pp. 79-86.

Nelson, T. A. and R. C. Chun. 1987. "Method for Impact Analysis of Shipping Containers." U.S. Nuclear Regulatory Commission Report NUREG/CR-3966, Washington, D.C.

Nolan, D. J., A. G. Eggers, and P. Shih. 1989. "Design, Analysis, and Testing of Wood Filled Impact Limiters." The 9th International Symposium on the Packaging and Transportation of Radioactive Materials (PATRAM '89), Washington, D.C., Vol. I, pp. 44-54, June.

Ott, H. W. 1976. Noise Reduction Techniques in Electronic Systems. John Wiley \& Sons, New York.

Ramboz, J. D., M. R. Werbyn, and R. W. Lally. 1988. "Calibration of Pickups." Chapter 18 in Shock and Vibration Handbook, C. M. Harris, ed., 3rd ed., McGraw Hill, New York.

Robinson, R. A., et al. 1976. "Use of Scale Models to Assess Structural Response of Nuclear. Shipping Containers Under Accident Conditions." IAEA International Seminar on the Design, Construction and Testing of Packaging for the Safe Transport of Radioactive Materials, Vienna, Austria, August.

Smith, J. D. 1989. Vibration Measurement and Analysis. Butterworths, London.

Soper, W. G. 1961. "Dynamic Similitude for Lead." J. Appl. Mech., Vol. 50, pp. 132-133, March.

Soper, W. G. 1964. "Dynamic Modeling with Similar Materials."

Soper, W. G. 1967. "Scale Modeling." International Science and Technology, pp. 60-69, February.

Soper, W. G. and R. C. Dove. 1961. "Data Presentation for Cushioning Materials." Paper No. 27-4, 17th Annual Technical Conference, Society of Plastic Engineers, Inc., January.

Soper, W. G. and R. C. Dove. 1962. "Similitude in Package Cushioning." J. Appl. Mech., Transactions of the ASME, Vol. 20, pp. 263-266, June.

Thomlinson, D. C., P. J. Donelan, and R. Pryer. 1985. "Instrumentation for the Train Crash Demonstration." Paper No. 19 in The Resistance to Impact of Spent Magnox Fuel Transport Flasks. Proceedings from a seminar held from 30 Apr. to 1 May 1985 at the Institution of Electrical Engineers, Savoy Place, published by the Institution of Mechanical Engineers, London.

Uncapher, W. L., R. A. May, and J. D. Miller. 1992. "Basic Impact Limiter Study.” Sandia National Laboratory Report SAND90-2405.

U.S. Code of Federal Regulations. Title 10, Part 71. 1978. "Packaging of Radioactive Material for Transport and Transportation of Radioactive Material under Certain Conditions," as revised in Federal Register, Vol. 48, no. 165, August.

VanSant, J. H., T. F. Chen, and L. E. Fisher. 1992. "Feasibility Studies for the Controlled CruiseAltitude Drop, and Aircraft Crash Tests." Lawrence Livermore National Laboratory Report UCRL-ID112582.

Walter, P. L. and R. Rodeman. 1979. "Characterization of Digital Filters Used in Sandia Laboratories Test Data Analysis Division.” Sandia National Laboratory Report SAND79-1230.

Warrant, M. M. and B. J. Joseph. 1987. "Test Data Report for Quarter Scale NuPac 125-B Rail Cask Model." Sandia National Laboratory Report SAND85-2129, December. 
Wilson, E. J. 1988. "Strain-Gage Instrumentation." Chapter 17 in Shock and Vibration Handbook, C. M. Harris, ed., 3rd ed., McGraw Hill, New York.

Window, A. L., G. S. Holister, ed. 1982. Strain Gage Technology. Applied Science Publishers, London and New Jersey.

Witte, M. C. 1986. "Comparison of Static and Dynamic Finite Element Methods for Shipping Container Impact Analyses." The 8th International Symposium on the Packaging and Transportation of Radioactive Materials (PATRAM '86), Vol. 2, pp. 659-667.

Yoshimura, H. R., et al. 1989. "1/3 Scale Model Testing Program." The 9th International Symposium on the Packaging and Transportation of Radioactive Materials (PATRAM '89), Vol: III, pp. 1678-1685.

Yoshimura, H. R., et al. 1989. "Force and Moment Reconstruction for a Nuclear Transportation Cask Using Sum of Weighted Accelerations and Deconvolution Theory." The 9th International Symposium on the Packaging and Transportation of Radioactive Materials (PATRAM '89), Vol. III, pp. 1499-1506.

Zimmer, A. and M. Koploy. 1991. "Alternate Approaches to Verifying the Structural Adequacy of the Defense High-Level Waste Shipping Cask." General Atomics Report GA-A20783. 
Bibliography

This page left blank intentionally. 


\section{APPENDIX A: IMPACT RESPONSE AND PACKAGE DESIGN}

An impact can excite three dynamic responses of a package structure: the wave, the vibration, and the quasi-static-deformation responses. All three responses contribute to the package response. Depending on the package design and the drop orientation, any one or a combination of these responses can cause damage to the package structure. Most package designs have soft impact limiters. The impact response of these packages is dominated by the quasi-static deformation response. In packages with hard impact limiters, the wave and vibration responses are also significant. Recognizing the significant responses and their characteristics is key in planning an impact test program.

Figure A-1 offers a simple demonstration of the unique characteristics and the interrelationship among the three dynamic responses. The four acceleration time histories in the upper portion of the figure were artificially generated for the demonstration. The first curve from the top of Fig. A-1 depicts a typical acceleration time history of the wave response. It represents a longitudinal compressional wave undergoing multiple reflections between the fixed and the free end of a cylinder. The individual acceleration pulse of short duration in the time history reflects the sudden jump of particle velocity across the wave front. Each pulse in the history indicates a passage of the wave front through the monitoring location. A higher amplitude and shorter duration of the acceleration pulse indicate a sharper wave front. Lengthening of the duration and decay of the amplitude of the impulse with increasing time is due to the dispersion, scattering, and dissipation of the wave energy. The second curve from the top of Fig. A-1 depicts a typical acceleration time history of the vibration response. It indicates a structure vibrating at one of its natural frequencies. The time history is a sine curve of varying amplitude. Increasing the amplitude at the beginning represents the initial buildup of the vibration after the application of an external excitation. The subsequent decrease of the amplitude reflects the dissipation of the vibration after the removal of the excitation. The sine curve has a frequency near the vibration frequency of the structure. The third curve from the top of Fig. A-1 is a typical acceleration time history of the quasi-static deformation response. It shows the change of the acceleration of a rigid body when it falls onto a stationary deformable object (the impact limiter in the case of a package). The rise of the acceleration at the beginning of the impact corresponds to the deceleration of the impacting rigid body due to the resistance of the deformable object. The subsequent drop-off of the acceleration coincides with the rebound of the rigid body. The stiffness of the deformable object and the impact energy of the impacting rigid body determine the total impact duration. The fourth curve from the top of Figure A-1 was created by superimposing the preceding three time histories of the individual dynamic responses. The composite curve represents the impact acceleration time history in a package, where the quasi-static response dominates. Below this artificial time history in Figure A-1 is an actual acceleration time history selected from the test results of the Defense High-Level Waste (DHLW) shipping cask. The similarity of the actual and the artificial time histories demonstrates the presence of the three impact responses in the package impact response. Section 9 of this report presented additional time histories from the DHLW cask test program, some of which exhibit significant wave and vibration responses.

To identify the test requirements for individual impact responses, a discussion of the cause and characteristics of each response follows.

Of the three impact responses, the wave appears first at the start of the impact. It is caused by the sudden change of velocity (or momentum) of the package material at the impact area. The wave is a messenger or distributor; it carries the sudden momentum change from the impact area to other parts of the package and eventually distributes the impact energy to the 
entire package. The wave has a finite magnitude and travels at a finite speed which is determined by the wave motion, the local stiffness and mass properties of the package structure. In a package with a hard impact limiter, the stress wave can have a very prominent front (i.e., a step change in the velocity, and stress/strain of the material at the wave front) when the wave is first generated at the impact area. However, as the wave travels into the package, the sharpness of the front dulls quickly due to the scattering and dispersion of the wave energy. The causes for the scattering and dispersion are reflections and refractions of the waves at the material. and geometric boundaries of the package structure. The wave front, however, may still be able to traverse the package structure several times before it dissipates into an unnoticeable disturbance. As this process is taking place, the wave energy is transferred to the vibration and quasi-static-deformation responses of the package. To measure the acceleration, stress, and strain caused by the wave front, an instrument of very-high-frequency limit is needed. The wave response can be significant in a package with hard impact limiters and few. features to effect appreciable dispersion and scattering of the wave. In such a package, the wave energy can converge into a concentrated area to cause spallation damage or local buckling of the package structure. A spallation damage of the closure bolts can happen in the end-on impact of a cylindrical package without limiter. The lack of an impact limiter can cause a large-amplitude compressional wave to be generated at the impact end of the package. If the wave travels with little attenuation, it will be reflected at the other end as a strong tensile wave. If the closure lid is located at this end, the tensile wave can inundate the closure lid with sufficient momentum to break the closure bolts by tension. Wave damages can occur in areas away from the impact area and tend to appear near package component boundaries and discontinuities where the wave is reflected.

The vibration response is the result of the package structure responding to the impact in the natural vibration modes of the package. A package has an infinite number of vibration modes. However, only the modes with the frequency closest to the excitation frequency, the modes with the lowest frequencies, and the modes with a mode shape closest to the spatial distribution of the excitation are sensitive to the excitation and show appreciable response. Therefore, if the frequency and mode shape of these modes are known, the vibration response can be identified in the impact test measurements. Once the dominant vibration modes are recognized, the distribution of the response in the package can also be estimated and the critically stressed area identified, because the vibration response is distributed according to the mode shape. This information in turn determines, for the impact test of a package, the critical locations to be inspected, the frequency range to be used in the selection of instruments and equipment for data collection and analysis. Effective analytical and experimental methods exist to determine the vibration frequencies and mode shapes of a package. Large vibration response can cause instant permanent deformation or rupture similar to the wave response. Small vibration response can only cause (high-cycle) fatigue failure which would not appear until the package experiences a sufficient number of cycles of the vibration. In an impact test, such fatigue damage is unlikely, due to insufficient cycles of vibration.

The quasi-static deformation response is the result of the whole-body or rigid-body motion of the package. The rigid-body acceleration at the center of gravity of the package determines the response. The total impact force of the quasi-static response is determined by the rigidbody acceleration and the total mass of the package. The distribution of quasi-static deformation and stress in the package is determined by the distribution of acceleration and mass in the package. In the simplest case of an end-on impact of a cylindrical package, where the rigid-body acceleration is uniformly distributed and collinear with the impact force, the distribution of the quasi-static impact deformation in the package is similar to the deformation caused by the weight of a package when it is supported at the impact area. In the 
case of an oblique impact, the similarity does not exist because of the presence of the rotational acceleration. In the most general case, the rigid-body acceleration has six components including three rotational and three translational components. The measurement of the rigidbody acceleration in an impact test should include all six components. Damages caused by the quasi-static impact deformation usually appear near the impact area. Damages can also appear. in a weak structural section away from the impact area if the section is under a disproportionately heavy inertial load. The quasi-static deformation needs time to develop and dominate the package response. Therefore, the impact duration can provide some indication of the significance of the quasi-static response. If the impact duration is shorter than the longest vibration period (of the lowest vibration mode) of the package, the vibration . response is likely to be significant. The impact duration should also be used to estimate the frequency range requirement of instruments for the measurement and data analysis of the quasistatic response.

Based on the preceding discussions, the test requirements of the various impact responses can be identified and summarized as follows.

The test model should adequately simulate the design features affecting the dominant impact response of the package. The wave and vibration responses need a more precise distribution of mass and stiffness properties in the package than the quasi-static response. They also require a better reproduction of the mechanical behavior and impedance at the component joints of the package. For example, a welded joint should not be replaced with a bolted joint. An effective way to determine the adequacy of a model for wave and vibration responses is to perform a modal survey of the test model. If the model can reproduce the dominant vibration frequencies of the package, the model is adequate.

The critical test condition varies with the impact responses. The selection of the test conditions for the quasi-static response can be based on the impact force or acceleration and the static deformation of the package; the greater the impact force, the greater the quasi-static response. The selection of the test conditions for the wave and vibration should also consider the wave and vibration behavior of the package.

The post-test inspection should cover locations where damages caused by the dominant responses tend to occur. The area near the impact point and the weak structural components of the package should be inspected for damages caused by the quasi-static response. If the wave and vibration responses are significant, the inspection should be expanded to include component interfaces and structural joints.

The frequency response of the measurement instruments should be adequate for measuring the impact response. Of the three responses, the wave response has the highest frequencies. The frequencies of the vibration response are lower, and the frequencies of the quasi-static response are the lowest. An instrument for measuring all three impact responses requires a widefrequency range extending from $0 \mathrm{~Hz}$ up to a high-frequency limit determined by the wave and vibration responses. 
Appendix A: Impact Response and Package Design

Artificial acceleration time histories

Typical stress wave response

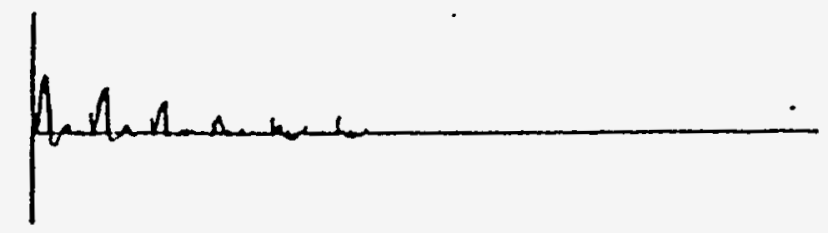

Typical vibration response

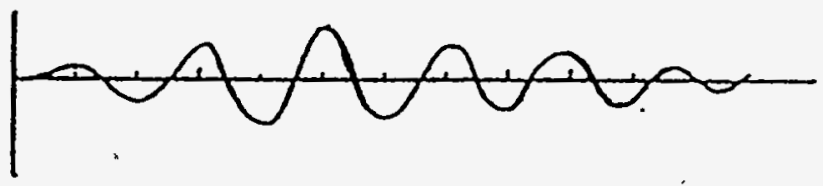

Typical quasi-static response

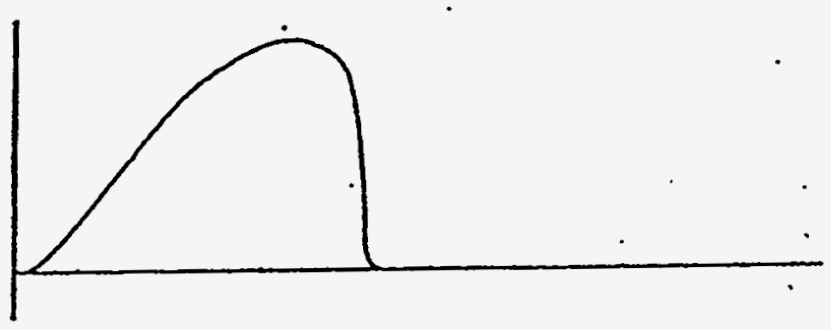

Combined response

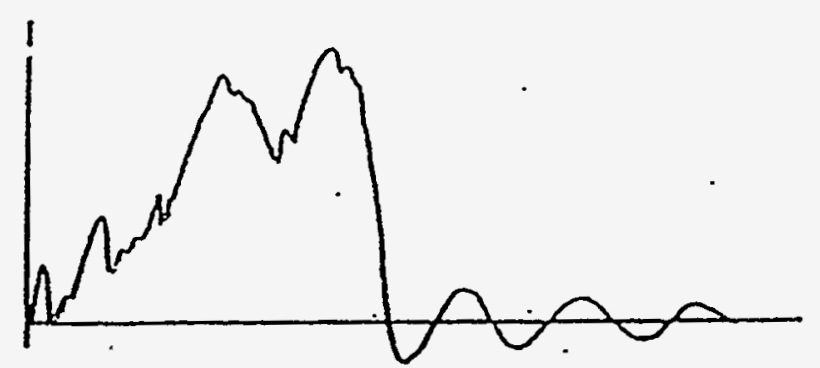

Actual package acceleration time history

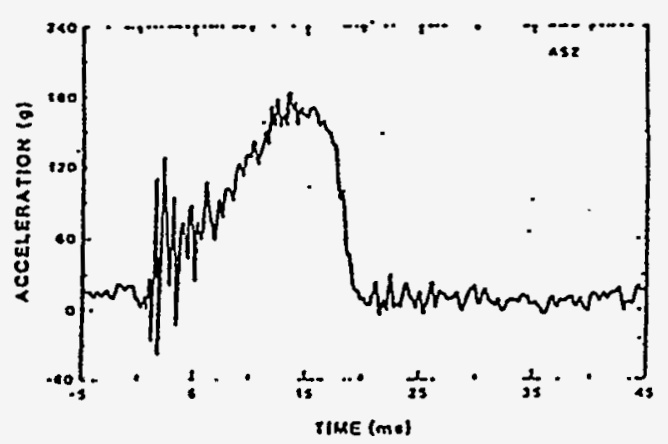

Figure A-1 Acceleration time history of impact responses. 


\section{APPENDIX B: SCALE-MODEL IMPACT TESTING}

\section{B.1 Introduction}

This appendix details the basis and background for the recommendations given in the main report on the use of scale models for drop testing transport packages containing radioactive materials. The appendix does not directly address the simulation of the safety performance of the packages, which is measured in terms of leakage rates and other radiological limits. Instead, this appendix describes the simulation of structural or mechanical response of the packages, which includes deformations, forces, etc. It is assumed that the structural response can be properly translated into the safety performance.

Specifically, the appendix discusses: (1) the past use of scale-model drop testing for transport packages, (2) general scaling laws for impact motion, stress and deformation, (3) theoretical and practical difficulties in the implementation of the scaling laws, and (4) general guidelines for scalemodel impact testing.

\section{B.2 Past Use of Scale-Model Drop Test}

Scale models are used because they are often less expensive to produce and test. This statement is particularly true when the test is conducted to obtain performance parameters affected only by a few properties of the prototype. In this case, only a few details need to be simulated, and the test model will cost much less than the prototype.

Past drop tests using a scale model can be grouped by objective into two categories: (1) tests to obtain key data for validating an analysis or an analytical tool which is then used to justify the design of a package [Bumpus et al. 1989, Goldman et al. 1988, Nolan et al. 1989, Robinson et al. 1976]; (2) tests to measure structural performance or to demonstrate structural integrity of a package [Huerta 1978 and 1981, Warrant and Joseph 1987]. No attempt appears to have been made to use scale models to directly demonstrate the compliance of a package with the regulatory safety-performance requirements. Leak tests on scale models with bolted closures have been conducted following a drop test on those models. However, there exists no indication that the results have been used and accepted as the complete evidence of the compliance of the packages with the regulatory requirement for containment.

The requirements and guidelines developed in this appendix are directly applicable to the first two categories of the above-mentioned scale model tests. Tests of the third category (compliance tests) require either the scaling of the safety performance parameters or the correlation of the structural and safety performance parameters. These two tasks are not always possible, because the underlying phenomena for some of the safety performance parameters like leakage rate through the space between two contacting surfaces are not sufficiently understood. Scale-model tests for demonstrating safety performance require special treatments beyond the scope of this appendix. In many cases, a scale-model test may not offer sufficient economic advantage to offset the many technical uncertainties. Thus, a full-scale compliance test may remain a preferred choice, as it did for TRUPACT-II [Gregory 1989].

\section{B.3 Scale Model and Scaling Laws}

The mechanical response and failure modes of a structure vary with the applied loading, the geometry, material, and temperature of the structure. For example, a compressive load tends to produce buckling failures while a tensile load is more inclined to cause rupture. A slender structure can buckle easier than a stocky structure. A structural component with a notch or with a long internal flaw can fail easier by brittle fracture than a structure without. A material has a greater tendency to deform and to creep at higher temperatures and to fracture at lower temperatures. Therefore, a scale model for predicting the structural response and failure mode must properly simulate the loading, geometry, material, and temperature of the structure. Some radioactive materials have high decay heat; therefore, the simulation of temperature in the scale model is desirable. (The 
absorption of impact energy in the package can also cause the temperature in the package to rise. However, for the low impact velocity of the regulatory drop test, this effect is highly localized to the area near the impact area and the effect on the total package is negligible.) Unfortunately, the simulation of heat transfer using a scale model is generally considered an impossible task. Therefore, the present discussion omits the simulation of temperatures. It is assumed that the scale model and the prototype have the same temperature distribution or the effects of different temperature distributions can be properly accounted for in the interpretation of the scale model test results.

The standard procedure to develop a scale model and the supporting scaling laws are well established. The necessary processes are as follows:

- Specify the behaviors to be simulated with the scale model

- Understand the physical phenomena causing these behaviors

- Identify the physical parameters controlling these phenomena

- Develop the scaling laws for these controlling parameters by imposing the conditions: (1) The parameters are consistent with each other in dimension; (2) The parameters are similarly related in the scale model as in the prototype.

The last process is commonly known as the dimension analysis [Buckingham 1914 and 1915, Bridgman 1931, Langhaar 1951, Goodier and Thompson 1944, Goodier 1950, Hudson 1961]. It consists of two steps:

- Form dimensionless groups of the controlling parameters

- Define the scaling laws in terms of the dimensionless parameter groups

The first step is mathematical and straightforward. The second step depends on the model designer's experience and understanding of the phenomenon to be simulated. The second step is intuitive unless all the governing equations of the phenomenon are known. Then the governing dimensionless groups can be precisely identified from the equations. This precise information usually provides a greater freedom in the design of the scale model; distorted models without complete geometric similarity are possible. Unfortunately, governing equations are known only for a few simple situations.

The use of specific equations is not possible here, because the present discussion focuses on a general package whose design and governing equations are undefined. Thus, the scaling laws must be derived from the general principles which govern the impact response.

In general, to have similar mechanical response, the prototype and the scale model must have:

- Geometric similarity

- Kinematic similarity

- Dynamic similarity

- Gravitational similarity

- Material similarity

These conditions assure that the geometry, the material, the load, the constraint, the impact response, and the governing physical laws are all similar in the prototype and the scale model.

\section{B.3.1 Geometric Similarity}

A complete geometric similarity requires that all geometry-related quantities be scaled using a single scale factor, $\mathrm{s}_{\mathrm{L}}$. A scale factor is defined here as the ratio of a quantity in the scale model to the corresponding quantity in the prototype:

$\mathrm{s}_{\mathrm{L}}=\frac{L_{\text {scalemodel }}}{\mathrm{L}_{\text {prototype }}}$

where $L$ is a typical length.

The geometry-related quantities to be scaled with the same factor are:

- geometric dimensions

- locations of impact point 
- locations of center of gravity

- locations of measurement sensor

- displacements and deformations

Large deformations, which are likely to occur in a drop test, will appreciably alter the package geometry. Therefore, the geometric similarity will require that the same scale factor be used to scale the geometry and deformation:

$$
\mathrm{s}_{\mathrm{u}}=\frac{\mathrm{u}_{\text {scalemodel }}}{\mathrm{u}_{\text {prototype }}}=\mathrm{s}_{\mathrm{L}}
$$

where $u$ denotes a typical displacement or deformation.

The same requirement may also be necessary for small deformations, if the load and constraint patterns and the structural response of the package are very sensitive to deformations. Some examples are: the buckling of a structural component in the package, and the closing of a small gap between two neighboring components.

The use of the same scale factor for deformation and geometry means identical strains in the scale model and the prototype:

$s_{\varepsilon}=\frac{\varepsilon_{\text {scale model }}}{\varepsilon_{\text {prototype }}}=1$

where $\varepsilon$ is a typical strain.

This result would restrict the choice of materials for the scale model.

\section{B.3.2 Kinematic Similarity}

This similarity requires that the motions of the prototype and the scale model be similar at homologous locations and homologous times. This requirement implies that the displacements $(u)$, velocities ( $v$ ) and accelerations (a) of the prototype and the scale model are related by only two scale factors, namely, a time factor $\left(s_{t}\right)$ and a displacement factor $\left(s_{u}\right)$;

$$
\mathrm{s}_{\mathrm{u}}=\frac{\mathrm{u}_{\text {scale model }}}{\mathrm{u}_{\text {prototype }}}
$$

$$
s_{t}=\frac{t_{\text {scale model }}}{t_{\text {prototype }}}
$$

$$
s_{v}=\frac{v_{\text {scale model }}}{v_{\text {prototype }}}=\frac{s_{u}}{s_{t}}
$$

$s_{a}=\frac{a_{\text {scale model }}}{a_{\text {prototype }}}=\frac{s_{u}}{s_{t}^{2}}$

Invoking the requirement of geometric similarity, $s_{u}=s_{L}$, Eqs. (6) and (7) are rewritten in terms of SL;

$s_{v}=\frac{s_{L}}{s_{t}}$

$s_{\mathrm{a}}=\frac{s_{\mathrm{L}}}{s_{\mathrm{t}}^{2}}$

\section{B.3.3 Dynamic Similarity}

This similarity requires corresponding force $(F)$, mass (M), and acceleration (a) to obey Newton's laws in the prototype and in the scale model, i.e.,

$\left(\frac{\mathrm{F}}{\mathrm{Ma}}\right)_{\text {scale model }}=\left(\frac{\mathrm{F}}{\mathrm{Ma}}\right)_{\text {prototype }}$

The equation can be expressed in terms of scale factors:

$\mathrm{s}_{\mathrm{F}}=\mathrm{s}_{\mathrm{M}} \mathrm{s}_{\mathrm{a}}$

where $s_{F}, s_{M}$, and $s_{a}$ are scale factors for the force, mass, and acceleration, respectively. 


\section{B.3.4 Gravitational Similarity (load similarity)}

The similarity of force or load between the scale model and the prototype requires that the direction of all forces be unchanged and the magnitude be scaled using a single scale factor. Therefore, the ratio between any two forces will be unchanged from the prototype to the scale model, i.e.,

$$
\left(\frac{F_{g}}{F_{i}}\right)_{\text {scale model }}=\left(\frac{F_{g}}{F_{i}}\right)_{\text {prototype }}
$$

where $F_{g}$ and $F_{i}$ represent the gravitational force and the impact inertial force, respectively. For a material particle of a unit volume, the gravitational and impact forces are equal to $\rho g$ and $\rho v^{2} \pi$, respectively, where $\rho$ is the mass density of the material; $g$ is the gravitational acceleration; $v$ is the impact velocity and 1 is the limiter crush; $v^{2} /$ is a measure of the peak deceleration of the impact. Thus Eq. (12) becomes:

$$
\left(\frac{\lg }{v^{2}}\right)_{\text {scale model }}=\left(\frac{\lg }{v^{2}}\right)_{\text {prototype }}
$$

or in terms of scaling factors,

$$
s_{\mathrm{u}} s_{\mathrm{g}}=\mathrm{s}_{\mathrm{v}}^{2}
$$

The scale factor for the limiter crush is the scale factor for all displacements and deformations $\left(s_{\mathfrak{u}}\right)$.

\section{B.3.5 Material Similarity}

For a homogeneous material, the mass property is completely defined by its mass density, $\rho$. The stiffness property, however, is more complicated and is defined by a set of constitutive laws involving stress, strain, and sometimes strain rate. The constitutive laws or the stress-strain relation of many materials including metallic and foam materials can be expressed in the following general form [Marguerre 1962, Drucker 1962, Neilson 1989]:

$$
\mathrm{G}\left(N \sigma / \sigma_{0}, \mathrm{E \varepsilon} / \sigma_{0}, \mathrm{H} \dot{\varepsilon} / \sigma_{0}, \mathrm{~K} / \sigma_{0}\right)
$$

where $\sigma, \varepsilon$, and $\varepsilon$ are the stress, strain, and strain rate in the material, respectively; $\sigma_{0}$ is a characteristic or reference stress; $\mathrm{N}, \mathrm{E}, \mathrm{H}$, and $\mathrm{K}$ are material constants defined later in this subsection. The symbol G( ) represents a function of the dimensionless variable groups:

$\mathrm{N} \sigma / \sigma_{0}, \mathrm{E} \varepsilon / \sigma_{0}, \mathrm{H} \dot{\varepsilon} / \sigma_{0}$, and $\mathrm{K} / \sigma_{0}$. The function can be in any mathematical form; it will be a linear function for an elastic material and a non-linear function for an elastic, plastic material. For material of the same behavior, $G$ will have the same mathematical form. Thus, if materials of the same behavior are used for the scale model and the prototype, the constitutive laws of the materials will be similar if the value of each of all the dimensionless variable groups in Eq $\dot{q}$.(15) is kept unchanged. In addition, the force similarity requires that the ratios $\sigma / \sigma_{0}$, and $\sigma_{0} / \sigma_{i}$ remain unchanged between the scale model and the prototype, where $\sigma_{i}$ is the impact inertial stress, i.e., $\sigma_{\mathrm{i}}=\mathrm{F}_{\mathrm{i}} / \mathrm{A}$, and $\mathrm{A}$ is the stress area. The resulting conditions for the similarity of the constitutive laws of the material are given in the following equations:

$$
\left(\frac{1 \mathrm{~A} \sigma}{\rho v^{2}}\right)_{\text {scale model }}=\left(\frac{1 \mathrm{~A} \sigma}{\rho v^{2}}\right)_{\text {prototype }}
$$

$$
(\mathrm{N})_{\text {scale model }}=(\mathrm{N})_{\text {prototype }}
$$

$$
\left(\frac{\mathrm{K}}{\sigma}\right)_{\text {scale model }}=\left(\frac{\mathrm{K}}{\sigma}\right)_{\text {prototype }}
$$

$$
\left(\frac{E \varepsilon}{\sigma}\right)_{\text {scale model }}=\left(\frac{E \varepsilon}{\sigma}\right)_{\text {prototype }}
$$




$$
\left(\frac{\mathrm{H} \dot{\varepsilon}}{\sigma}\right)_{\text {scale model }}=\left(\frac{\dot{\mathrm{H}}}{\sigma}\right)_{\text {prototype }}
$$

Imposing the geometric-similarity requirements that the same scale factor be used for the geometrical dimensions and the displacements $\left(\mathrm{s}_{\mathrm{L}}\right.$ $=\mathrm{s}_{\mathrm{u}}$ ), and that the strains in the scale model and the prototype are equal $\left(s_{\varepsilon}=1\right)$, Eqs.(16)-(20) can be simplified and rewritten in terms of scaling factors;

$$
\begin{aligned}
& s_{L}^{3} s_{\sigma}=s_{\rho} s_{v}^{2} \\
& s_{N}=1 \\
& s_{K}=s_{\sigma} \\
& s_{E}=s_{\sigma}
\end{aligned}
$$

and

$$
s_{\mathrm{H}}=\mathrm{s}_{\sigma} \mathrm{s}_{\mathrm{t}}
$$

Equations (21) through (25) are the scaling laws for the material mass density, $\rho$ and the material constants, $\mathrm{N}, \mathrm{E}, \mathrm{H}$, and $\mathrm{K}$. The equations show that the scaling of the mass and stiffness properties are related.

For scale-model components where the mass property can be ignored, Eqs.(12) and (14) need not be satisfied. Instead, they are replaced by an equivalent set of equations relating the applied force, $F$, to the material deformation stress, $A \sigma$ :

$$
\left(\frac{A \sigma}{F}\right)_{\text {scale model }}=\left(\frac{A \sigma}{F}\right)_{\text {prototype }}
$$

OF
$s_{\sigma}=\frac{s_{F}}{s_{L}^{2}}$

Equations 22-25 show the scaling of the material constants, $\mathrm{N}, \mathrm{E}, \mathrm{H}$, and $\mathrm{K}$ to be determined by the dimension of the constants. The dimensionless material constant, $N$, which is like the Poisson's ratio of an elastic material (called the "ratio" constant in this report), must be kept unchanged between the prototype and the scale model. The "modulus" material constant, E, like Young's modulus of an elastic material, and the "stress" material constant, $\mathrm{K}$, like the yield stress of an elastic, plastic material have the dimension of stress. They, therefore, are scaled in the same way as the stress. Similarly, the "viscosity" material constant, $\mathrm{H}$, representing the material's dependence on the strain rate, must be scaled as a product of the stress and time. The foregoing discussion and equations only demonstrate how each type of the material constants be scaled. They do not address such questions as "What are the constants? "How many constants are needed to completely characterize the material stiffness property?" and "How many are needed to simulate in a scale model?" The answer to these questions depends on the material and the need of the scale model. For example, if a homogeneous, isotropic material is known to behave linearelastically during a drop test, the stiffness property of the material can be completely characterized using two material constants such as Young's modulus and Poisson's ratio. However, if the impact response is known to depend mainly on Young's modulus, only the modulus will need to be simulated. If the impact generates permanent deformations, then the yield stress and the entire stress-strain curve above yielding should be simulated. As for rate-dependent materials, the strain rate effect is not sufficiently understood to define a general constitutive law like those of the elastic- and rate-independent plastic material. The rate dependence can be more complicated than that presented in Eq.(15). The stress may depend not only on strain rate but also on stress rate, etc. [Lee 1962]. Thus, in general, the scaling of ratedependent material is impossible. 
If identical materials are used for the prototype and the scale model, the stress must be kept unchanged for a rate-independent material and both the stress and time must be unchanged for rate-dependent materials. This result can be seen from Eq.(23)-(25) by setting the scale factor of the material constants equal to 1 . As shown in the next section, this requirement of keeping both the stress and time unchanged for a rate-dependent material make it impossible to include the simulation of rate effects in such a scale model.

\section{B.4 Development of Scale Models}

\section{B.4.1 Prerequisites}

The preceding sections (B.3.1-B.3.5) have specified the most general scaling laws or requirements for impact testing. Not all these requirements can be met in a single scale model because of fundamental and practical difficulties, some of which the following section will identify. Fortunately, in many practical situations, not all general requirements need to be met, because part of the impact response and material behavior can be considered insignificant for the situation and the scaling of these behaviors can be ignored. Thus, it is imperative that the developer of a scale model have a general understanding or expectation of the structural and material behaviors of the package to be impact tested. To assist this effort, Appendix A of this report describes the causes, characteristics and modeling requirements of various structural and material behaviors which can occur in a package under impact.

Appendix A divides the impact response into wave, vibration, and rigid-body (or quasi-static) responses. Knowing which of the three responses dominates the impact response of the package to be tested is essential to the design of a scale model. Each of the responses depends on different properties of the structure. The properties should be modeled precisely if the response is to be simulated in the model. The rigid-body response, being a steady-state, global response, depends mainly on the total mass of the package and on the force-deformation property of the package near the impact area. Geometry details having no bearing on these properties will be unnecessary to model. On the other hand, the wave response is a transient and localized phenomenon. Its magnitude is affected greatly by the precise distribution of the mass and stiffness of the package as well as the geometry of the reflecting boundaries. Consequently, a model with much greater material and geometry details will be needed for the wave than for the rigid-body response. The vibration response is a global response which also depends on local conditions at boundaries of material and geometry. Similar to the response, the failure modes can also be divided into local and global ones with the local modes requiring greater model details.

Knowing the dominant response in a package is also important to the impact analysis of a package. A traditional method for the analysis is the quasi-static method [Brown 1959, Nelson and Chun 1987]. Implicit in the assumption of the method is that the wave and vibration responses can be ignored, and only the rigid-body response is analyzed. However, when the wave and vibration responses are significant, the quasi-static solution will be different from the actual response [Witte 1986] and should be adjusted using a "dynamic load or amplification factor" [Bigg 1964]. If a scale model is used to verify the dynamic amplification factor, the model must be designed to also simulate the wave and vibration responses.

The unyielding target used for the regulatory drop test is specified as "a flat and horizontal surface of such a character that any increase in its resistance to displacement or deformation upon impact by the test specimen would not significantly increase the damage of the specimen." [Allen et al. 1987, Gonzales 1986 and 1987.] This requirement implies that the target has no significant wave and vibration responses and behaves mainly like a massive rigid body. Accordingly, the target must have a hard surface, a large mass to minimize surface displacement, and a cubic or cylindrical form to disperse the wave and minimize the wave reverberation and resulting vibration. The target for a scale model should meet these requirements, if the prototype target cannot be used. 


\section{B.4.2 Valid Scale Models and Their Limitations}

Since all the parameters used to specify the scale model must have consistent dimensions and there are only three independent dimensions for dynamic mechanical response, i.e., the length (L), time $(t)$, and mass $(M)$ or force $(F)$, there can exist, at the most, three independent scale factors whose value can be arbitrarily chosen. The number of these independent scale factors can be further reduced by theoretical and practical constraints and render the scale model testing impossible. This section shows some of these constraints, and how the constraints can be overcome by ignoring some of the secondary effects in a drop test.

The difficulty in the design of scale model can be shown by examining the requirements for gravitational similarity and material similarity, i.e., Eq.(14) and Eqs.(21)-(25). Since it is difficult to change the gravitational acceleration in a drop test, the gravitational acceleration is usually kept unchanged for the scale model test, that is:

$$
\mathrm{s}_{\mathrm{g}}=1
$$

Thus, the condition for gravitational similarity, Eq.(14) can be satisfied only if

$$
s_{L}=s_{v}^{2}
$$

Since $s_{v}=s_{L} / s_{t}$ is based on dimension consideration, Eq. (29) requires $s_{L}=s_{t} .5$. Therefore, the scale factors for time and length can no longer be independently selected.

Similarly, if identical materials are used in the model and the prototype, the scale factors for the mass density, $\rho$, and all the material constants, $N$, $\mathrm{E}, \mathrm{K}$, and $\mathrm{H}$ will be equal to 1 in Eqs.(21) through (25), resulting in the following conditions:

$$
\begin{aligned}
& s_{\sigma}=1 \\
& s_{t}=1
\end{aligned}
$$

and
$s_{L}^{3}=s_{v}^{2}$

Eq. (32) contradicts Eq. (29). Therefore, if the same material, whether rate sensitive or not, is used in the prototype and the scale model, the gravity effect must be ignored unless the gravitational acceleration can be changed. Eq.(32) also contradicts Eq. (31) unless $\mathrm{s}_{\mathrm{L}}$ is set to 1 ; i.e., unless a full-scale model is used. Since Eq. (31) is needed for strain rate effects, a scale model made of the same material as the prototype will be unable to simulate the strain-rate effect.

The foregoing limitations on the use of identical materials for the scale model and the prototype have long been recognized in the literature of scale-model impact testing. However, since the use of different material to overcome these limitations is difficult and often impossible, almost all scale models use the same material as the prototype and ignore the effects of gravity and strain rate. When using the same material for the scale model, the possible effects of gravity and strain-rate on the measured results must be studied.

Although the use of different material for a scale model is not commonly practiced, the requirements for this procedure are also given in this report. The use of different material may be possible for package components whose material remains elastic during a drop test. If the gravitational acceleration cannot be changed and ignored, the use of different material will be the only alternative.

Table B-2 shows all the possible ways to satisfy the scaling laws given by Eqs.(1) through (27). Most of these possibilities rely on some approximations, i.e., neglecting one or more of the following effects: gravity, strain rate, mass, stiffness, wave and vibration. The use of these approximations will provide a greater flexibility in the design of the scale model. The results in Table B-2 are presented in terms of scale models for various types of impact response. The " $A$ " models are intended to measure complete impact response including waves and vibrations; the " $\mathrm{B}$ " and " $\mathrm{C}$ " models are for the rigid-mass and 
massless material responses, respectively. In each of these model families, several models are presented, using identical or different materials, ignoring gravity or strain-rate effects. For each of the scale models, Table B-2 gives the governing scaling laws and the method used to satisfy them; Table B-3 gives the scale factors for most of the parameters used in a drop test. The listed scale factors can be easily derived from the independent factors according to the dimension of the test parameters; the inclusion of many of the parameters is merely for convenience.

All the scale models described in the literature are included in Tables B-2 and B-3. Model A-4 satisfies the Scaling Law 2 of Soper (1964); the combination of Model B-2 and C-1 matches the situation described in the same paper for Scaling Laws 1 and 4, and the combination of Models B-2 and C-3 for Law 4 . Soper's scaling law 2 covers most of the scale models used for dynamic tests with large permanent deformations [Baker 1960, Ezra and Penning 1962, etc.] Almost all the scale models used for package drop tests are identical to Model A-4 [Goldman et al. 1989, Huerta \& Yoshimura 1976, Huerta 1978 and 1981, Robinson 1976, Yoshimura et al. 1989, Warrant 1987]. A few of the models use Model B-2 for the container and contents, and Model C-3 for the impact limiter [Nolan et al. 1989]. No attempt has been made to use different material for the scale model.

To apply the results of Tables B-2 and B-3 to the design of scale model, it must first be decided which impact response to simulate, then whether the gravity effect can be neglected and whether the material is rate-sensitive. If the wave and vibration responses are significant, one of the Models A must be chosen. If both the gravity and rate effect can be neglected, Model A-4, which uses the same material for the scale model as the prototype, is a good choice.

\section{B.4.3 Verification of Scale Models}

Each of the scale models listed in Tables B-2and $\mathrm{B}-3$ is designed to simulate one or more of the three impact responses: the wave, vibration and rigid-body responses. The SCANS computer program [Gehard et al. 1988] is used here to verify that the model indeed meets its intended purpose. The computer program is used to predict and compare the impact responses of the scale models and the prototype. Table B-4 lists the scale models studied and the impact responses they simulate. Each of the package scale models studied is a composite model which uses different scale models or scaling laws for different parts of the package, namely, the container, the contents, and the impact limiters. A composite model is used here to demonstrate that it is possible to use different scaling laws for different parts of the package as long as the scaling laws appropriately measure the dominant impact response for that part. This approach has been used by Soper 1964 to develop the Scaling Laws 1, 3, and 4 presented therein. It has also been used for scale-model testing of transport packages (Nolan et al. 1989).

By comparing the SCANS results for the container, the present study demonstrates the possible consequences of using Scale Models B-1, $A-3$, and $A-4$ to represent the container in a scalemodel test. As described in Table B-4, Model B-1 can provide reliable measurements of the rigidbody impact response only, whereas Models A-3 and $A-4$ should also be able to furnish reliable data for the wave and vibration responses. Model A-4 is less accurate than A-3, because the former does not simulate the gravity effect. However, A3 introduces the additional complication of using a different material for the scale model. The SCANS results confirm these expectations of the performance of the three models as presented in Table B-5 and Figures B1a-B4b for Casks 0003, 0103,0113 , and 0123. Cask 0003 is the prototype; 0103, 0113, and 0123 are the Scale Models B-1, $A-4$, and A-3, respectively. The results are for 30 $\mathrm{ft}$ end-on and 45-deg. oblique drops. Table B-5 shows that for the rigid-body response, both B-1 (Cask 0103) and A-4 (Cask 0123) give results very close to that of the prototype, whereas $A-4$ (Cask 0113) does not. Figures B-1-B-4, however, show that $B-1$ (Cask 0103) gives very different wave and vibration responses from the prototype. The wave and vibration responses are the superimposed oscillations in the presented force and stress-time histories. This poor performance of $\mathrm{B}-1$ for wave and vibration responses is even 
more obvious in the stress results with lead-slump effect.

In practice, Model A-3 (Cask 0124) gives better results for all impact responses than other models; however, the model may not be feasible because it requires different material for the scale model. If a similar material cannot be found and the wave and vibration response are more significant than the gravity effect, one might have to settle for Model A-4 (cask 0113). This is probably the reason why A-4 is used in most package-drop tests. This case represents one of the many practical situations where the designer of a scale model must use his understanding of the impact phenomenon and exercise his judgment of the relative importance of the existing effects in order to produce an optimal scale model.

\section{B.5 Other Considerations in Scale- Model Testing}

In practice, there are other constraints and considerations for scale model tests than the scaling laws. Some of these considerations are highlighted in this section.

\section{B.5.1 Environmental/initial conditions}

An implicit requirement for scale modeling is that the scale model is tested in an environment similar to the prototype's. This means that all environmental conditions such as ambient temperature, solar heat and humidity that are expected to have a significant effect on the property of the package materials and on the initial stress of the package must be properly scaled. The impact response of liquid contents will be drastically changed if the liquid is frozen at low temperature. A wet impact limiter made of wood will perform differently from a dry one. Since it is the mechanical behavior that controls the impact response of a package, the simulation of the environment should be based on the mechanical properties of the package materials. The environment for the scale model must be so adjusted that it produces similar initial mechanical behaviors between the scale model and the prototype.

\section{B.5.2 Target Characteristics}

Like the environmental/initial conditions, the target characteristics must also be considered an integral part of the test model. As discussed earlier in this report, the target behaves as a massive rigid body. In order to satisfy the requirement of "unyielding ground," the target used for a scale-model test must possess a sufficiently large mass, a sufficiently stiff impact area, and a geometry that does not cause significant wave and vibration response.

\section{B.5.3 Fabrication}

The method of fabrication can have a significant effect on the material property and this effect can vary with the size of the fabricated piece. Moreover, in some cases, the fabrication methods used for the prototype and the scale model must be different, because the fabrication method cannot be used for smaller products. For these reasons, it is necessary to identify and evaluate the significance of all possible effects of the fabrication method on the similarity of the models. Joseph (1983) has discussed some of the practical problems in the fabrication assembly and handling of scale models.

\section{B.5.4 Quality Assurance}

The acceptance criteria for the quality assurance program of the scale model should be properly converted from the prototype using the scaling laws. For example, the geometric tolerance and allowable defect size for the scale model must be obtained from the prototype using the scale factor for geometry. The sensitivity and accuracy of the instruments used for inspection should also be adjusted accordingly to assure equal quality between the prototype and the scale model.

\section{B.5.5 Instrumentation}

Like the instruments for quality inspection, the instruments for measuring the impact response of the scale model must also have the accuracy and sensitivity to meet the needs of the scale model, not the prototype. As shown in Table 4, the time scale in a reduced-scale model is often shorter than the prototype's. This fact means that the scale model will have shorter impact duration and higher natural vibration frequencies than the prototype. Thus, the frequency response range of the accelerometer and 
the instruments for time-history recording and processing must be correspondingly higher for the scale model.

The process of extracting the rigid-body response from the results of a drop test can be cited to demonstrate the need for adjusting the frequency response of data processing equipment. A common method for obtaining the rigid-body response of an impacting package is by filtering the actual timehistory records of a drop test [Yoshimura et al. 1989]. By using a low-pass or band-pass filter, the wave and vibration responses of a package can be eliminated to provide a fairly accurate time-history record of the rigid-body response. When this technique is used to process the results of a scale model, the natural vibration frequencies of the scale model-not the prototype-should be used to set the cut-off frequency of the filter.

\section{B.5.6 Statistical Significance of Measurements}

For many reasons, a scale model can never be uniformly similar to the prototype. Some of the reasons are the lack of complete knowledge of the underlying phenomena and controlling parameters, and the lack of ability to fabricate the model with the desired results. An example is a bolted joint. The response of a bolted joint depends on many factors, such as preload and surface roughness, that are difficult to measure and control. Therefore, one cannot expect a bolted joint to be reproduced with the same degree of certainty as the total mass of a package. This nonuniformity of the quality of the scale model would cause the test data to show various amounts of scatter. The impact response which depends on less-certain characteristics of the scale model will show greater scatter and less reliability. The measurements of system or global response (like the rigid-body acceleration of the package), would show less scatter than the local response (like the stress in the package), because the global response is affected by parameters of greater control (like the total mass). This difference between the global and local response data is generally supported by published data from drop tests.

Since a greater scatter in the scale-model test data indicates a greater uncertainty in the design of the scale model, and probably also in the prototype design, the scatter data can probably be used to estimate the available margin of safety of the design.

\section{B.5.7 Size Effects}

The microstructure of some materials like honeycomb can be comparable in size to the components of a scale model. In this case, the microstructure will also need scaling. However, this may be impossible because the behavior of a microstructure is usually complex and exhibits a size effect that invalidates scale-model testing.

\section{B.5.8 Test Confirmation of Scale Model and Scaling Laws}

Since the quality of a scale model can be unpredictable, it is essential to obtain direct experimental evidence to validate the scale model and scaling laws. The following are suggestions for obtaining the evidence:

- Measure the total weight and c.g. of the scale model to compare with the prototype

- Apply a load statically and measure the forcedisplacement relation of the scale model and compare with the prototype

- Measure the natural vibration frequencies of the scale model and compare with the prototype

- Test scale models of at least two different sizes and correlate the data using the scaling laws.

\section{B.6 Guidelines For Scale Model Testing}

Summing up the information presented in this report, the following guidelines are suggested for planning and conducting scale-model testing:

Before a scale model can be designed, the objective of the test to be conducted on the model must be clearly specified; i.e., whether it is a test to confirm an analysis or an analysis method, a test to prove the structural integrity of the package, or a test to demonstrate the compliance of the package with the regulatory requirements. The key measurements for the test must then be defined, and their competence in meeting the specified test objective be demonstrated. If it is a test to demonstrate integrity 
or compliance, the acceptance criteria, in terms of the specified measurements, should also be defined. The objective of the test also determines the complexity and quality control requirements for the scale model. In general, an integrity or a compliance test will require greater details and stricter controls.

To develop the required scaling laws for the specified measurements, the physical phenomena and parameters controlling these measurements must be identified. Depending on the design of the package, the impact response can be dominated by the rigid-body response or also by the wave and vibration responses. The modeling requirement for the rigid-body response is much less demanding. However, for all cases, the stiffness property of the package near the impact point plays an important role and must be well understood and characterized in order to develop the required scaling laws. In general, the condition of geometric similarity, kinematic similarity, dynamic similarity, gravitational similarity, and material similarity should be satisfied everywhere in the scale model. These conditions provide the necessary scaling laws for the desired measurements.

If a different material from the prototype is used for the scale model, the force-deformation behaviors of the material in the drop test must be well

understood. The dominant behavior of the prototype and scale-model materials must be similar and governed by a set of definitive constitutive laws characterized by measurable material constants. Scaling laws must be developed for all the influential material constants and used to select the scale-model material. As an example, the elastic behavior of most materials is governed by Hooke's law and characterized by two measurable constants: Young's modulus and Poisson's ratio. Scaling laws have been established here for these two constants and can be used to select a similar material for the scale model.

A scale model cannot be expected to simulate all the influencing phenomena; thus, the omission of some relatively insignificant effects is an acceptable approach. However, the omitted effects must be identified and the possible errors introduced by the omission must be estimated and taken into account in the usage of the results. The effects of gravity and strain rate must be addressed whether the same or different materials are used for the scale model and the prototype. It is highly desirable to use the test data and demonstrate the extent of the omitted effects directly.

All influential details of the scale model must be designed according to the scaling laws. The dimension tolerances of the scale model should be derived from the prototype using the scale factor for geometry. Scaling laws should also be observed to set the environment and test conditions, the target design, the sensitivity of instruments, and the data reduction procedure.

The fabrication methods for the scale model and the prototype must be described and compared. If the same method is used, it is necessary to demonstrate that the property of the finished piece does not vary with its size. If different methods are used, it is essential to show that the property does not vary significantly with the method.

A QA procedure and a set of acceptance standards must be prepared specifically for the scale model. The standards must guarantee similar quality between the scale model and the prototype.

A test procedure must be prepared for the scale model test, specifying the environment and test conditions, the instruments, and the data collection and reduction procedures.

The number of specimens and tests must be sufficient to show the scatter or the statistical nature of the measured data. The requirement is especially important for data that are affected by localized properties which cannot be easily modeled and controlled. The scatter of the data indicates the overall effectiveness and reliability of the scale model test, including the design and fabrication of the scale model. For some cases, the scatter would also be useful for determining the necessary margin of safety for the package design.

Tests should also be carried out using models of different size or scale to provide a direct verification of the scaling laws used for the test. 
Table B-1 Common Package Design and Expected Dominant Impact Response

EXPECTED DOMINANT

PACKAGE DESIGN IMPACT RESPONSE

Container/contents without impact limiter

All including vibration/wave response

Container/contents with limiter of comparable mass \& stiffness

All including vibration/wave response in container/contents and limiter

Stiff container \& contents with soft impact limiter

Container/contents: rigid body response

Limiter: all including vibration/wave response

Stiff container \& contents with light-weight $\quad$ Containcr/contents: rigid body response soft impact limiter

Limiter: massless material reponse 
Table B-2 Scale Models for Drop Tests

\begin{tabular}{|c|c|c|c|}
\hline $\begin{array}{l}\text { SCALE } \\
\text { MODEL }\end{array}$ & $\begin{array}{c}\text { EFFECTS } \\
\text { OMMITIED IN } \\
\text { MODEL }\end{array}$ & $\begin{array}{l}\text { GOVERNING } \\
\text { SCALING LAWS }\end{array}$ & $\begin{array}{l}\text { APPROACH TO } \\
\text { SATISFYING } \\
\text { SCALING LAW }\end{array}$ \\
\hline
\end{tabular}

\section{SCALE MODELS FOR COMPLETE IMPACT RESPONSE, INCLUDING} WAVE AND VIBRATION

A-1 None

A-2

\section{Strain-rate}

dependence of

material stiffness
- Geometric similarity

- Kinematic similarity

- Dynamic similarity

- Gravitational similarity

- Material similarity in both mass and stiffness properties

- Geometric similarity

- Kinematic similarity

- Dynamic similarity

- Gravitational similarity

- Material similarity in both mass and rate-independent stiffness properties

A-3 Strain-rate
dependence of
material stiffness

Both strain-rate and gravity effects

- Kinematic similarity
- Geometric similarity

- Dynamic similarity

- Gravitational similarity

- Material similarity in both mass and ralc-independent stiffness properties

- Geometric similarity

- Kinematic similarity

- Dynamic similarity

- Material similarity in both mass and rate-independent stiffness properties
- Change material and gravitational acceleration

- Use same material but change gravitational acceleration

- Use different material 
Table B-2 Scale Models for Drop Tests, cont'd.

\begin{tabular}{|c|c|c|c|}
\hline $\begin{array}{l}\text { SCALE } \\
\text { MODEL }\end{array}$ & $\begin{array}{l}\text { EFFECTS } \\
\text { OMMITTED IN } \\
\text { MODEL } \\
\end{array}$ & $\begin{array}{c}\text { GOVERNING } \\
\text { SCALING LAWS } \\
\end{array}$ & $\begin{array}{l}\text { APPROACH TO } \\
\text { SATISFYING } \\
\text { SCALING LAW } \\
\end{array}$ \\
\hline \multicolumn{4}{|c|}{ SCALE MODELS FOR RIGID-BODY IMPACT RESPONSE } \\
\hline B-1 & None & $\begin{array}{l}\text { - Geometric similarity } \\
\text { - Kinematic similarity } \\
\text { - Dynamic similarity } \\
\text { - Gravitational similarity } \\
\text { - Material similarity in mass } \\
\text { property only }\end{array}$ & - Change gravitational acceleration \\
\hline B-2 & Gravity cffects & $\begin{array}{l}\text { - Gcometric similarity } \\
\text { - Kinematic similarity } \\
\text { - Dynamic similarity } \\
\text { - Material similarity in mass } \\
\text { property only }\end{array}$ & $\begin{array}{l}\text { - Scale factors for length, time, and } \\
\text { mass can be independently } \\
\text { selected }\end{array}$ \\
\hline \multicolumn{4}{|c|}{ SCALE MODELS FOR MASSLESS-MATERIAL RESPONSE } \\
\hline $\mathrm{C}-1$ & None & $\begin{array}{l}\text { - Geometric similarity } \\
\text { - Kinematic similarity } \\
\text { - Material similarity in stiffness } \\
\text { property only }\end{array}$ & $\begin{array}{l}\text { - Use same material and keep time } \\
\text { scale unchanged }\end{array}$ \\
\hline $\mathrm{C}-2$ & None & $\begin{array}{l}\text { - Gcomctric similarity } \\
\text { - Kinematic similarity } \\
\text { - Material similarity in stiffness } \\
\text { property only }\end{array}$ & - Use different material \\
\hline C-3 & Strain-ratc effects & $\begin{array}{ll}- & \text { Gcometric similarity } \\
- & \text { Kinematic similarity } \\
\text { - Material similarity in rate- } \\
\text { independent property only }\end{array}$ & - Use same material \\
\hline C-4 & Strain-rate effects & $\begin{array}{l}\text { - Geometric similarity } \\
\text { - Kinematic similarity } \\
\text { - Material similarity in rate- } \\
\text { independent property only }\end{array}$ & - Use different material \\
\hline
\end{tabular}


Table B-3 Scaling Factors for Scale-Model Impact Test

SCALE FACTOR FOR PARAMETER

\begin{tabular}{|c|c|c|c|c|c|c|c|c|c|c|c|}
\hline \multirow[b]{2}{*}{ PARAMETER } & \multirow[b]{2}{*}{$\begin{array}{l}\text { PARAMETER } \\
\text { DIMENSION }\end{array}$} & \multicolumn{9}{|c|}{ SCALE FACTOR FOR PARAMETER } & \multirow[b]{2}{*}{$\begin{array}{c}\text { MODE } \\
\text { C-4 }\end{array}$} \\
\hline & & $\begin{array}{c}\text { MODEL } \\
\text { A-1 }\end{array}$ & $\begin{array}{c}\text { MODEL } \\
\text { A-2 }\end{array}$ & $\begin{array}{c}\text { MODEL } \\
\text { A-3 }\end{array}$ & $\begin{array}{c}\text { MODEL } \\
\text { A-4 }\end{array}$ & $\begin{array}{c}\text { MODEL } \\
\text { B }-1\end{array}$ & $\begin{array}{c}\text { MODEL } \\
\text { B-2 }\end{array}$ & $\begin{array}{c}\text { MODEL } \\
\mathrm{C}-1\end{array}$ & $\begin{array}{l}\text { MODEL } \\
\text { C-2 }\end{array}$ & $\begin{array}{c}\text { MODEL } \\
\text { C-3 }\end{array}$ & \\
\hline \multicolumn{2}{|c|}{ BASIC DIMENSIONS } & $s_{L}$ & & & & & & & & & \\
\hline Length & L & $s_{L}$ & $s_{L}=s_{g}^{-1}$ & $s_{L}$ & $s_{L}$ & $s_{L}$ & $s_{L}$ & $s_{L}$ & $s_{L}$ & $s_{L}$ & $s_{L}$ \\
\hline Time & $\mathrm{t}$ & $\left(s_{1} / s_{g}\right)^{.5}$ & $s_{\mathrm{L}}$ & $s_{L}^{5}$ & $s_{L}$ & $\left(s_{L} / s_{g}\right)^{.5}$ & $s_{t}$ & 1 & $s_{t}$ & $s_{t}$ & $s_{l}$ \\
\hline Mass & $\left(\mathrm{Ft}^{2}\right) / \mathrm{L}$ & $s_{F} / s_{g}$ & $s_{L}^{3}$ & $s_{\mathrm{F}}$ & $s_{L}^{3}$ & $s_{F} / s_{g}$ & $\left(s_{f} s_{t}^{2}\right) / s_{L}$ & - & - & - & - \\
\hline Force & $\mathrm{F}$ & $s_{\mathrm{F}}$ & $s_{\mathrm{L}}^{2}$ & $s_{F}$ & $s_{L}^{2}$ & $\mathrm{~s}_{\mathrm{F}}$ & $\mathrm{s}_{\mathrm{F}}$ & $\mathrm{s}_{\mathrm{L}}^{2}$ & $\mathrm{~s}_{\mathrm{F}}$ & $s_{L}^{2}$ & $\mathrm{~s}_{\mathrm{F}}$ \\
\hline
\end{tabular}

Table B-3 Scaling Factors for Scale-Model Impact Test, cont'd.

SCALE FACTOR FOR PARAMETER

\begin{tabular}{|c|c|c|c|c|c|c|c|c|c|c|c|}
\hline PARAMETER & $\begin{array}{c}\text { PARAMETER } \\
\text { DIMENSION } \\
\end{array}$ & $\begin{array}{c}\text { MODEL } \\
\mathrm{A}-1\end{array}$ & $\begin{array}{c}\text { MODEL } \\
\mathrm{A}-2 \\
\end{array}$ & $\begin{array}{c}\text { MODEL } \\
\text { A-3 }\end{array}$ & $\begin{array}{c}\text { MODEL } \\
\mathrm{A}-4 \\
\end{array}$ & $\begin{array}{c}\text { MODEL } \\
\mathrm{B}-1\end{array}$ & $\begin{array}{c}\text { MODEL } \\
\text { B-2 }\end{array}$ & $\begin{array}{c}\text { MODEL } \\
\mathrm{C}-1\end{array}$ & $\begin{array}{c}\text { MODEL } \\
\text { C-2 } \\
\end{array}$ & $\begin{array}{c}\text { MODEL } \\
\mathrm{C}-3\end{array}$ & $\begin{array}{c}\text { MODEL } \\
\text { C-4 }\end{array}$ \\
\hline \multicolumn{12}{|c|}{ TEST CONDITIONS } \\
\hline Drop height & L & $s_{L}$ & $s_{L}$ & $s_{L}$ & 1 & $s_{L}$ & $s_{L}$ & - & - & - & - \\
\hline Impact velocity & $\mathrm{L} / \mathrm{t}$ & $\left(s_{L} s_{g}\right)^{.5}$ & 1 & $s_{L}^{5}$ & 1 & $\left(s_{L} s_{g}\right)^{.5}$ & $s_{L} / s_{1}$ & - & - & - & - \\
\hline Impact angle & - & 1 & 1 & 1 & 1 & 1 & $\because 1$ & & & & \\
\hline Accel. of gravity & $\mathrm{L} / \mathrm{t}^{2}$ & $s_{\mathrm{g}}$ & $s_{g}$ & $s_{g}$ & - & $\mathrm{s}_{\mathrm{g}}$ & - & - & - & - & - \\
\hline Applied force & F & $s_{F}$ & $s_{\mathrm{L}}^{2}$ & $\mathrm{~s}_{\mathrm{F}}$ & $s_{L}^{2}$ & $s_{F}$ & $s_{F}$ & $s_{L}^{2}$ & $\mathrm{~s}_{\mathrm{F}}$ & $s_{\mathrm{L}}^{2}$ & $s_{F}$ \\
\hline
\end{tabular}


Table B-3 Scaling Factors for Scale-Model Impact Test, cont'd.

SCALE FACTOR FOR PARAMETER

\begin{tabular}{|c|c|c|c|c|c|c|c|c|c|c|c|}
\hline \multirow[b]{2}{*}{ PARAMETER } & \multirow[b]{2}{*}{$\begin{array}{l}\text { PARAMETER } \\
\text { DIMENSION } \\
\end{array}$} & & & & & & & & & & \\
\hline & & $\begin{array}{c}\text { MODEL } \\
\text { A-1 }\end{array}$ & $\begin{array}{c}\text { MODEL } \\
\mathrm{A}-2\end{array}$ & $\begin{array}{c}\text { MODEL } \\
\text { A-3 }\end{array}$ & $\begin{array}{c}\text { MODEL } \\
\text { A-4 }\end{array}$ & $\begin{array}{c}\text { MODEL } \\
\text { B-1 }\end{array}$ & $\begin{array}{c}\text { MODEL } \\
\text { B-2 }\end{array}$ & $\begin{array}{c}\text { MODEL } \\
\text { C-1 }\end{array}$ & $\begin{array}{c}\text { MODEL } \\
\text { C-2 }\end{array}$ & $\begin{array}{c}\text { MODELL } \\
\text { C-3 }\end{array}$ & $\begin{array}{c}\text { MODEL } \\
\text { C-4 }\end{array}$ \\
\hline \multicolumn{12}{|c|}{ MODEL GEOMETRY } \\
\hline C.G. coord. & L & $\mathrm{s}_{\mathrm{L}}$ & $\mathrm{SL}_{\mathrm{L}}$ & $\mathrm{SL}_{\mathrm{L}}$ & $\mathrm{S}_{\mathrm{L}}$ & $\mathrm{s}_{\mathrm{L}}$ & $\mathrm{S}_{\mathrm{L}}$ & $\mathrm{s}_{\mathrm{L}}$ & $\mathrm{s}_{\mathrm{L}}$ & $\mathrm{s}_{\mathrm{L}}$ & $\mathrm{s}_{\mathrm{L}}$ \\
\hline $\begin{array}{l}\text { Impact pt. } \\
\text { coord. }\end{array}$ & L & $\mathrm{S}_{\mathrm{L}}$ & $\mathrm{s}_{\mathrm{L}}$ & $\mathrm{s}_{\mathrm{L}}$ & $\mathrm{S}_{\mathrm{L}}$ & $\mathrm{s}_{\mathrm{L}}$ & $\mathrm{S}_{\mathrm{L}}$ & $\mathrm{s}_{\mathrm{L}}$ & $\mathrm{s}_{\mathrm{L}}$ & $s_{L}$ & $\mathrm{~s}_{\mathrm{L}}$ \\
\hline $\begin{array}{l}\text { Load appl. pt. } \\
\text { coord. }\end{array}$ & L & $\mathrm{s}_{\mathrm{L}}$ & $s_{L}$ & $s_{L}$ & $s_{L}$ & $\mathrm{~s}_{\mathrm{L}}$ & $\mathrm{s}_{\mathrm{L}}$ & $\mathrm{s}_{\mathrm{L}}$ & $s_{L}$ & $s_{L}$ & $\mathrm{~s}_{\mathrm{L}}$ \\
\hline $\begin{array}{l}\text { Measurement } \\
\text { pt. coord. }\end{array}$ & L & $\mathrm{S}_{\mathrm{L}}$ & $\mathrm{s}_{\mathrm{L}}$ & $\mathrm{SL}_{\mathrm{L}}$ & $\mathrm{SL}_{\mathrm{L}}$ & $\mathrm{s}_{\mathrm{L}}$ & $\mathrm{s}_{\mathrm{L}}$ & $\mathrm{s}_{\mathrm{L}}$ & $\mathrm{s}_{\mathrm{L}}$ & $\mathrm{s}_{\mathrm{L}}$ & $s_{L}$ \\
\hline Area & $\mathrm{L}^{2}$ & $s_{L}^{2}$ & $s_{L}^{2}$ & $s_{l}^{2}$ & $s_{L}^{2}$ & $s_{L}^{2}$ & $s_{L}^{2}$ & $s_{\mathfrak{L}}^{2}$ & $s_{L}^{2}$ & $s_{L}^{2}$ & $s_{L}^{2}$ \\
\hline Volume & $\mathrm{L}^{3}$ & $s_{L}^{3}$ & $s_{L}^{3}$ & $s_{L}^{3}$ & $s_{L}^{3}$ & $s_{L}^{3}$ & $s_{L}^{3}$ & $s_{L}^{3}$ & $s_{L}^{3}$ & $s_{L}^{3}$ & $s_{L}^{3}$ \\
\hline
\end{tabular}


Table B-3 Scaling Factors for Scale-Model Impact Test, cont'd.

SCALE FACTOR FOR PARAMETER

\begin{tabular}{|c|c|c|c|c|c|c|c|c|c|c|c|}
\hline \multirow[b]{2}{*}{ PARAMETER } & \multirow[b]{2}{*}{$\begin{array}{l}\text { PARAMETER } \\
\text { DIMENSION }\end{array}$} & \multicolumn{9}{|c|}{ SCALE FACTOR FOR PARAMETER } & \multirow[b]{2}{*}{$\begin{array}{c}\text { MODEL } \\
\mathrm{C}-4\end{array}$} \\
\hline & & $\begin{array}{c}\text { MODEL } \\
\mathrm{A}-1\end{array}$ & $\begin{array}{c}\text { MODEL } \\
\mathrm{A}-2 \\
\end{array}$ & $\begin{array}{c}\text { MODEL } \\
\text { A-3 }\end{array}$ & $\begin{array}{c}\text { MODEL } \\
\text { A-4 }\end{array}$ & $\begin{array}{c}\text { MODEL } \\
\text { B-1 }\end{array}$ & $\begin{array}{c}\text { MODEL } \\
\text { B-2 }\end{array}$ & $\begin{array}{c}\text { MODEL } \\
\text { C-1 }\end{array}$ & $\begin{array}{c}\text { MODEL } \\
\text { C-2 } \\
\end{array}$ & $\begin{array}{c}\text { MODEL } \\
\text { C-3 }\end{array}$ & \\
\hline \multicolumn{12}{|c|}{ MATERIAL PROPERTY -MASS } \\
\hline $\begin{array}{l}\text { Total trans. } \\
\text { mass }\end{array}$ & $\left(\mathrm{Ft}^{2}\right) / \mathrm{L}$ & $s_{F} / s_{g}$ & $s_{L}^{3}$ & $s_{F}$ & $s_{L}^{3}$ & $s_{F} / s_{g}$ & $\left(s_{F} s_{t}^{2}\right) / s_{L}$ & - & - & - & - \\
\hline Total rot. mass & $\mathrm{Ft}^{2} \mathrm{~L}$ & $\left(s_{F} s_{L}^{2}\right) / s_{g}$ & $s_{L}^{5}$ & $\mathrm{~s}_{\mathrm{F}} \mathrm{s}_{\mathrm{L}}^{2}$ & $s_{L}^{5}$ & & $s_{F} s_{t}^{2} s_{L}$ & - & - & - & - \\
\hline $\begin{array}{l}\text { Mass density, } \\
P\end{array}$ & $\left(\mathrm{Ft}^{2}\right) / \mathrm{L}^{4}$ & $s_{F} /\left(s_{g} s_{L}^{3}\right)$ & 1 & $\mathrm{~s}_{\mathrm{F}} \mathrm{s}_{\mathrm{L}}^{3}$ & 1 & - & - & - & - & - & - \\
\hline \multicolumn{12}{|c|}{ MATERIAL PROPERTY $\rightarrow$ STIFFNESS } \\
\hline $\begin{array}{l}\text { "Modulus" } \\
\text { constants, E }\end{array}$ & $F / L^{2}$ & $s_{\mathrm{F}} / s_{\mathrm{L}}^{2}$ & 1 & $s_{F} / s_{L}^{2}$ & 1 & - & - & 1 & $s_{F} / s_{L}^{2}$ & 1 & $s_{F} / s_{L}^{2}$ \\
\hline $\begin{array}{l}\text { "Ratio" } \\
\text { constints, N }\end{array}$ & - & 1 & 1 & 1 & 1 & - & - & 1 & 1 & 1 & 1 \\
\hline $\begin{array}{l}\text { "Stress" } \\
\text { constints. K }\end{array}$ & $F / L^{2}$ & $\mathrm{~s}_{\mathrm{F}} / \mathrm{s}_{\mathrm{L}}^{2}$ & 1 & $s_{F} / s_{L}^{2}$ & 1 & - & - & 1 & $s_{F} / s_{L}^{2}$ & 1 & $s_{F} / s_{L}^{2}$ \\
\hline $\begin{array}{l}\text { "Viscosity" } \\
\text { constants, H }\end{array}$ & $F^{\prime}\left(L^{2} t\right)$ & $\left(s_{F} s_{g}^{s .5}\right) / s_{L}^{2.5}$ & & & & - & - & 1 & $s_{F} /\left(s_{g}^{2} s_{L}\right)$ & - & - \\
\hline
\end{tabular}




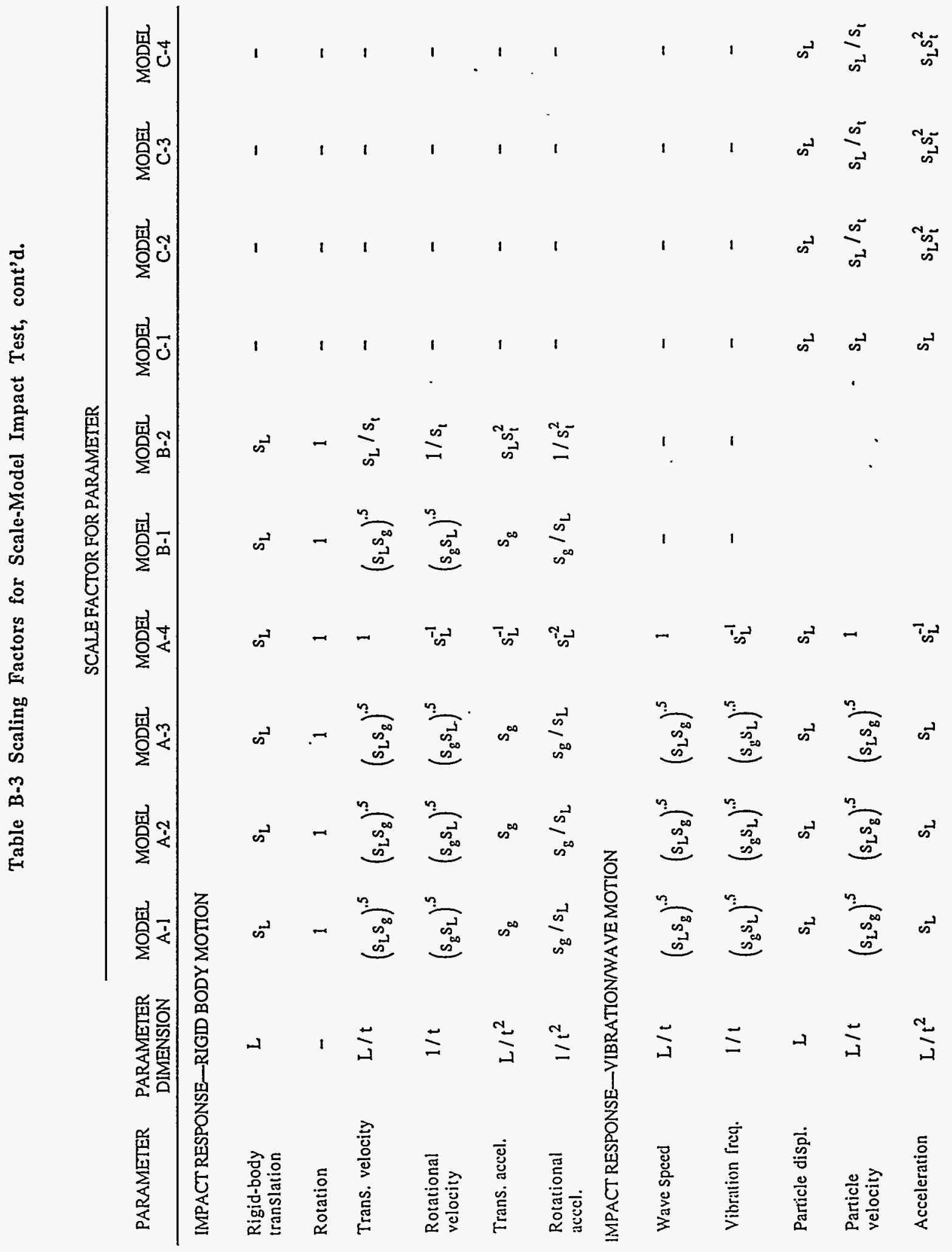


Table B-3 Scaling Factors for Scale-Model Impact Test, cont'd.

SCALE FACTOR FOR PARAMETER

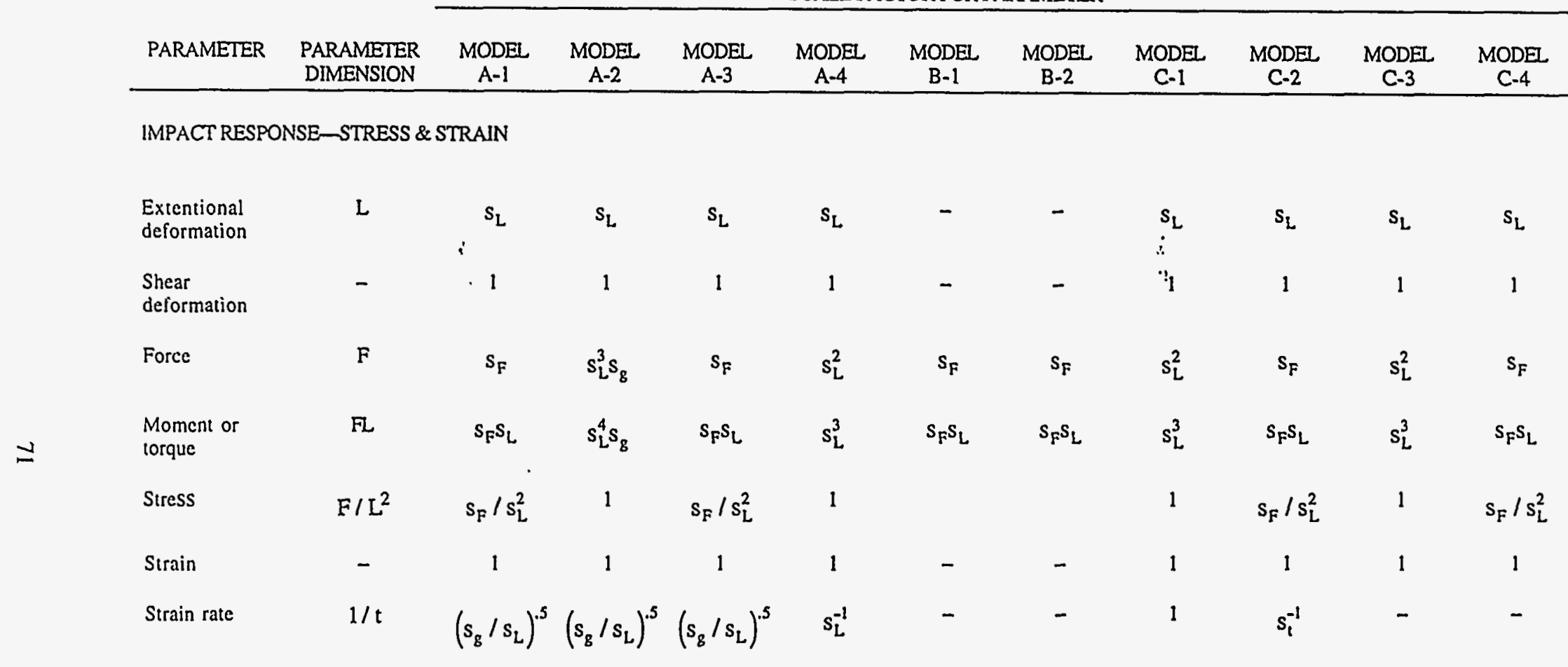


Appendix B: Scale-Model Impact Testing

Table B-4 Scale Models Used in the SCANS Study

SCALE MODEL FOR
CASK ID
$s_{L}$ CONTAINER CONTENTS LIMITER
EXPECTED MODEL
PERFORMANCE

\begin{tabular}{lll}
\hline $0003 \quad$ Prototype 11 & $\begin{array}{l}\text { The elastic response of the } \\
\text { container is to be studied using } \\
\text { the scale models. The limiter } \\
\text { deforms plastically. }\end{array}$
\end{tabular}

0103

Scale Model $\quad 4 / 30$

\section{Model B-1 \\ with same \\ material
. Model B-1 with same material

$s_{g}=1$

$\mathrm{S}_{\mathrm{F}}=\mathrm{s}_{\mathrm{L}}^{3}$

$s_{g}=1$

sp $=s^{2}$
Model C-4 with diff. material

$\mathrm{s}_{\mathrm{t}}=\mathrm{s}_{\mathrm{L}}^{5}$

$\mathrm{SF}_{\mathrm{F}}=\mathrm{s}_{\mathrm{L}}^{3}$

The model will provide good results for the rigid-body motion but not the vibration/wave response.

The accuracy of the results will not vary with the impact velocity, because there is no strain-rate effect and the gravity effect is properly modeled.

Model A-4 with same material

Model B-2 with same material $s_{t}=s_{L}$ $\mathrm{s}_{\mathrm{F}}=\mathrm{s}_{\mathrm{L}}^{2}$
Model C-3 with same material $s_{t}=s_{L}$ $s_{1}$

2

Model A-3

Model B-1

Model C-3 with with diff. with diff. same material material material $\mathrm{s}_{\mathrm{g}}=1$ $s_{g}=1$ $s_{t}=s_{L}^{.5}$ $\mathrm{s}_{\mathrm{F}}=\mathrm{s}_{\mathrm{L}}^{2}$ $\mathrm{s}_{\mathrm{F}}=\mathrm{s}_{\mathrm{L}}^{2}$

The model will provide good

The model will provide adequate results for the rigidbody motion and the vibration/wave response.

The accuracy of the results, however, will vary with the impact velocity, because the gravity effect is not properly modeled. results for the rigid-body motion and the vibration/wave response.

The accuracy of the results will not vary with the impact velocity, because there is no strain-rate effect and the gravity effect is properly modeled. 
Table B-5 Comparison of Rigid-Body Motions of Prototype and Scale-Model of Rail Cask

PROTOTYPE

RIGID-BODY

RESPONSE

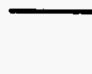

$\begin{array}{llll}\text { CASK } 0003 & \text { CASK } 0103 & \text { CASK } 0113 & \text { CASK } 0123\end{array}$

30-FT END-ON DROP-PRIMARY IMPACT

\begin{tabular}{|c|c|c|c|c|}
\hline Max. tran. accel. (g) & 45.5 & 45.6 & 40.0 & 45.5 \\
\hline $\begin{array}{l}\text { Max. rot. accel. } \\
\left(\mathrm{rad} / \mathrm{sec}^{* *} 2\right)\end{array}$ & 0.0 & 0.0 & 0.0 & 0.0 \\
\hline Max. Limiter crush (in) & 26.5 & 26.3 & 26.3 & 26.3 \\
\hline Max. impact force (kip) & 9150.0 & 9155.0 & 7898.0 & 9152.0 \\
\hline $\begin{array}{l}\text { Max. impact moment } \\
\text { (in-kip) }\end{array}$ & 0 & 0 & 0 & 0 \\
\hline \multicolumn{5}{|c|}{ 30-FT 45-DEG. OBLIQUE DROP-PRIMARY IMPACT } \\
\hline Max. tran. accel. (g) & 18.7 & 18.8 & 17.8 & .18 .7 \\
\hline $\begin{array}{l}\text { Max. rot. accel. } \\
\left(\mathrm{rad} / \mathrm{sec}^{* * 2}\right)\end{array}$ & 94.2 & 94.3 & 85 & 94.4 \\
\hline Max. Limiter crush (in) & 21.9 & 21.8 & 21.0 & 21.8 \\
\hline \multicolumn{5}{|l|}{ Max. impact force (kip) } \\
\hline Axial & 2428.0 & 2447.0 & 2211.0 & 2424.0 \\
\hline Shear & 3037.0 & 3038.0 & 2751.0 & 3032.0 \\
\hline $\begin{array}{l}\text { Max. impact moment } \\
\text { (in-kip) }\end{array}$ & 71973.0 & 72141.0 & 65559.0 & 71845.0 \\
\hline \multicolumn{5}{|c|}{ 30-FT 45-DEG. OBLIQUE DROP-SECONDARY IMPACT } \\
\hline Max. tran. accel. (g) & 12.1 & 12.1 & 11.9 & 12.1 \\
\hline $\begin{array}{l}\text { Max. rot. accel. } \\
\left(\mathrm{rad} / \sec ^{* * 2}\right)\end{array}$ & 111.3 & 111.4 & 101.3 & 111.6 \\
\hline Max. Limiter crush (in) & 16.0 & 15.8 & 12.8 & 15.8 \\
\hline \multicolumn{5}{|l|}{ Max. impact force (kip) } \\
\hline Axial & 253.0 & 253.0 & 298.0 & 253.0 \\
\hline Shear & 2557.0 & 2573.0 & 2357.0 & 2559.0 \\
\hline $\begin{array}{l}\text { Max. impact moment } \\
\text { (in-kip) }\end{array}$ & 11624.0 & 11707.0 & 9577.0 & 11644.0 \\
\hline
\end{tabular}



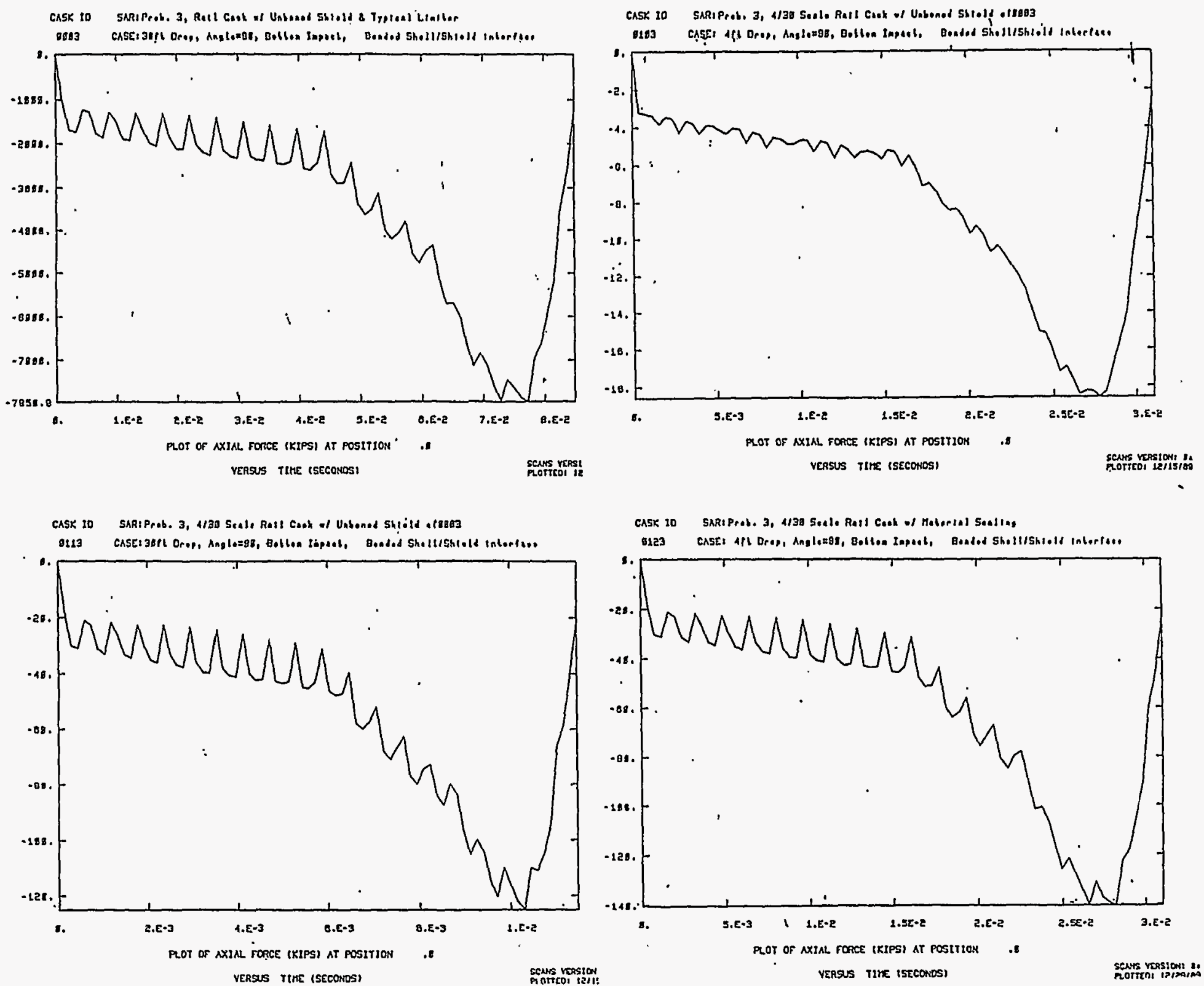

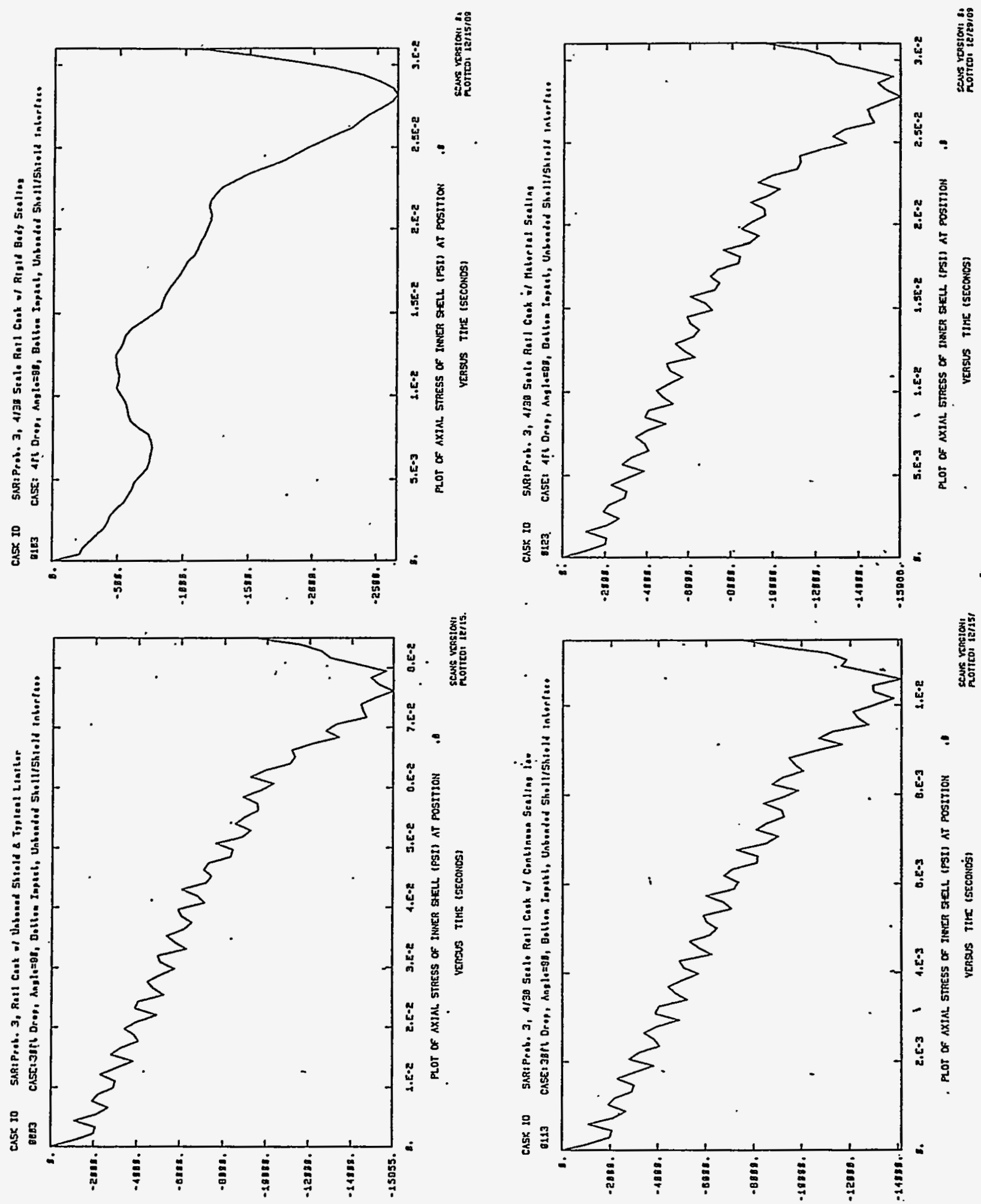

Figure B-2a Comparison of Impact Response of Prototype and Scale Models of Rail Cask with Lead Slump - 30-ft. End-On Drop, Time History of Axial Impact Stress in Inner Container Shell 


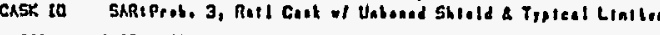

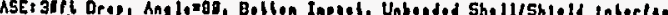

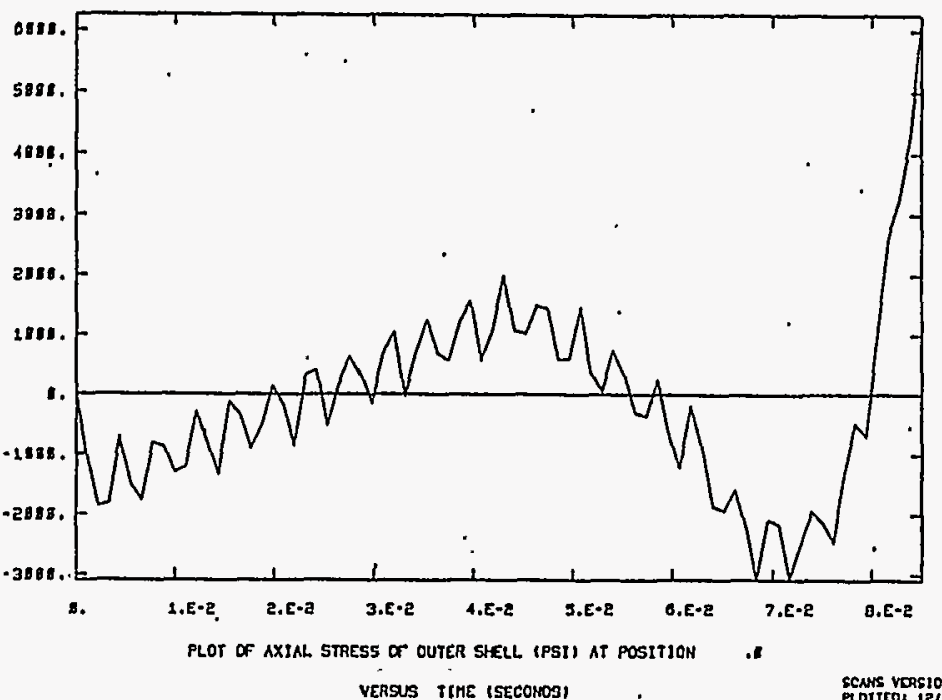

veRSUS TtME (sECONOS)

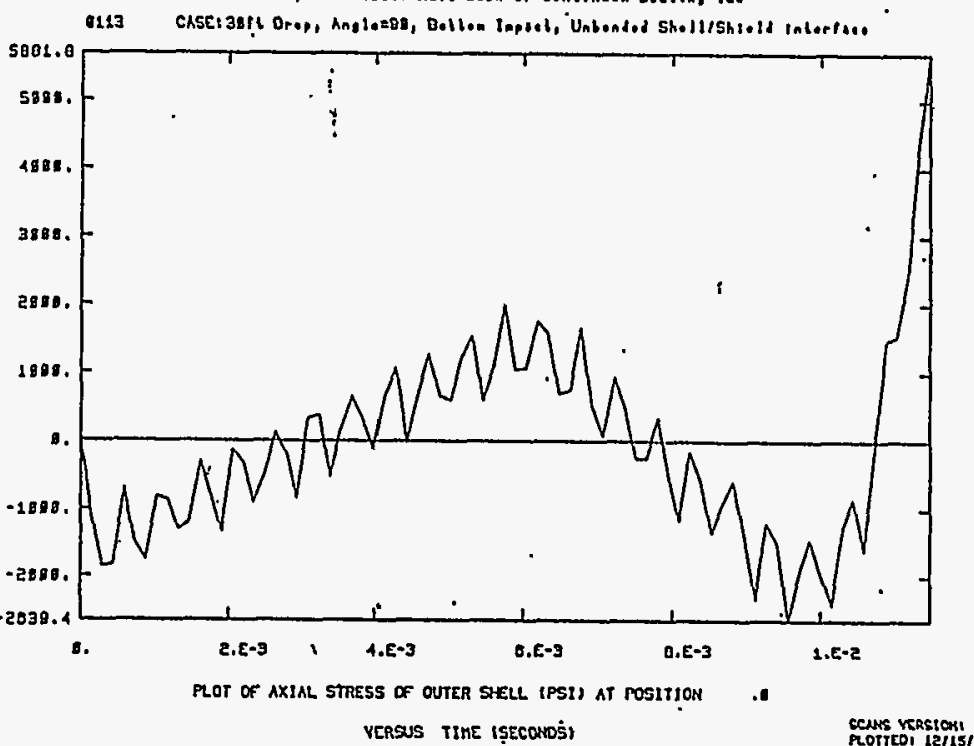

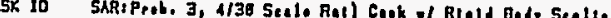

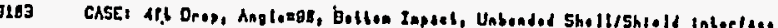

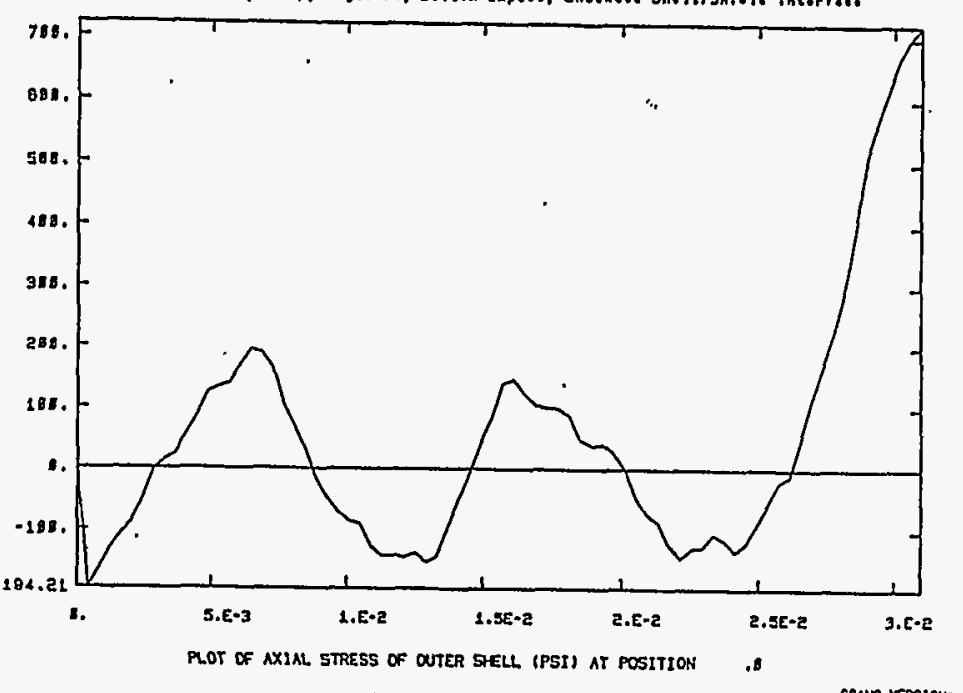

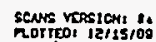

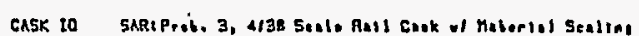

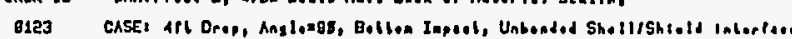

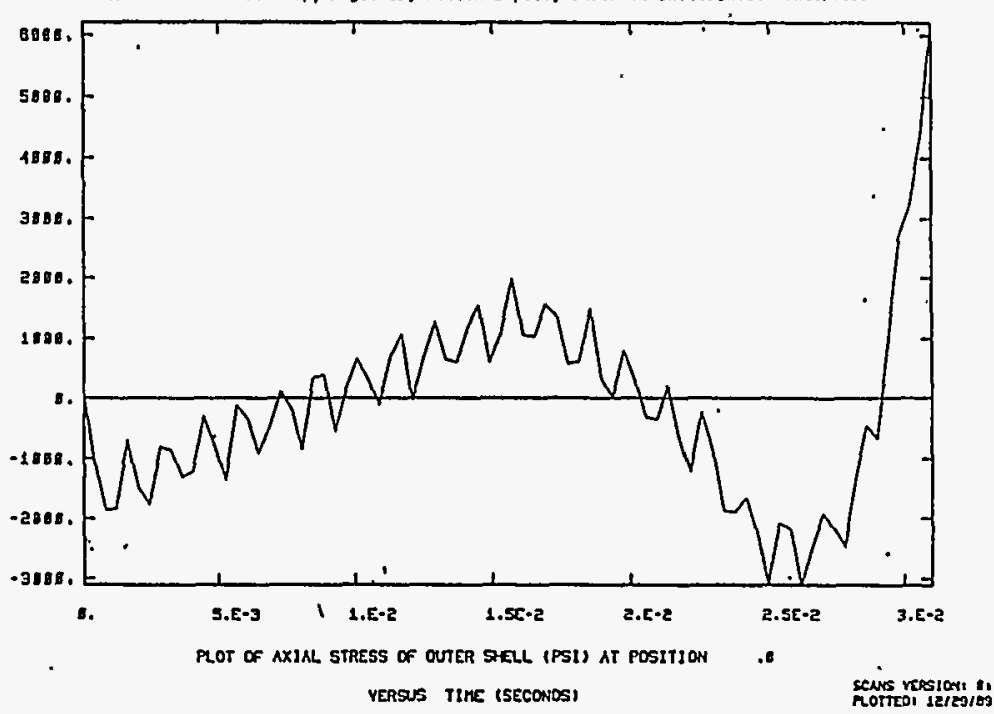



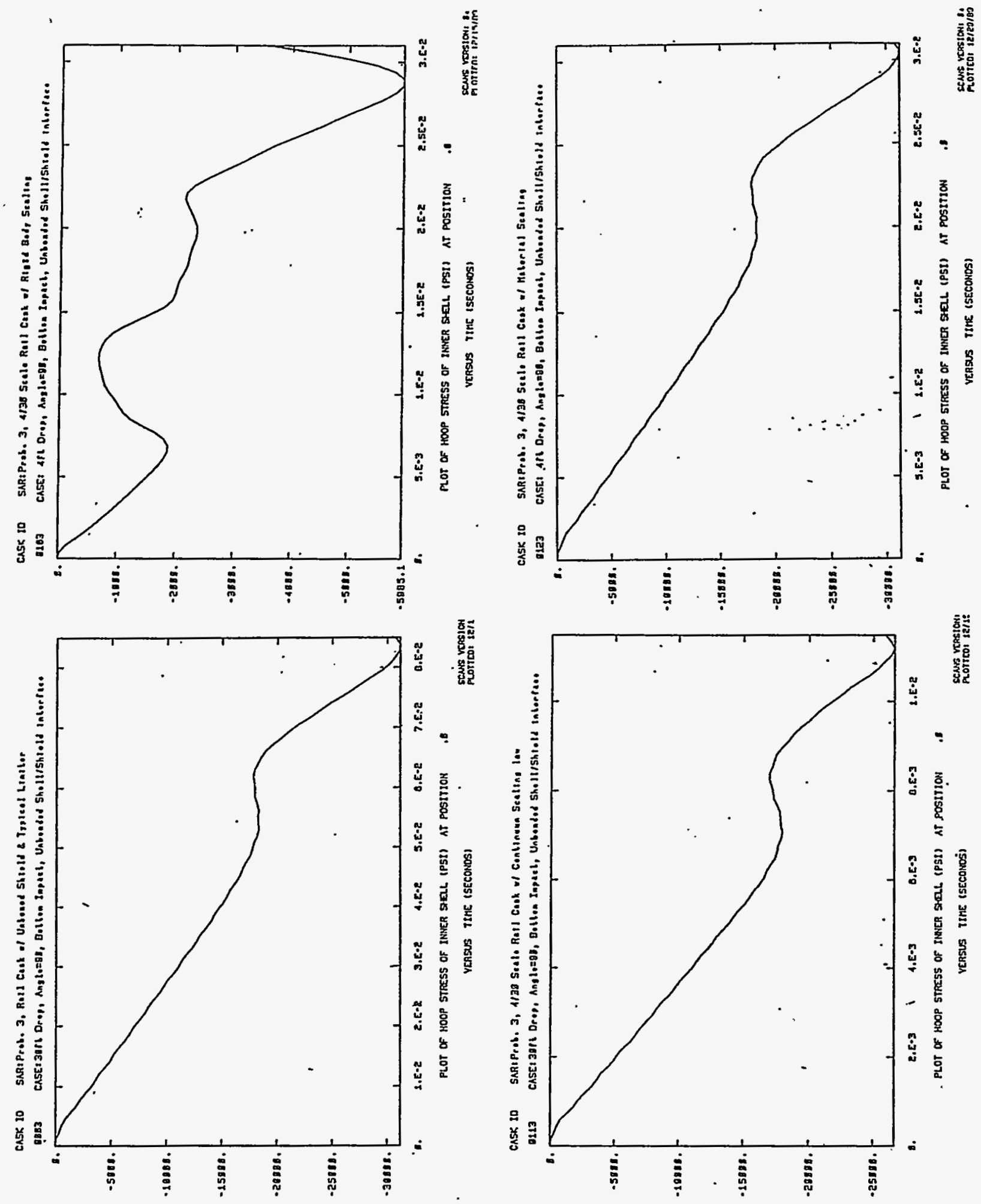

Figure B-3a Comparison of Impact Response of Prototype and Scale Models of Rail Cask with Lead Slump-30-ft. End-On Drop, Time History of Hoop Impact Stress in Inner Container Shell 

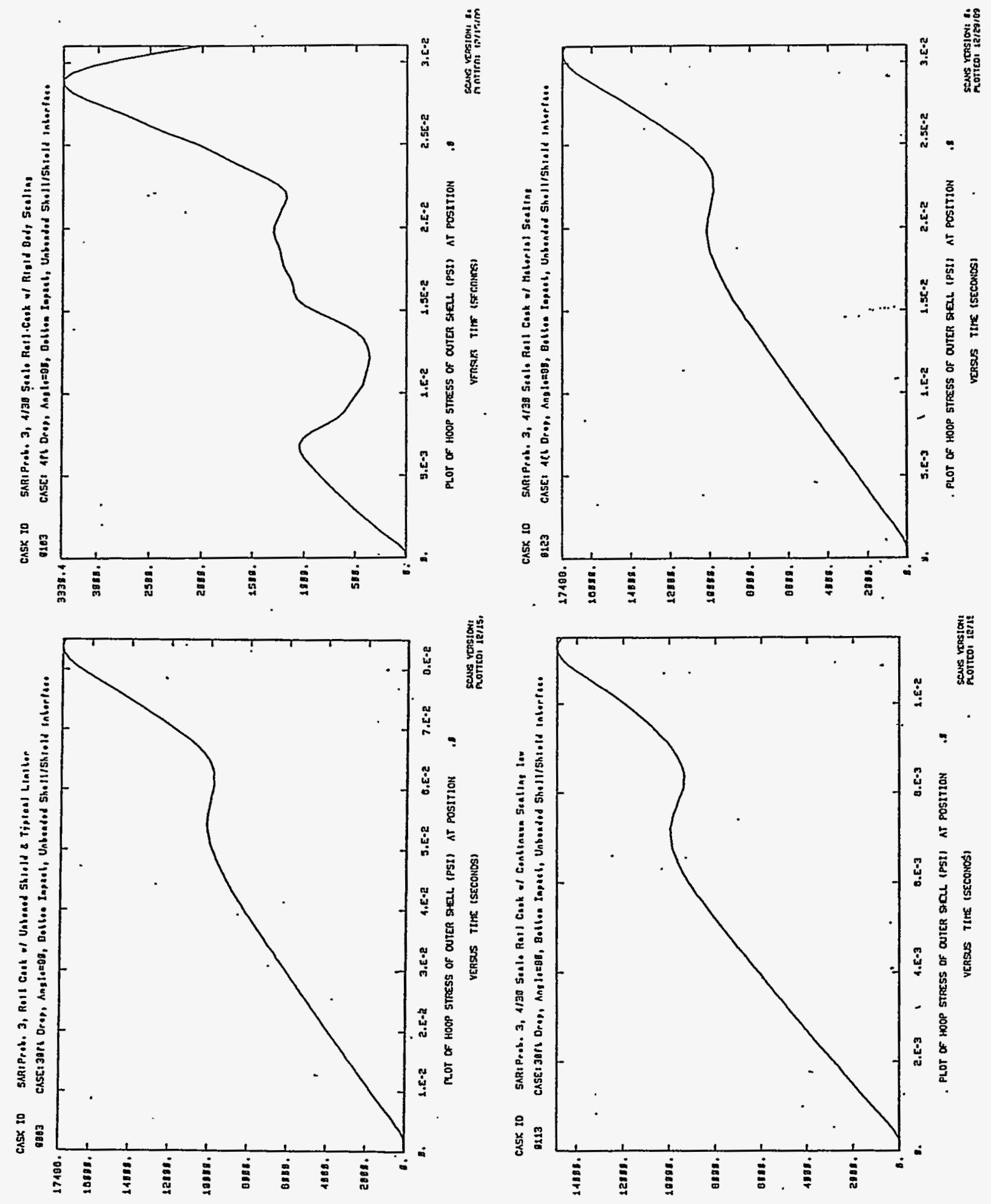

Figure B-3b Comparison of Impact Response of Prototype and Scale Models of Rail Cask with Lead Slump-30-ft. End-On Drop, Time History of Hoop Impact Stress in Outer Container Shell 

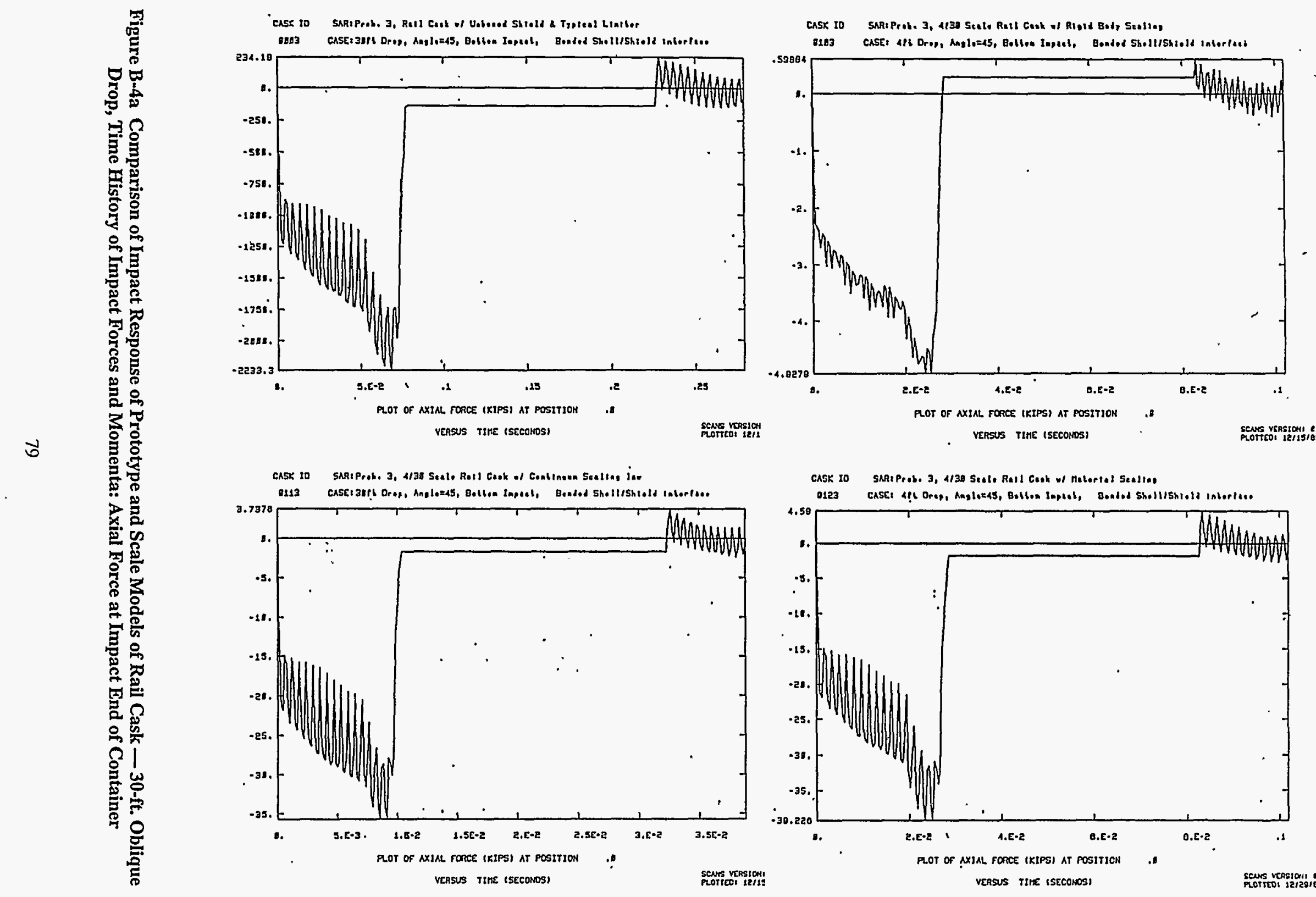

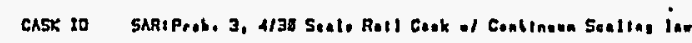

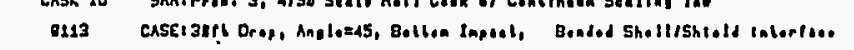

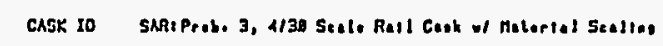

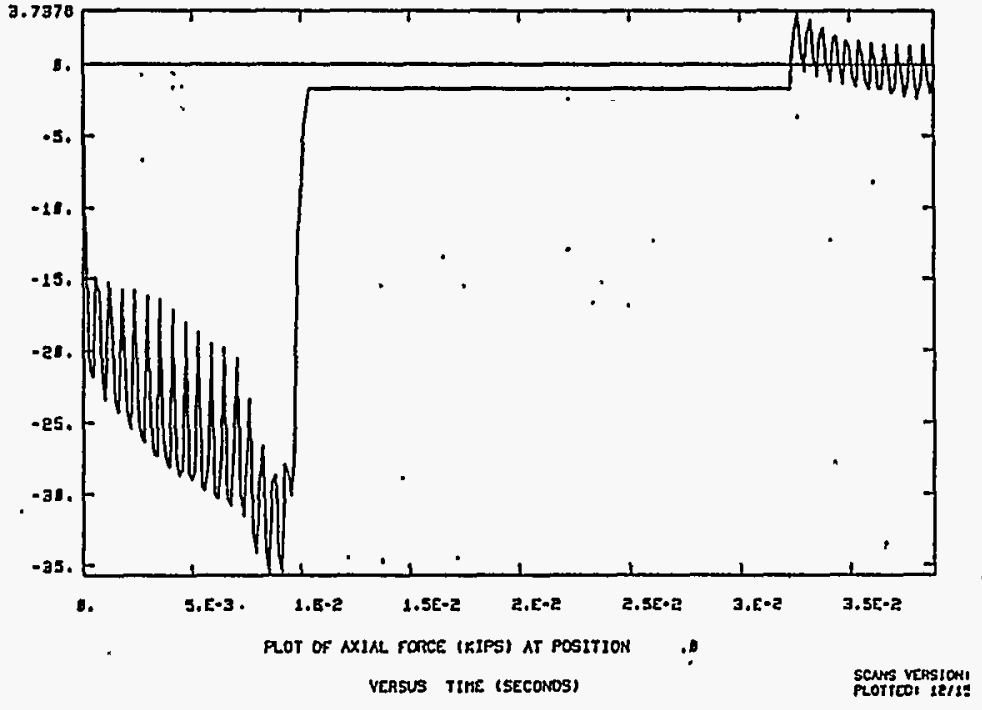

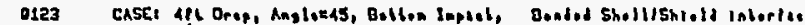

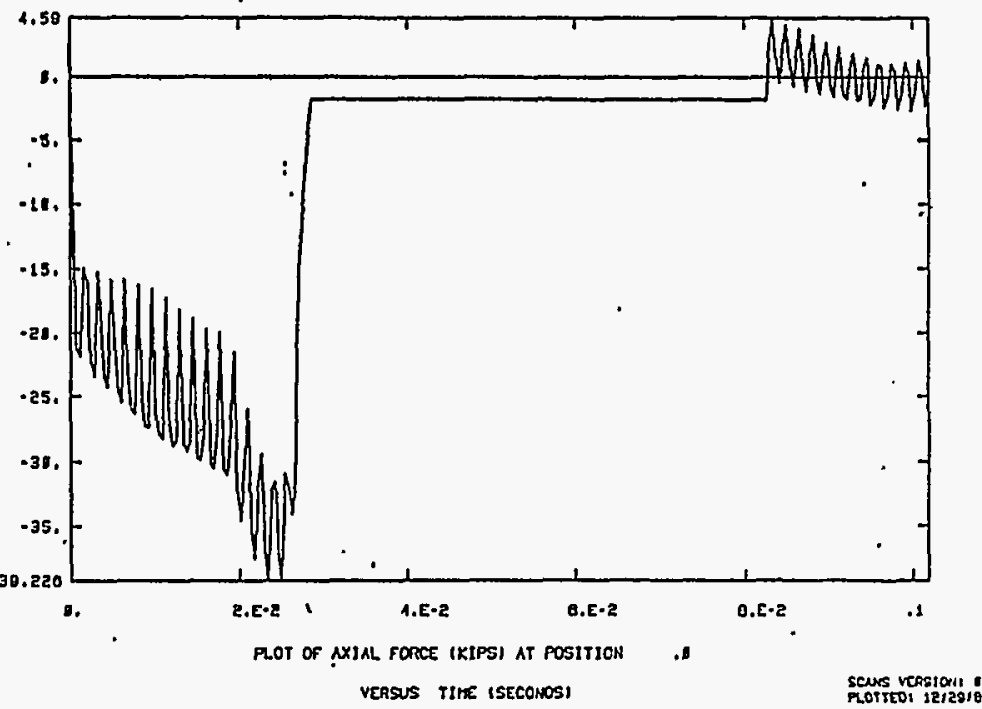




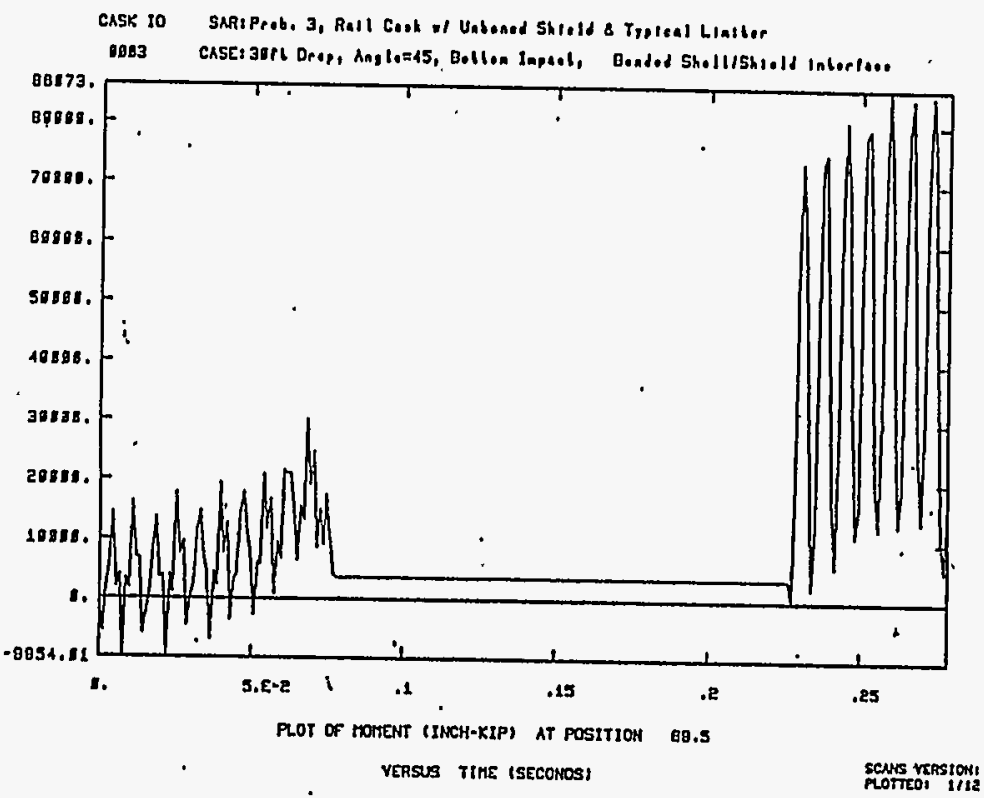

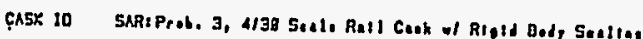

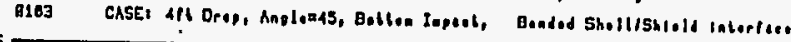
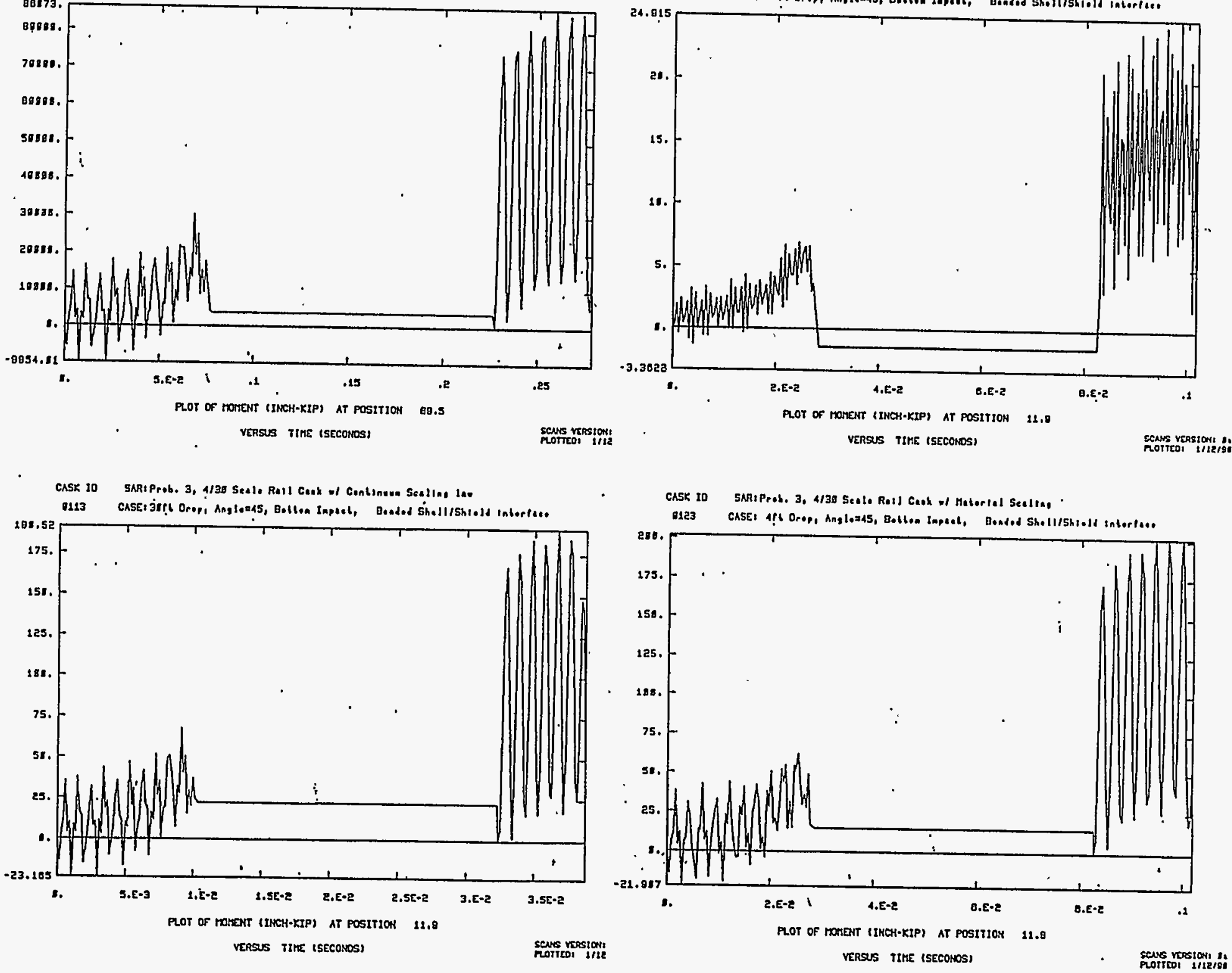


\section{APPENDIX C: INSTRUMENTED MEASUREMENTS}

\section{C.1 Introduction}

Continuous monitoring of accelerations, strains, relative displacements, or pressures at selected locations on a package during a drop test can provide significant insight into the package behavior. Unfortunately, these transient response measurements are not easy to obtain; they require special instruments and equipment. To facilitate this task, this appendix attempts to identify some common requirements, problems, and possible solutions for all instrumented measurements. The appendix begins with a general discussion on topics applicable to all instrumented measurements: instrument setup, instrument characteristics, noise control, and recommended practices. The appendix continues with a description of the characteristics and usage of some common instruments for instrumented measurements in impact testing: accelerometers, strain gages, linear variable differential transformers (LVDT), and filters. The appendix concludes with a discussion on the use of filters for the elimination of noise in recorded and digitized signals and for the analysis and separation of recorded impact responses.

\section{C.2 General Requirements}

\section{C.2.1 Instrument Setup}

Figure C- 1 depicts a typical equipment setup and data flow chart for the measurement of transient response. The procedure, which is basically an electrical signal processing operation, can be divided into two stages, namely, the measurement chain and the analysis chain. The measurement operation is usually carried out using analog (continuous) electrical signals; the analysis operation is executed using digital (discrete) signals.

The equipment used for the measurement can be divided by function into five groups:

- Transducer, which converts the change of a mechanical or thermal response (displacement, velocity, acceleration, force, pressure, or temperature, etc.) into the change of an electrical parameter (resistance, voltage, charge, inductance, or capacitance). The transducer may also have an electrical bridge circuit that converts the change in the electrical parameters into a change in electrical voltage or current, which is the output of the transducer.

- Transmitter, which is an electronic device or simply an electrical conductor used to deliver the electrical output or signal from the transducer to an electrical signal conditioner or recorder. In the past, most drop tests of shipping packages used direct transmission through an electrical wire.

- Signal conditioner, which is an electrical device or circuit to amplify, filter, integrate, differentiate, or perform other simple processing of the electrical signal.

- Recorder, which is an electrical-mechanical equipment to record and replay the processed or unprocessed electrical signal.

- Analyzer, which is a package of electronic circuits and devices, computer hardware and software used to perform special analyses of electrical signals. An analyzer usually can perform a wide range of functions including the filtering, sampling, analog-todigital conversion, storage, Fourier analysis, and graphical presentation of the data. A spectrum analyzer is an effective tool for troubleshooting. The analyzer can decompose an electrical signal into sinusoidal components of various frequencies. The information on the frequency, amplitude, and phase of these components can provide invaluable insight into the cause and nature of the analyzed signal.

Among these five groups of instruments, the transducer has the most direct impact on the measurement. Therefore, subsequent subsections (Subsections C.3-C.5) will provide further information on several frequently used 
transducers for impact tests, namely, the accelerometer, the strain gage and the LVDT relative-displacement gage. These transducers are also good examples for illustration, because they operate on different physical principles and have very different practical problems.

\section{C.2.2 Instrument Characteristics}

Although the various instruments and equipment described in the preceding subsection (Subsection C.2.1) have widely different designs and functions, selection can be based on several common characteristics: stability, consistency, resolution, sensitivity; linearity, frequency range, impedance, and noise.

The stability of an instrument or equipment refers to the susceptibility of its performance to environmental changes. For example, the electrical output from a resistance wire strain gage is sensitive to temperature changes. The performance of many other electronic devices is also affected significantly by temperature. However, since an impact test lasts for a very short duration, the stability of equipment would not be a major concern. A calibration adjustment or check of the instruments before and after each test should be able to keep all stability problems under control. However, the permissible range of operating environmental conditions for all instruments should always be observed, because an instrument may not be accurate or linear in its response outside the range.

The consistency or repeatability of an instrument or equipment refers to the ability of the equipment to produce the same result under identical conditions. The lack of consistency can be caused by mechanical effects such as hysteresis, or by drift effects in the electronics.

For example, a strain gage may produce inconsistent results due to poor bonding between the metal wire and its backing.

The resolution of an instrument or equipment refers to the smallest quantity which the instrument can measure or distinguish. Wirewound devices tend to suffer resolution problems due to sudden change of output from wire to wire. However, most modern transducers produce smooth, continuous output. Thus they can have infinite resolution if not limited by their consistency.

The sensitivity or amplification of an instrument or equipment refers to the ratio of its output to input. A sensitive instrument produces large output from a small input. A larger output is always desirable, because it eliminates the need for amplifiers.

The linearity of an instrument-or equipment refers to the linear relationship between the input and output of the instrument. A linear input-output relation significantly facilitates the data analysis, because the output is simply proportional to the input; no calculation is required. In the specifications of an instrument, the maximum deviation from linearity is usually given as a percentage of the full amplitude range of the instrument. Thus, when the amplitude of a measurement is significantly less than the full range of the instrument, the nonlinearity of the instrument can cause a significantly larger error in the measurement than the maximum deviation percentage indicates.

The frequency range of an instrument refers to the range of frequencies in which the same (linear) input-output relationship holds. Unfortunately, mechanical and electrical systems have natural vibrations. The vibration and resonance in an instrument can cause the instrument to respond differentiy to the same input at different frequencies. Thus, the usable frequency range of the selected instrument may be limited. For example, the frequency range of an accelerometer has a lower limit of several hertz to a high limit of one-fifth of the mounted resonant frequency of the accelerometer. To obtain accurate results, the instrument frequency range should adequately cover the frequencies of the package response to be measured. The information given in Subsection 2.1.2 concerning the dominant frequencies of the three impact responses of a package should be used for this purpose. 
An instrument can have both mechanical and electrical impedances. The mechanical impedance (or dynamic mass) is the resistance of the instrument to mechanical motion. Similarly, the electrical impedance is the resistance of the instrument to an electrical current. Both impedances can. play an important role in the measurement operation. In the case of a transducer mounted on a specimen, a large mechanical impedance of the transducer relative to the local impedance of the test model may affect the model motion which the transducer is supposed to measure. Similarly, in connecting two electrical circuits or instruments together, the electrical impedances of the two circuits should be compatible to ensure that the receiving circuit can accept, without appreciable modification, the output of the delivering circuit.

The mechanical and electrical impedances are frequency-dependent due to the natural vibration characteristics of mechanical and electrical systems. Both the nature and magnitude of the impedances may vary with the frequency. This frequency dependence can be observed in the mechanical impedance or resistance of an accelerometer to the motion of the model at the location on which the accelerometer is mounted. At frequencies considerably lower than its mounted resonant frequency, the accelerometer would behave like a small rigid mass, whereas at frequencies higher than the resonant frequency, the accelerometer would behave like a damper. The impedance of an accelerometer reaches the . maximum value at the resonant frequency.

The noise from an instrument or equipment is the unwanted or meaningless information or distortion that the instrument introduces into the measurement signal. Instrument noise is unavoidable and has many causes. Design limitations of the instrument are usually responsible. All electrical circuits have background noise when the circuit heats up. A filter can distort a signal because of its imperfect frequency response characteristics. A transducer can generate noise because of its sensitivity to environmental conditions or to cross-axis (or transverse) excitations. A tape recording can have noise because of the finite size of the recording head. Similarly, a digitized signal can have noise because of the finite number of bits used to store the information. Obviously, the amplitude of the noise should be kept very small compared to the measurement signal in order to maintain adequate accuracy in the measurements.

In summary, instruments selected for impact tests should have the following characteristics:

- Adequate stability, consistency, resolution, sensitivity and linearity to ensure desired accuracy in the measurement. These characteristics are usually interrelated. The improvement of one would bring similar improvements in others.

- Sufficiently wide frequency range to cover the frequencies of the package response to be measured.

- Compatible or matching impedance with related instruments or package components to minimize the effect of instrument impedance on the measurement signal and the motion to be measured.

- A high signal-to-noise $(\mathrm{S} / \mathrm{N})$ ratio to minimize the effect of instrument noise on the measurement signal.

\section{C.2.3 Noise Control}

Instrumented measurements are prone to noise problems. Noise is generated not only within the instruments as described in Subsection C.2.2, but without and among the instruments in the measurement chain. In general, noise can come from the following sources:

- Individual instrument, equipment, or power source used for the measurement

- Vibrations, and other environmental conditions in the test model and the measurement equipment

- Electromagnetic interaction with other equipment used or not used for the 
measurement, including the Earth's magnetic field

Noise from the first source group can be effectively controlled by selecting instruments with desirable characteristics, as discussed in Subsection C.2.2.

Noise from the second and third source groups can be controlled by relocating, reorienting, or isolating the instruments from the noisy environments. The isolation involves decoupling the instruments from their noisy environments or suppressing the undesirable influence of the environments.

- Mechanical coupling can be minimized using vibration isolators.

- Excessive vibration can be suppressed using vibration dampers.

- Thermal coupling can be reduced by thermal insulators.

- Excessive temperatures can be reduced by cooling.

- Electrical impedance coupling can be avoided by connecting each instrument to a common ground directly, rather than through a.common electric conductor.

- Electric field coupling can be diminished by shielding each instrument using a grounded electrical conductor.

- Magnetic fields cannot be practically shielded, because few special materials are available for the purpose. Instead, electrical current loops which can interact with magnetic fields should be eliminated.

- A wire loop can be eliminated by using a twisted-pair cable. (As discussed in Section C.4, a twisted-pair cable can also be used to solve temperature sensitivity problem.)

- A ground loop (where the ground serves as a conductor to complete the current loop) can be eliminated by connecting all instruments or circuits to a common ground.
Ideally, each electrical circuit has its own shielding system, which is connected directly (instead of through a common conductor) to a common ground with other circuits.

Some special methods have been used to reduce noise in unique situations. In the case where two signals have identical but opposite noise, the noise can be eliminated by superimposing the two signals. Methods based on similar principle are widely used in transducers to rid their measurements of environmental effects. Some temperature-compensated strain gages use the methods to eliminate the influence of temperature on the gage output. Some accelerometers use the methods to eliminate sensitivity to rotational motion. In impact tests, the methods have been used to eliminate unwanted vibration response in the measurement. The application of the methods, however, requires exact information on the relationship among the noise and signals. Subsection C. 4 details some applications of the methods to strain measurements.

A good test plan should identify the potential noise sources and specify methods for their control. The plan should also define calibration procedures to confirm the effectiveness of the noise control measures and to obtain magnitude and frequency information on the remaining noise. Some of the methods are mentioned in Subsection C.2.4.

\section{C.2.4 Recommended Practices}

Properly selecting an instrument requires estimates of the magnitude and frequency range of the package response to be measured. Inadequate estimates can result in large measurement errors and even data losses. To minimize these problems and their effect on the test program, the following practices are suggested:

- Test the signal triggering technique (if used) to ensure its adequacy and reliability

- Take more measurements than needed in each test; have few duplicate or similar measurements to show possible scatter of data 
- Vary transducers and locations for the same measurement

- Make "double" measurements (i.e., recording the same signal using two different range settings for the magnitude and for the time, if necessary)

- Perform zero-input tests (calibration) to measure the level of background noise in the measurement chain

- Perform calibration or verification checks before and after each impact test to detect malfunction of equipment

- Compare related measurements to detect inconsistencies, which indicate possible errors

- Perform Fourier spectrum analysis of measured signal to detect noise and to understand the nature of the measured signal

\section{C.3 Acceleration Measurement}

Acceleration is the most common measurement made in package impact tests. The acceleration measurement can provide the following information on the quasi-static and vibration responses of the package:

- The rigid-body impact acceleration (g load) or impact force on the package;

- The impact duration or the dominant frequency of the quasi-static response;

- The vibration amplitudes and frequencies of the package;

- The relative importance of vibration and quasi-static responses at the measurement location.

An accelerometer is a transducer used to measure absolute accelerations. To measure the acceleration of a location, the base of an accelerometer needs to be mounted to the location with its axis oriented towards the direction of the acceleration to be measured. A typical accelerometer has two basic components: a mass and a load cell (or force transducer). The load cell measures the inertial force of the mass. Figure C-2 depicts a typical accelerometer. To function properly, the mounted resonant vibration frequency of the accelerometer has to be higher than the frequencies of the acceleration to be measured, so that under the excitation of the acceleration, the accelerometer would behave like a rigid mass having the same acceleration as the input. Thus, the inertial force of the accelerometer mass is proportional to the acceleration to be measured, and the output of the load cell is the output of the accelerometer.

The load cell employs either a piezoresistive or a piezoelectric material to measure the inertial force. Under load, the piezoresistive material experiences a change in electrical resistance while the piezoelectric material produces an electrical charge. By measuring these electrical parameters, the inertial load or the acceleration is determined. The piezoresistive accelerometer offers the advantage of $D C$ or static $(0 \mathrm{~Hz}$.) response, which is needed for measuring the long-duration (quasi-static) response of a package in a drop test. The piezoelectric accelerometer responds only to signals with frequencies higher than several $\mathrm{Hz}$. because of amplifier limitations.

At high frequencies, the response of an accelerometer is limited by the resonant vibration of the accelerometer. Most accelerometers exhibit an undamped singledegree-of-freedom vibration response when securely mounted, and the manufacturer usually specifies the mounted resonant frequency of an accelerometer. The response of an accelerometer is normally flat (within $\pm 5 \%$ ) up to about one-fifth of the mounted resonant frequency. Figure C-3 depicts a typical vibration response curve of an undamped accelerometer. The frequency response characteristics of an accelerometer used for the impact test should be calibrated over the entire operating frequency range. Calibrations at $0 \mathrm{~Hz}$ (static calibration) can be accomplished using a centrifuge and at other frequencies using a shake table. 
Subsection C. 2.2 has pointed out that an accelerometer can impede the motion of the test model. The impedance can significantly alter the model response to be measured. This problem usually occurs when the accelerometer is mounted on a flexible location such as a thin panel of the test model. To avoid this problem, a lighter-weight accelerometer should be used. In general, the mass of the accelerometer should be kept less than 5\% of the effective local vibrating mass of the test model at the location. In addition, the mass of the accelerometer should be sufficiently small that a contact resonance between the accelerometer mass and the local stiffness of the test model at the mounting location would not occur at a frequency lower than the mounted resonant frequency of the accelerometer.

\section{C.4 Surface Strain Measurement}

Strains or stresses are often measured in package impact tests to demonstrate the structural integrity of the tested package. By comparing the measured stress to the yield stress of the material and the buckling stress of the structure, the available margin for protection against permanent deformation and buckling can be demonstrated, respectively. Similarly, by comparing the measured strain to the rupture strain (or elongation) of the material, the available margin for protection against rupture can be assessed. (A crude estimate of the permanent strain for this purpose can also be obtained by destructive metallographic examination of the damaged test model if the model material has an identifiable microstructure.) Strain measurements can also show the propagation of stress waves in the package but acceleration measurements usually cannot. From the strain measurement, the following specific information on the impact response including stress waves, can be derived:

- The impact duration of the package;

- The maximum stress, and strain (elastic and plastic) at the monitored locations of the package;

- The vibration stresses and frequencies of the package;
- The amplitude and speed of stress waves in the package;

- The relative importance of all impact responses (waves, vibrations, and quasi-static deformations) at the monitored locations;

- An estimate of the dynamic amplification factor to be used in quasi-static impact analysis of the package.

A strain gage is a transducer used to measure the strain or the relative displacement between two locations on the surface of a test specimen. Three groups of strain gages are commercially available for different applications, namely, the metal-wire or foil gage, the semi-conductor gage, and the capacitance gage. The electrical resistance of the metal wire and semi-conductor gage and the capacitance of the capacitance gage increase proportionally with increasing strain of the gages. The capacitance gage is usually used at high temperatures (above $500^{\circ} \mathrm{F}$ ) while the metal-wire and semi-conductor gages are used at low temperatures $\left(-450^{\circ} \mathrm{F}\right.$ to $500^{\circ} \mathrm{F}$ ). The semi-conductor gage has significantly higher sensitivity and higher cost than the wire gage. The gage factor (i.e., the ratio of resistance change to strain) of the semiconductor gage is about 50 as compared to 2 in the wire gage. Despite its low sensitivity, the metal wire or foil gage is adequate for general applications and has been used in many package impact tests in the past. Figure C-4 depicts several metal wire and foil gage designs.

Most strain gages have negligible mass. Therefore, they do not encounter the same problems of limited frequency response range and mismatched mechanical impedance as the accelerometer. As a result, most strain gages have very wide frequency response ranges $(0$ $\mathrm{Hz}$ to greater than $50 \mathrm{KHz}$ ) and should be able to measure the rapid change of strain at a wave front. In this case, the gage length usually limits the accuracy of the measurement.

Since a strain gage measures the average strain over the gage length, the measurement is exact only in the case where the test model has 
uniform strain within the gage length. A strain gradient can introduce an error in the measurement, and a sufficiently short gage length should be used to control the error. For this reason, a shorter gage length should be used for measuring the strain of a higher frequency and at a location with greater stress concentration. The highest frequency of the test model response should be used to select the gage length. For example, if a strain gage is used to measure strain generated by a stress wave front, and the variation of strain within the gage length is to be kept less than $\pm 1 \%$ of the amplitude of the wave, then the strain gage length used for the measurement should not be greater than $1 / 50$ the thickness of the wave front. Assuming the wave front has a rise time of $0.1 \mathrm{msec}$ and a speed of $200,000 \mathrm{in} / \mathrm{sec}$ (longitudinal elastic wave in steel), the gage length should not be greater than $2 / 5$ inches (i.e., $0.0001 \times 200000 / 50$ ). The limit for a slower wave would be smaller. However, the gage length is usually not a limiting factor in the impact test of packages.

One major source of errors for the resistance strain gage is its temperature sensitivity. Temperatures can change the gage factor, the gage length and the gage electrical resistance. The change of gage factor is minimal within the operating temperature range of the gage. The change in gage length is not significant unless the gage and the test specimen have different thermal-expansion coefficients. The difference in thermal expansions between the strain gage and test specimen can result in an artificial or apparent strain. The change of gage resistance caused by the temperature can be as large as the change by the strain. Considering a typical metal wire strain gage having a gage factor of 2 , and a temperature coefficient of resistance (change) of $1.6 \times 10^{-5} /{ }^{\circ} \mathrm{F}$, the change of resistance produced by a temperature change of $1^{\circ} \mathrm{F}$ is as large as the resistance change generated by the elastic deformation of steels under a stress of $250 \mathrm{psi}$ (i.e., $30 \times 10^{-6} \times 1.6 \mathrm{x}$ $10^{-5} / 2$ ).

The temperature effects on strain gage can be minimized by counteracting the effect of resistance change with the effect of thermal expansion difference. By selecting a gage material with proper thermal expansion and temperature coefficient of resistance, a selftemperature compensated (STC) gage can be produced for a specific test specimen material. The STC gage will exhibit minimal apparent strain over a range of temperatures, if it is used on a test specimen made of the specified material. A more general approach to overcoming the temperature sensitivity difficulty is to use more than one active gage exposed to the same temperature and strain environments. By properly combining the outputs of these gages, the unwanted temperature effects can be eliminated by cancellation. The combination of outputs is usually carried out within the measurement circuit, the Wheatstone bridge.

Figure C-5 depicts the classical Wheatstone bridge measurement circuit for resistors, which is commonly used for measuring the small resistance change-of-strain gages. If the resistance of the resistors (R1, R2, R3, and R4) in the circuit satisfy the condition R1/R4 = $R 2 / R 3$, then the output voltage of the circuit $\left(V_{\text {out }}\right)$ vanishes and the bridge is balanced. $A$ change in the resistance of one of the resistors (e.g., R1) will unbalance the bridge and produce a voltage output. If the resistance change is not large and the circuit is not significantly out of balance, the voltage output will be proportional to the resistance change.

In the Wheatstone circuit, if a similar change occurs in two adjacent resistors (e.g., R1 and R4, or R2 and R3), the bridge will remain balanced and the output voltage will be zero. However, if the changes are opposite (i.e., if R1 increases and $\mathrm{R} 4$ decreases), the voltage output will be doubled. This result suggests that the outputs of strain gages can be canceled or amplified by placing the strain gages at different locations in the measurement circuit. This method is frequently used to minimize noise and temperature effects on strain gage measurements. Figure C-6 depicts the different arrangements of the bridge circuit used for strain gage measurements. The full and half 
bridges can be used to eliminate temperature effects. However, the quarter bridge should be used only with a self-temperature compensated (STC) strain gage. The full bridge is used in many accelerometers for measuring the inertial force or the acceleration.

By depicting the lead wire in various lengths, Figure C- 6 brings to attention the importance of the length of the lead wires in the measurement circuit. A long wire can have a significant resistance and resistance change in comparison to those of the strain gage. Therefore, a long lead wire should be counter-balanced with an adjacent wire of identical length and properties. Moreover, a twisted-pair cable should be used for the two lead wires to keep the wires in the same environment conditions and to avoid the formation of an electric current loop, which can interact with a magnetic field to produce noise as discussed in Subsection C.2.5.

To increase the signal-to-noise ratio in the strain gage measurement, increased output from a strain gage is necessary and can be achieved by using a higher gage resistance (which allows a higher input voltage to be used for the measurement circuit) or using more than one active strain gage (i.e., using a half or full bridge) in the measurement circuit.

For low-temperature application, strain gages are installed on the surface of the test model using epoxy or other polymeric adhesives. The thickness and properties of the adhesive can affect the transmission of the model strain to the gage. The relation between the strain gage output and the model strain cannot be truly calibrated without installing the gage on the test model. However, a known force or strain is often impossible to apply to the test model for the necessary calibration. Therefore, the common calibration of strain gages is done by electrical simulation (i.e., a shunt calibration). Either the voltage/current output or the resistance change of a strain gage in response to an applied force/strain is simulated and used to calibrate the measurement circuit.

Unfortunately, this calibration verifies only the electrical performance of the strain gage circuit but not the mechanical performance of the installed gage. Thus the quality of the installation of a strain gage is not verified. In view of the importance of the installation to the gage performance, a good test plan should require that the method of installation be precisely specified in the test procedure and the method and the installer be properly qualified for the task. The necessary qualification can be accomplished by installing a strain gage to a test specimen, to which a known force or strain can be applied for the calibration. Of course, the calibration specimen and the test model should be under similar conditions, which include the temperature, material, surface geometry, roughness, and strain.

\section{C.5 Relative Displacement Measurement}

A limit on the maximum relative displacement between two adjacent components of à package may be needed to maintain the containment, subcriticality, or shielding of the radioactive contents of a shipping package. If such a limit can be defined by appropriate analysis or other means, the displacement can be measured in an impact test to demonstrate the margin of safety of the package design.

\section{Linear Variable Differential Transformers} (LVDT) are commercially available for measuring displacement. Figure $\mathrm{C}-7$ shows the geometric design, electrical circuit, and output of a typical LVDT. The transducer has two parts, a core in the form of a long solid circular rod and an outer case in the form of a thickwalled circular cylinder. The core fits and slides freely in the center cavity of the outer case. To measure the relative displacement between two locations, the core is attached to one of the locations and the outer case to the other.

The outer case of the LVDT contains a primary coil and a secondary coil. When an alternating electrical current of constant voltage and frequency is applied to the primary coil, it induces an alternating current of the same frequency but different voltage in the secondary coil. The axial position of the core relative to the outer case determines the magnitude of the induced voltage. Within a 
limited range of displacements of the core, the induced voltage varies linearly with the relative displacement between the core and the outer case. Figure C-7 shows a typical AC output from the secondary coil. This output must be rectified and filtered to obtain the DC output shown in the same figure. For dynamic measurements, the frequency of the $\mathrm{AC}$ current (carrier frequency) must be significantly higher than the highest frequency of the dynamic response. Otherwise, filtering out the carrier signal can introduce an appreciable error in the response signal. To minimize this error, the chosen carrier frequency should be at least 10 times the highest frequency of the response to be measured.

Similar to the accelerometer, the core and outer case of an LVDT possesses appreciable mass.

The same precautions discussed in Subsection C-3 concerning undesirable dynamic interactions between the accelerometer and the test model also apply here.

\section{C.6 Filters and Filtering}

\section{C.6.1 Filters}

Filters are electrical circuits or devices used to eliminate unwanted spectral or Fourier components from an electrical signal. Filters are. used to process instrumented measurements in many ways. They are used to rid the measured signal of spurious components before sampling and digitizing the signal (anti-aliasing filtering). They are also used to separate the measurement into contributions from various sources. In the present case of impact tests on shipping packages, filters are commonly used to obtain the rigid-body acceleration and other information on the quasi-static response of the package. This subsection provides the necessary background for the later discussion by first describing the characteristics of filters and possible problems with their application. Details of these applications are deferred to Subsections C.6.2 and C.6.3.

A filter treats each Fourier (sinusoidal) component of the input signal differently according to the frequency of the component.
The filter either permits a component to pass through with little change in amplitude or restricts the passage by drastically reducing or attenuating the component's amplitude. A filter also modifies the phase in addition to the amplitude of the components. A filter is called a low-pass, a band-pass or a high-pass filter, depending on whether the filter allows the passage of all low-frequency, intermediatefrequency, or high-frequency components, respectively. Fig. C-8 shows the amplitude attenuation and phase shift that a typical lowpass filter can produce at various frequencies. The $\mathrm{n}$ number denotes the order of the filter; $\mathrm{a}$ higher-order filter produces a greater attenuation within the same frequency range.

Among the three groups of filters, the low-pass filter is most frequently used for conditioning and analyzing impact test data. Among low-pass filters, the Butterworth filter, whose frequency response characteristics are shown in Fig. C-8, has found the most acceptance because it has the flattest amplitude response in the low frequency range and thus causes the least amplitude distortion of the filtered signal. Unfortunately, the filter has a nonlinear relationship between the phase shift and the frequency, which would cause the Fourier components of the filtered signal to have different time delays resulting in a time distortion of the signal. To overcome this problem, a digital version of the filter has been developed. The digital filter eliminates the time delay by filtering the signal two times in reversed order of time; the signal arriving first in the first filtering is the last to filter the second time. Thus the gain in time of a Fourier component relative to the others will be lost in the second filtering, and the Fourier components will not have different time delays after the second filtering. Unfortunately, the method introduces other undesirable effects: the filtered signal may exhibit small false signals or noise before the beginning time of the original signal, and the filtered signal may show a different cut-off frequency from the specified cut-off frequency of the filter. 
Figure C-8 shows how an analog Butterworth filter would modify the amplitude and phase angle of a sinusoidal electrical signal of various frequencies. The figure shows that the filter does not appreciably change the amplitude of an input signal in low frequencies until the signal frequency exceeds one half of the cutoff frequency of the filter $\left(f_{c}\right)$. The cut-off frequency is defined for this filter as the frequency at which the attenuation of the amplitude of the input signal reaches $3 \mathrm{~dB}$, where $1 \mathrm{~dB}$ equals 20 times the common logarithm of the amplitude ratio. In other words, the amplitude and power (proportional to the square of the amplitude) of a sinusoidal input at the cut-off frequency will be reduced to .707 and .5 of their original values, respectively. Above the cut-off frequency, the amplitude attenuation increases rapidly with increasing frequency. The maximum attenuation or rolloff rate of the amplitude is $6 \mathrm{n} \mathrm{dB}$ per octave, where $n$ is the order or the number of poles of the filter and the value of an octave equals $\log$ (frequency ratio / 2). Fig. C-8 shows that a second order Butterworth filter $(n=2)$ quadruples its attenuation of the amplitude (12 $\mathrm{dB}$ ) when the frequency is doubled (1 octave).

The use of filters to process electrical signals or instrumented measurements faces two major difficulties: (1) the filter may introduce distortions into the filter signal, and (2) the signal components to be filtered out may be too close in frequency to the components to be retained. The first problem was discussed earlier in this subsection. The second problem will be discussed in Subsections C.6.2 and C.6.3.

\section{C.6.2 Anti-Aliasing Filtering}

Digitizing an analog signal requires sampling the signal at isolated times. The sampling operation can introduce into the sampled data 'alias' frequencies, which are different from the frequency of sampling and from any frequencies of the original data. Figure C-9(a) illustrates the cause for an alias frequency. The figure shows a single-frequency sinusoidal analog signal being sampled at a fixed sampling frequency. The circled data points are the sampled results. As indicated by the dashed line connecting the data points, the sample data also vary sinusoidally with time at a constant frequency which is different from the frequencies of sampling and of the analog signal. This apparent frequency of the sampled data is the alias frequency.

By experimenting with the sampling process in Fig. C-9(a) using a sampling frequency $\left(f_{s}\right)$ slightly greater than, equal to, or slightly less than the signal frequency $\left(f_{m}\right)$, one would discover that the sample frequency produces the same alias frequency regardless of whether it is above or below the signal frequency, and the magnitude of the alias frequency is the absolute difference of the sampling frequency and the signal frequency $\left(I f_{s}-f_{m} l\right)$. The Fourier spectrum in Fig. C-9(b) depicts this result. In general, the Fourier spectrum of the sampled data would show many more alias frequencies at frequencies given by the formula, $l_{ \pm} n f_{s} \pm f_{m} l$, where $\mathrm{n}$ is an integer. The alias frequency obtained here and depicted in Fig. C-9(b) is only the lowest of all alias frequencies generated by the sampling process. Therefore, Figure C-9(c) shows an actual measurement signal which contains many Fourier components of various frequencies as depicted in the Fourier spectrum. If all alias frequencies within the range of 0 to $f_{k}$ are to be eliminated in the sampled data, two actions should be taken: first, a low-pass filter is used to eliminate all Fourier components having a frequency higher than $f_{k}$; secondly, a sampling frequency $\left(f_{s}\right)$ greater than or equal to $2 \times f_{k}$ is used to sample the filtered signal. The result of the first action is that there exist no Fourier components in the filtered signal to interact with the sampling frequency to produce alias frequencies lower than $f_{k}$. The remaining Fourier components after the filtering will not produce alias frequencies lower than $f_{k}$.

Accordingly, within the frequency range from 0 to $f_{k}$, the sampled digital signal contains only the actual Fourier components of the original analog signal. The filtering process before sampling is called the anti-aliasing filtering. It can be accomplished using the Butterworth filter described in Subsection C.6.3. In practice, since filters do not possess perfect cutoff 
characteristics, the cutoff frequency of the filter used for the anti-aliasing filtering has to be greater than $f_{k}$ and the sampling frequency greater than $2 \mathrm{f}_{\mathrm{k}}$.

Choosing the sampling and filtering frequencies requires setting limits on the amplitude attenuation of the retained and discarded signal components. A reasonable goal is to reduce the amplitude of the unwanted components to less than $1 \%$ of its original value (i.e., a reduction greater than $40 \mathrm{~dB}$ ) but to reduce the amplitude of the retained components no more than $1 \%$ of its original value (i.e., a less than $0.09 \mathrm{~dB}$ reduction). The frequencies at which a filter . produces these reductions can be identified in the amplitude frequency response of the filter (Figure C-8). If the frequency above which the filter produces a greater than $0.09 \mathrm{~dB}$ reduction is $f_{c}$, the filter should be chosen to have an $f_{c}{ }^{\prime}$ greater than the highest frequency to be retained in the sampled signal (i.e., $f_{k}$ in the present case). Similarly, if the frequency above which the filter produces a greater than $40 \mathrm{~dB}$ reduction is $f_{c}{ }_{c}$, the sampling should be carried out at a frequency higher than two times $f_{c}$ ". Using a 4th-order $(n=4)$ Butterworth filter as an example, the amplitude response curve of this filter in Figure C-8 indicates that $f_{c}{ }_{c}$ is about .75 $f_{c}$ and $f_{c}$ " is about $3.1 f_{c}$.

Therefore, in order to meet the above-stated filter selection criteria, (1) the filter should have a cutoff frequency $\left(f_{c}\right)$ greater than 1.33 (i.e., 1/.75) times the highest frequency to be retained $\left(f_{k}\right)$, and $(2)$ the sampling frequency $\left(f_{s}\right)$ should be greater than 6.2 (i.e., $2 \times 3.1$ ) times the cutoff frequency or 8.3 (i.e.; $1.33 \mathrm{x}$ 6.2) times the highest retained frequency $\left(f_{k}\right)$. Accordingly, in practice, the sampling frequency $\left(f_{s}\right)$ needs to be much greater than two times the retained frequency $\left(f_{k}\right)$ as suggested by the sampling theory.

\section{C.6.3 Separation of Package Responses by Filtering}

Subsection 2.2 .2 points out that the impact response of a package contains three components, namely, the wave, vibration, and quasi-static deformation responses. These responses affect the package differently and thus have different design requirements. Moreover, depending on the package design and impact condition, any of the three responses can dominate the response and cause damage to the package. Therefore, it is necessary to identify and separate the contributions of these responses in the test measurements. This task can be accomplished using filters, because the three responses usually have different frequencies. In the past, filtering has been used on drop-test measurements for the following purposes:

- To rid the acceleration measurement of the resonant response of the accelerometer, which is usually caused by the wave response

- To identify the dominant vibration frequencies and amplitudes of the package

- To obtain the quasi-static response of the package, which includes the rigid-body acceleration, impact force, etc.

A low-pass filter is used for the first and the third purposes, and a band-pass filter is needed for the second purpose.

Selecting the proper filter for the tasks requires a knowledge of the dominant frequency of the various responses. In general, as discussed in Subsection 2.1.2, the quasi-static response occupies the lowest frequency range which can be estimated using the total duration of the impact. The vibration response is in the intermediate frequency range which is determined by the frequencies of the lower vibration modes of the package responding to the impact. The wave response has the highest frequency range, because of the sharpness of the wave front. Therefore, the quasi-static response can be obtained by using a low-pass filter with a cutoff frequency located between the frequency ranges of the quasi-static and the vibration responses. However, the two frequency ranges need to be sufficiently separated in order to obtain accurate results from this process. For example, using a 4th-order Butterworth low-pass filter and the filter selection criteria specified in Subsection C.6.2 (i.e., the selected filter should reduce the amplitude of the unwanted sinusoidal 
components more than $-40 \mathrm{~dB}$, but the retained components less than $-0.09 \mathrm{~dB}$ ), the lowest vibration frequency should be at least 4.1 times the quasi-static response frequency. This means that if the lowest package vibration is $1000 \mathrm{~Hz}$, the impact duration should not be less than 4.1 msec. (4.1/1000) in order to obtain the rigid body acceleration accurately using a low-pass filter. Most shipping casks of common design meet this requirement; the lowest vibration frequency of the package is between 500 and $1000 \mathrm{~Hz}$. and the impact duration of a $30-\mathrm{ft}$ drop is between 4 and $25 \mathrm{msec}$.

The results obtained by Glass (1989) can be used to demonstrate the adequacy of using filtering to separate impact responses. Figure C10 shows two sets of filtered acceleration histories from the reference. The two sets are obtained from the same acceleration measurement using low pass filters of different cut-off frequencies. The accelerations in the set were measured using two accelerometers (A1 and A2) located at the non-impact end of a circular hollow aluminum (6061-T0) cylinder during a flat $\left( \pm 1^{\circ}\right)$ end-on drop from a height of 30 feet. The cylinder has an inner diameter of 3.58 in., a wall thickness of 0.92 in., and a length of $45.0 \mathrm{in}$. The upper set of acceleration time histories in Fig. C-10 was obtained from the measured data using a digital Butterworth lowpass filter having a cutoff frequency of 10,000 $\mathrm{Hz}$. This filtered time history contains both the quasi-static and vibration responses. The vibration response, however, appears to dominate the result. The frequency of the prevailing oscillations in the histories is close to the lowest (undamped) longitudinal vibration frequency of the cylinder $(2435 \mathrm{~Hz}$, period $=0.41 \mathrm{msec})$. The lower set of time histories shown in the figure was similarly obtained from the same data by using a lower cutoff frequency of $1000 \mathrm{~Hz}$. This set of time histories mainly shows the quasi-static response. Both time histories show a peak acceleration of $2700 \mathrm{~g}$. The same reference [Glass (1989)] has shown that this peak acceleration compares closely to the peak rigidbody acceleration of $2700 \mathrm{~g}$ obtained from an analysis of the impact using the PRONTO computer program.
To demonstrate the use of quasi-static analysis for evaluating impact test results, the rigid-body acceleration obtained from the preceding impact test is used here to estimate the maximum permanent strain near the impact end for comparison with the test measurement. To this end, the average maximum impact stress at a location 3 inches above the impact end can be obtained using the quasi-static analysis method for impacts (i.e., using the peak rigid-body acceleration and the mass of the cylinder above this location). The stress can be found to be $11130 \mathrm{psi}$, which has exceeded the yield stress of 9169 psi and thus would produce a permanent strain. The permanent strain corresponding to this stress can be found from the stress-strain curve to be 6671 microstrain. This value is considerably less than the experimental value of 11600 microstrain. The experimental value, however, is very close to the prediction of 11600 microstrain by the Pronto analysis which included all impact responses. In addition, the post-test measurement of the outer diameters of the cylinder at various axial locations has indicated that the distribution of permanent deformation is not similar to the quasi-static deformation; the permanent deformation has reached an axial distance farther from the impact end than the quasi-static analysis predicts. All these discrepancies between the results of the test and of the quasi-static analysis indicate that in this case the quasi-static response does not dominate the impact response, and the contribution from the wave and vibration responses is appreciable.

If the frequency ranges of the quasi-static and vibration responses, unfortunately, overlap, the quasi-static and vibration responses will be inseparable by simply using a low-pass filter. The contribution of various vibration modes of the package must be evaluated and subtracted from the total response in order to find the quasi-static contribution. This operation will need detailed information on the package vibration frequencies and modes, which can only be obtained with extra efforts by modal tests or analysis. 


\section{- Measurement chain}

Electrical output from transducers mounted on the package

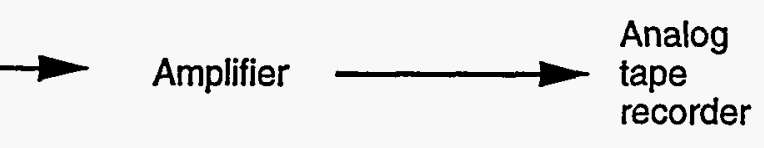

\section{- Analysis chain}

Analog tape with recorded data signals
Signal transmission lines

("Umbilical Cord")
Anti-aliasing filter

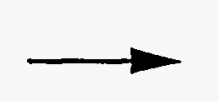

Analog to digital converter

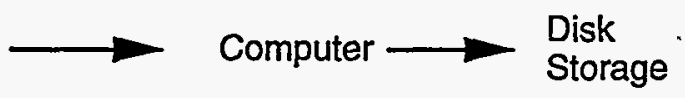

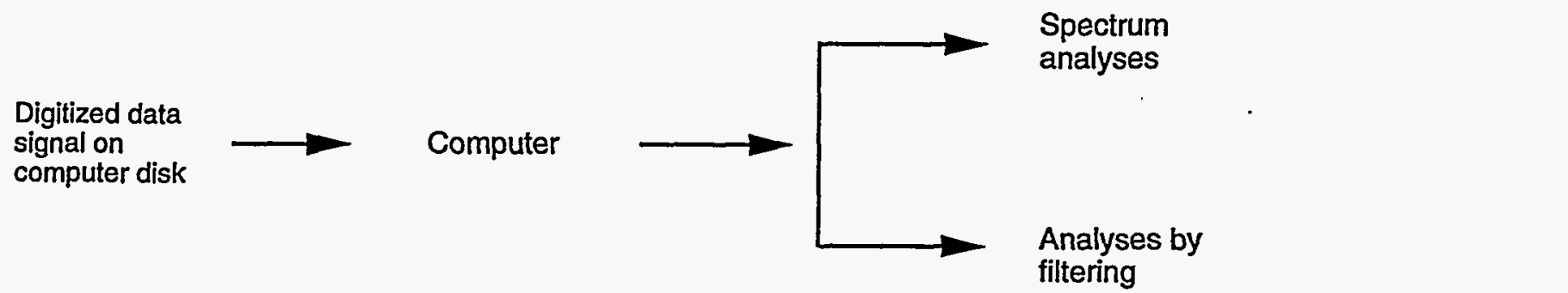

Figure C-1 Instrument Set-up for Transient Measurements 

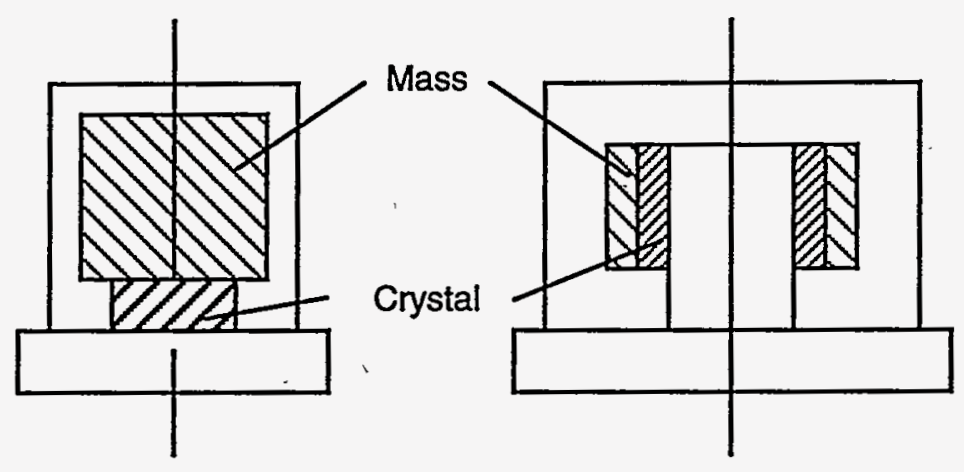

Figure C-2 Typical Design of Accelerometer 


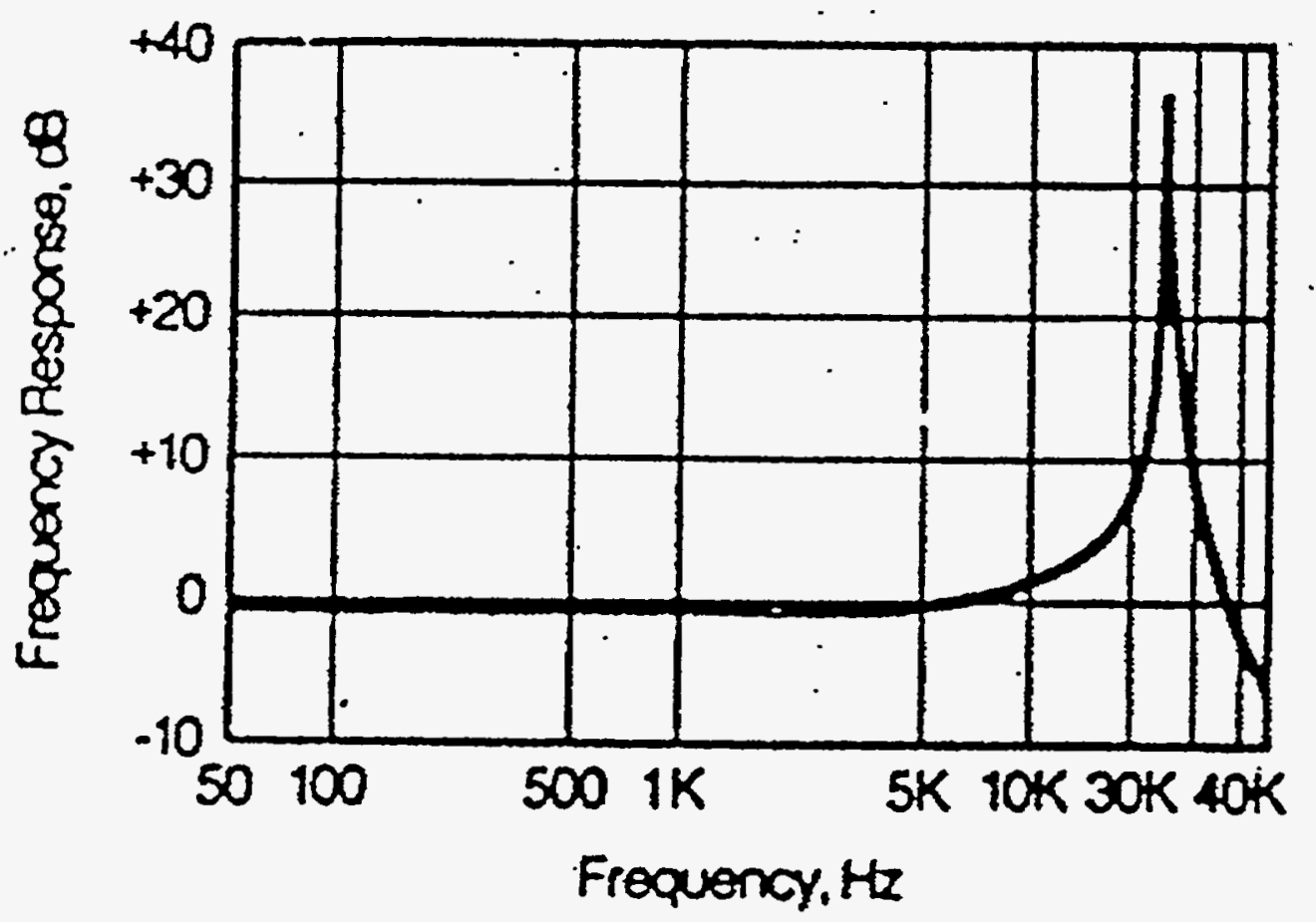

Figure C-3 Typical Frequency Response Curve of Accelerometer 
Appendix C: Instrumented Measurements
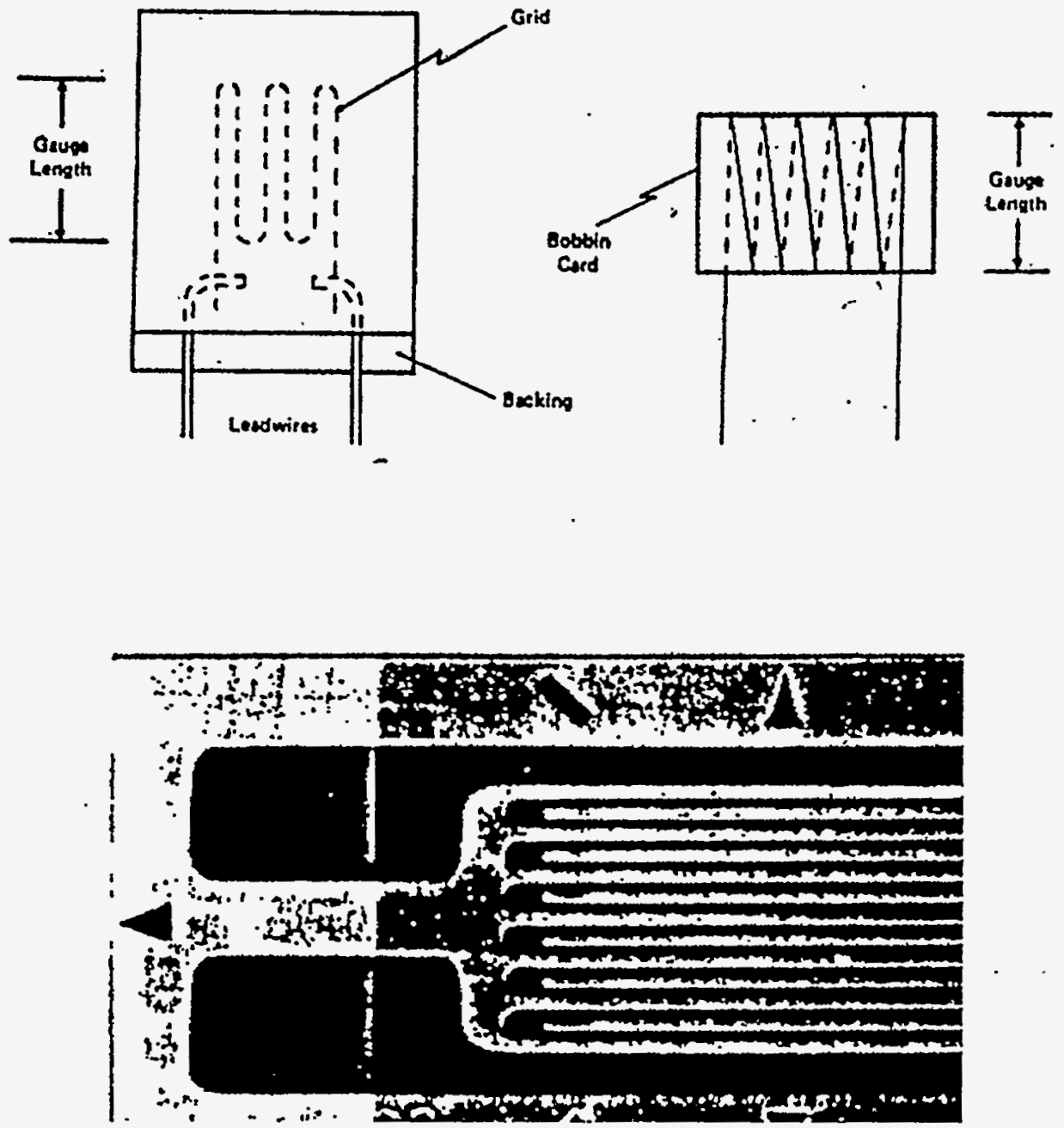

Figure C-4 Typical Design of Wire-Resistance Strain Gage 


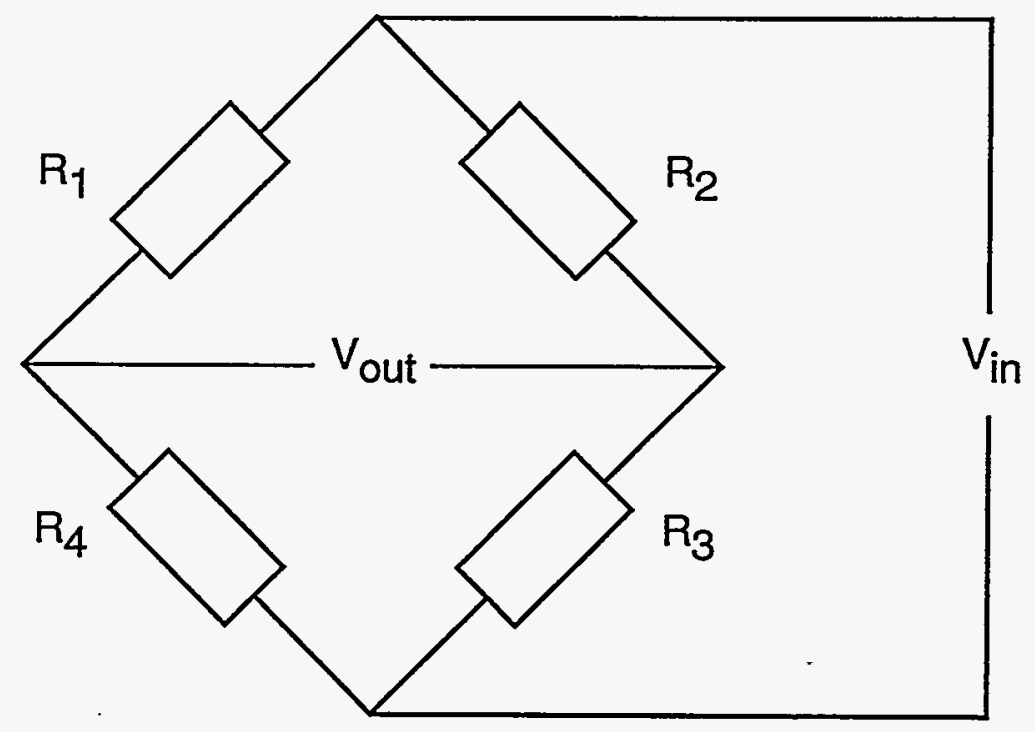

Figure C-5 Wheatstone Bridge Circuits for Measurement of Resistance Change 
Quarter bridge

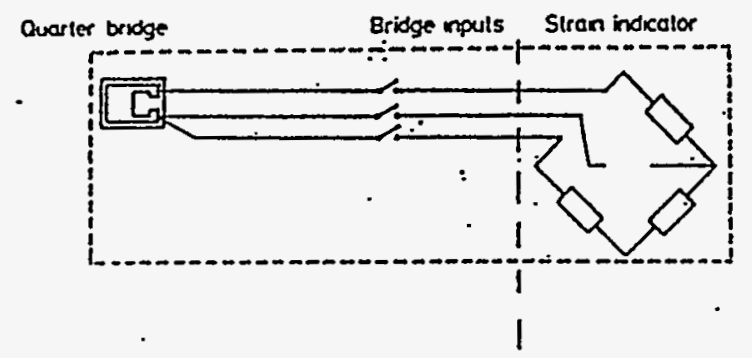

Half bridge

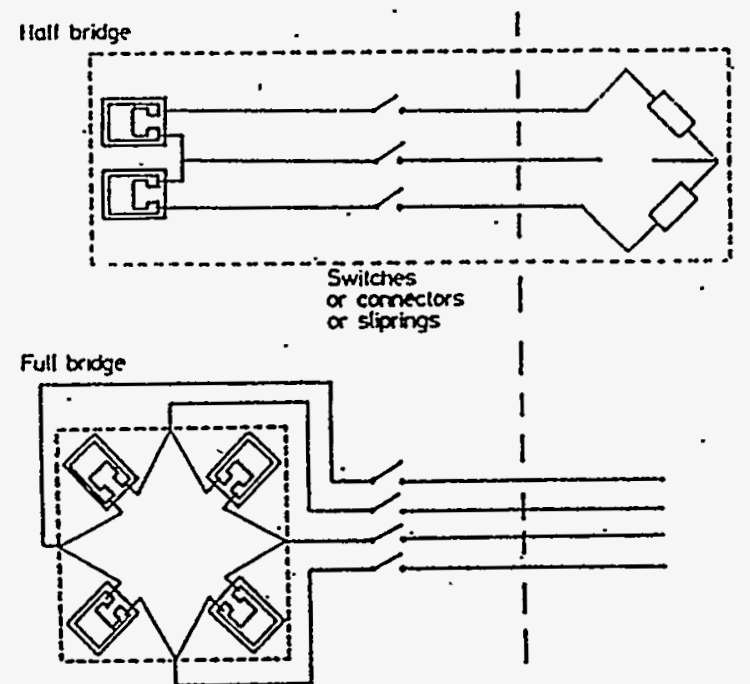

Full bridge

The full bridge circuit is used in many accelerometers.

The quarter bridge should only be used with a temperature-compensated gage.

Figure C-6 Variations of Wheatstone Circuit for Measurement of Strain 

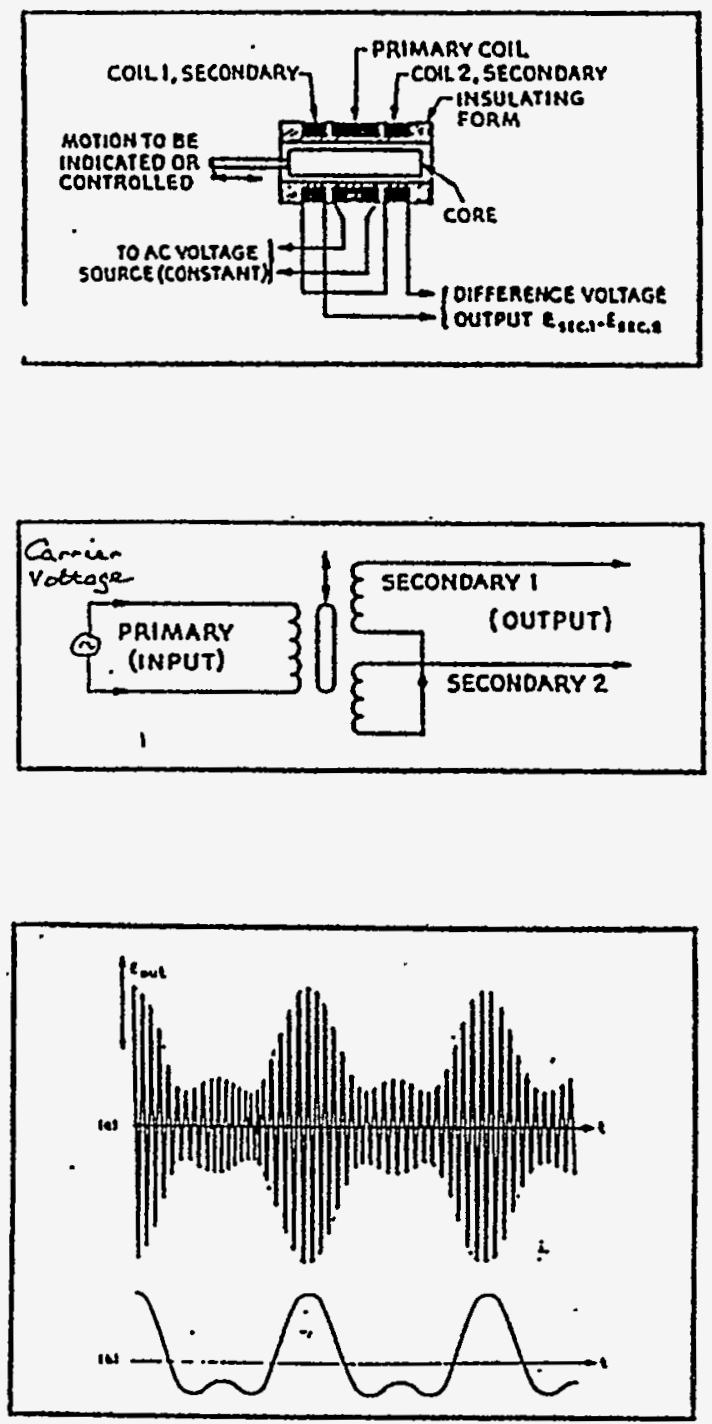

Figure C-7 Typical Design, and Output of Linear Variable Differential Transformer (LVDT) 

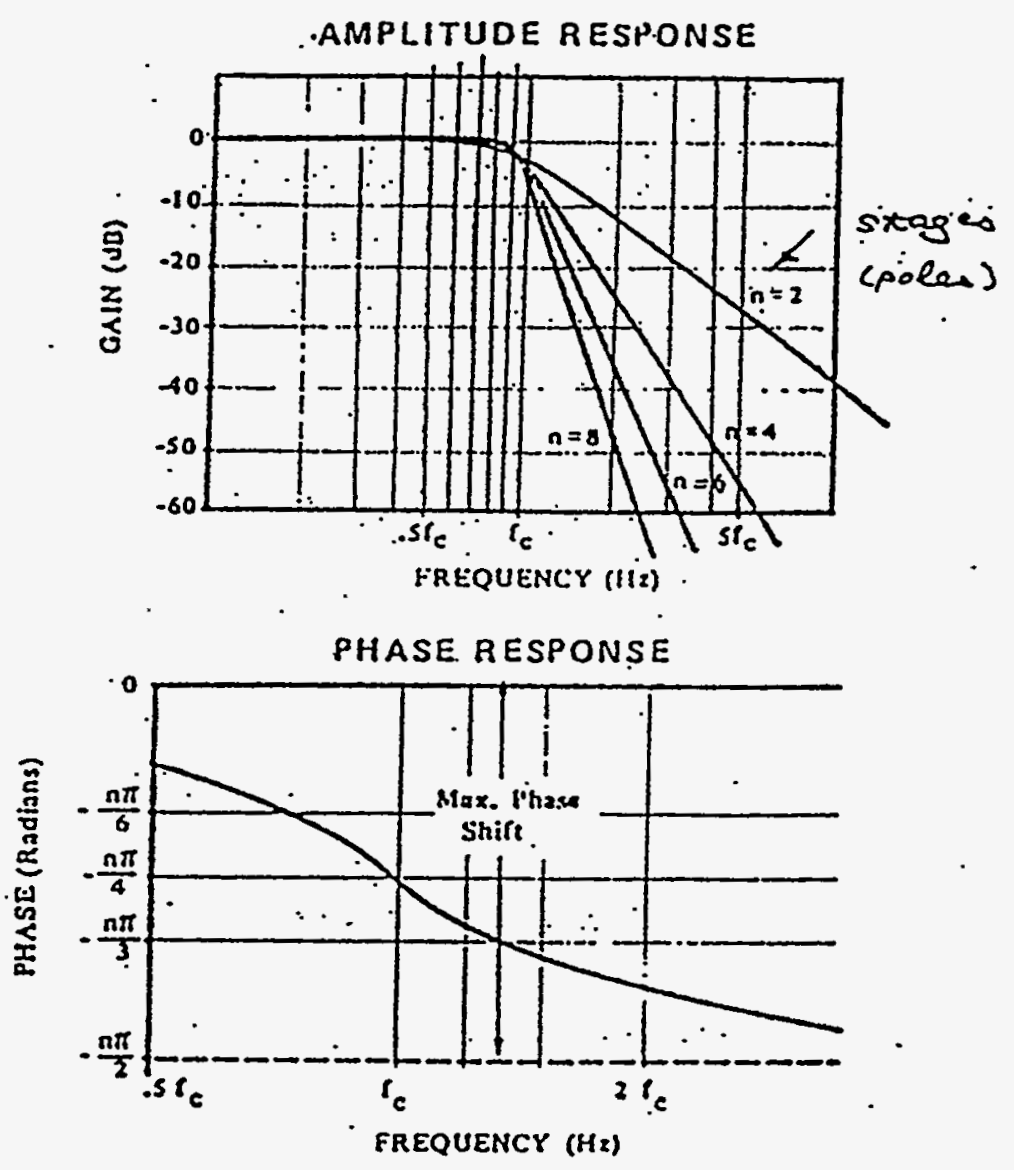

Figure C-8 Frequency Response Characteristics of Butterworth Filter 
(a)

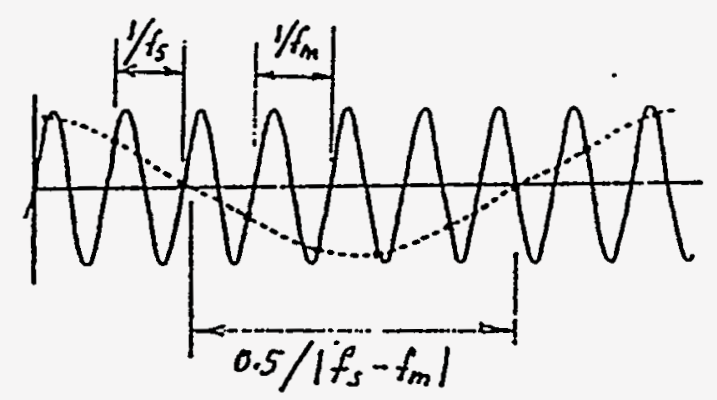

(b).

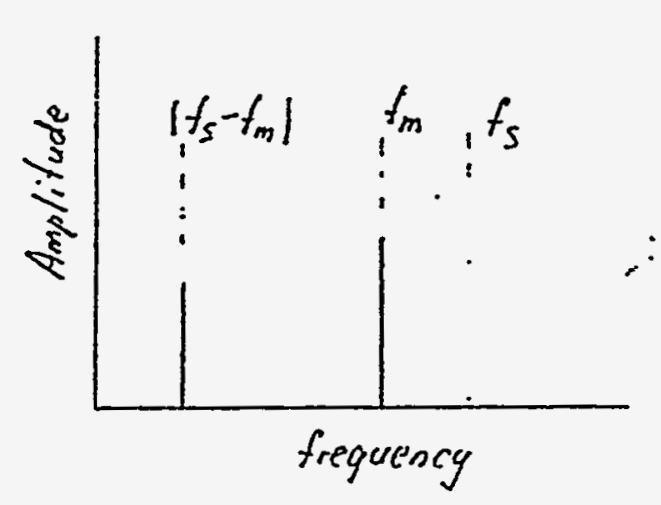

(c)

Frequencies to be

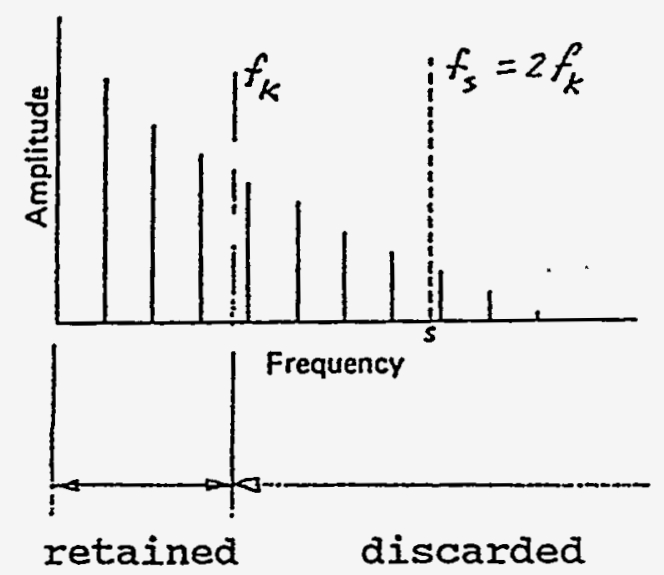

Figure C-9 (a) Sampling of Single-Frequency Analog Signal

(b) Relationship Among Signal, Sampling, and Alias Frequencies

(c) Fourier Spectrum of Multiple-Frequency Analog Signal 

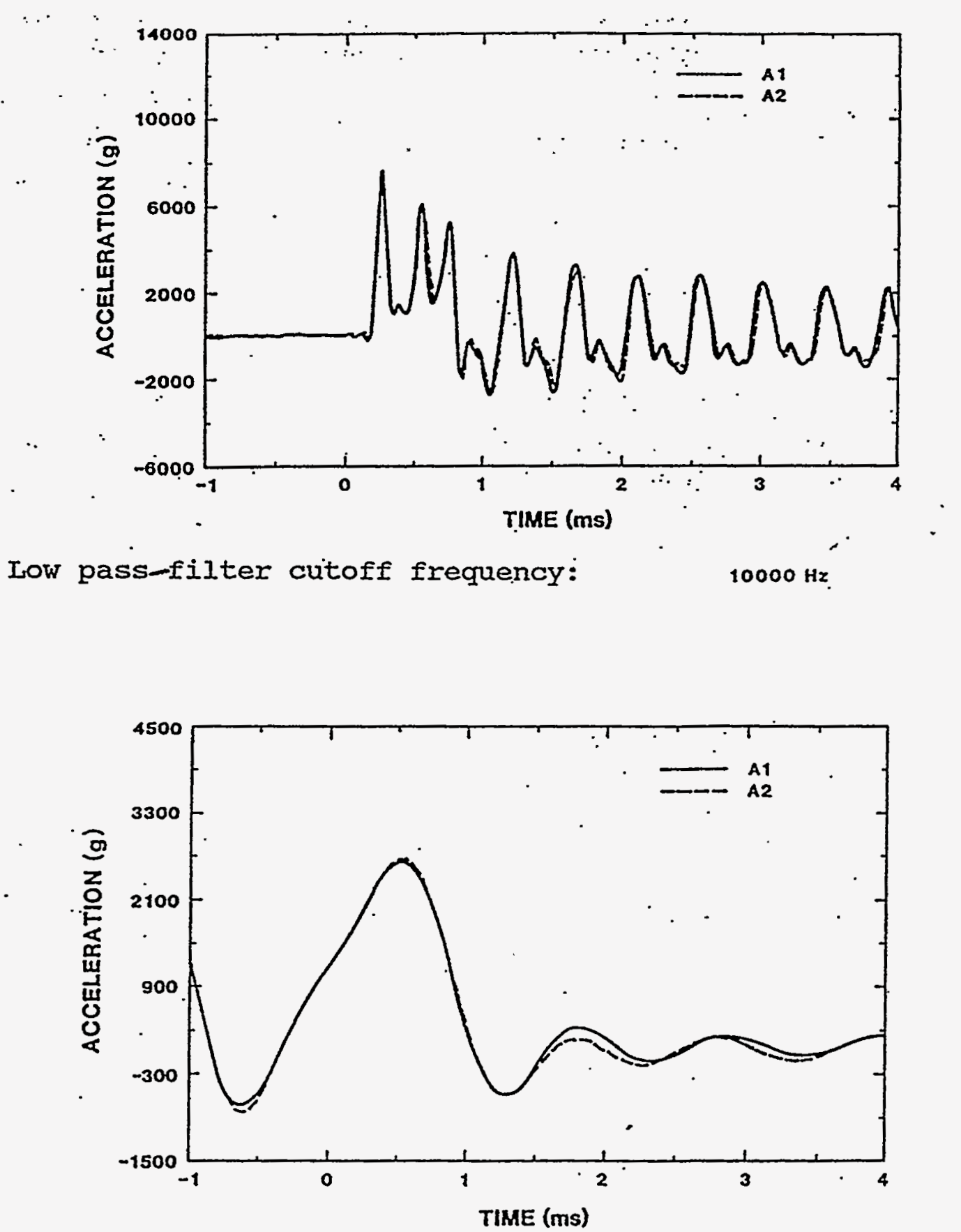

Low passwilter cutoff frequency:

$1000 \mathrm{~Hz}$

Figure C-10 Effect of Filter Cutoff Frequency on Acceleration Time History from Impact Test. 
Technical Information Department - Lawrence Livermore National Laboratory University of California - Livermore, California 94551

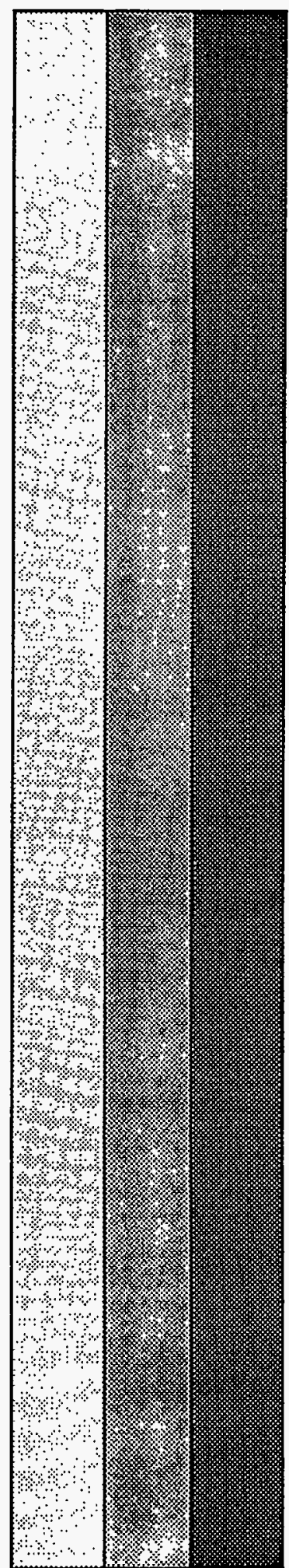

